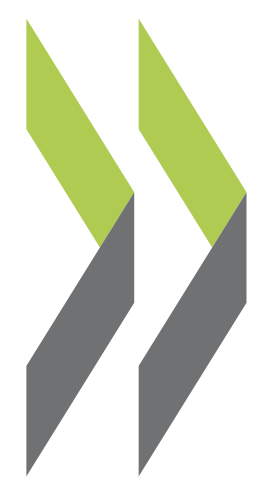

OECD Economics Department Working Papers No. 1268

\title{
The negative effect of regulatory divergence on foreign direct investment
} Jean-Marc Fournier 
Organisation de Coopération et de Développement Économiques

Organisation for Economic Co-operation and Development

13-Nov-2015

ECONOMICS DEPARTMENT

English - Or. English

\section{THE NEGATIVE EFFECT OF REGULATORY DIVERGENCE ON FOREIGN DIRECT INVESTMENT}

ECONOMICS DEPARTMENT WORKING PAPERS No. 1268

By Jean-Marc Fournier

OECD Working Papers should not be reported as representing the official views of the OECD or of its member countries. The opinions expressed and arguments employed are those of the author(s).

Authorised for publication by Jean-Luc Schneider, Deputy Director, Policy Studies Branch, Economics Department.

All Economics Department Working Papers are available at www.oecd.org/eco/workingpapers

JT03386239

Complete document available on OLIS in its original format

This document and any map included herein are without prejudice to the status of or sovereignty over any territory, to the delimitation of international frontiers and boundaries and to the name of any territory, city or area. 
OECD Working Papers should not be reported as representing the official views of the OECD or of its member countries. The opinions expressed and arguments employed are those of the author(s).

Working Papers describe preliminary results or research in progress by the author(s) and are published to stimulate discussion on a broad range of issues on which the OECD works.

Comments on Working Papers are welcomed, and may be sent to the Economics Department, OECD, 2 rue André-Pascal, 75775 Paris Cedex 16, France, or by e-mail to eco.contact@oecd.org.

All Economics Department Working Papers are available at www.oecd.org/eco/workingpapers.

The statistical data for Israel are supplied by and under the responsibility of the relevant Israeli authorities. The use of such data by the OECD is without prejudice to the status of the Golan Heights, East Jerusalem and Israeli settlements in the West Bank under the terms of international law.

\section{(C) OECD (2015)}

You can copy, download or print OECD content for your own use, and you can include excerpts from OECD publications, databases and multimedia products in your own documents, presentations, blogs, websites and teaching materials, provided that suitable acknowledgment of OECD as source and copyright owner is given. All requests for commercial use and translation rights should be submitted to rights@oecd.org 


\section{ABSTRACT/RÉSUMÉ \\ The negative effect of regulatory divergence on foreign direct investment}

The determinants of foreign direct investment (FDI) are explored with gravity models, using a Poisson estimator and a linear estimator, both with fixed effects. The heterogeneity of product market regulations has a large and robust impact on cross-border investment: a reduction of regulatory divergence by one fifth could increase FDI by about $15 \%$. In particular, the divergence of command and control regulations and of protection of incumbents (antitrust exemptions, entry barriers in networks and services) reduce cross-border investment. In addition, countries with higher employment protection have both less inward and less outward FDI, and there is some evidence that more complex regulatory procedures reduce inward FDI.

JEL classification codes: F15; F21; F23; K20

Keywords: Foreign direct investment, gravity models, product market regulation, heterogeneity, multinational firms

\section{L'effet négatif de l'hétérogénéité des réglementations sur l'investissement direct étranger}

Les déterminants de l'investissement direct étranger (IDE) sont analysés au moyen de modèles de gravité, en utilisant un estimateur de Poisson et un estimateur linéaire à effets fixes. L'hétérogénéité des réglementations des marchés de produits a un impact significatif et durable sur l'investissement international: une réduction d'un cinquième des différences entre les réglementations nationales pourrait accroître l'IDE d'environ $15 \%$. En particulier, les divergences entre les réglementations du type injonction et contrôle et entre les protections des acteurs en place (en termes de dérogations au droit de la concurrence, d'obstacles à l'entrée dans les industries de réseau et les secteurs de services) sont un frein à l'investissement international. En outre, les pays dotés d'une législation plus rigoureuse sur la protection de l'emploi enregistrent à la fois moins d'IDE entrant et moins d'IDE sortant, et des éléments montrent que des procédures réglementaires plus complexes réduisent l'IDE entrant.

Classification JEL : F15 ; F21 ; F23 ; K20

Mots clés : IDE, modèles de gravité, réglementation des marchés de produits, hétérogénéité, entreprises multinationales 


\section{TABLE OF CONTENTS}

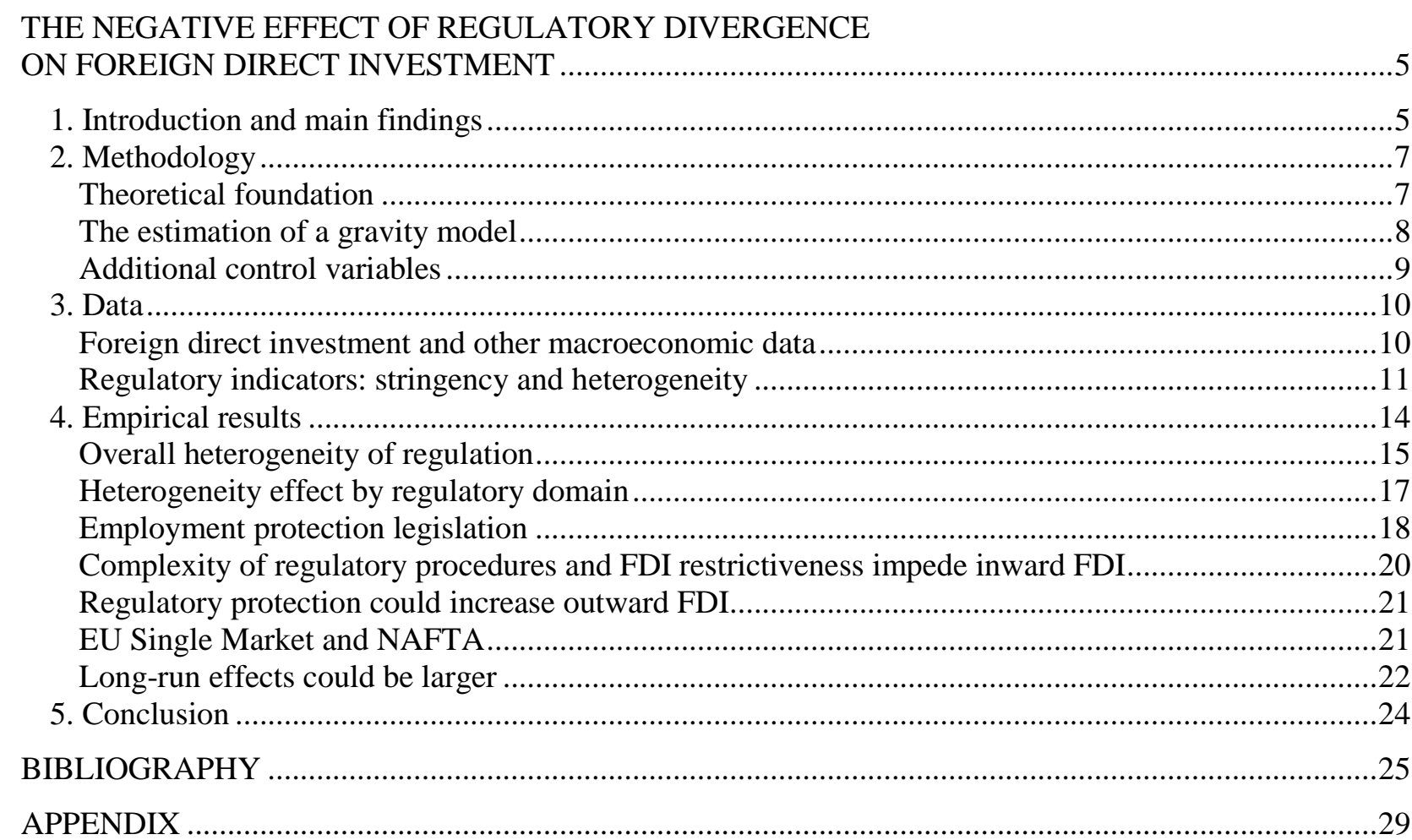

\section{Tables}

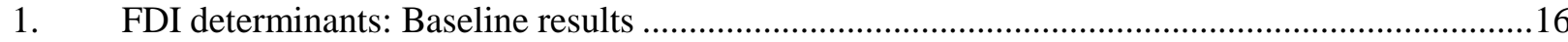

2. $\quad$ FDI determinants: Robustness check with time-varying fixed effects........................................17

3. The role of heterogeneity by selected low-level regulation domain ...........................................18

4. $\quad$ FDI determinants: Results with a 1990-2013 sample ................................................................19

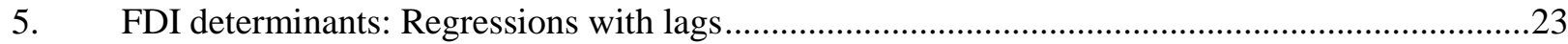

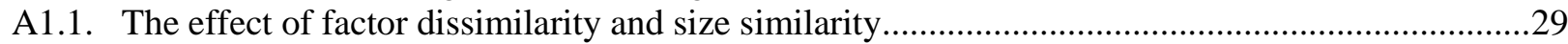

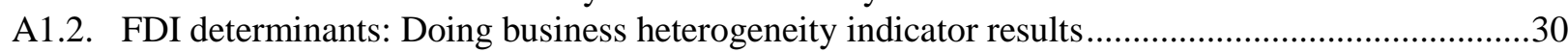

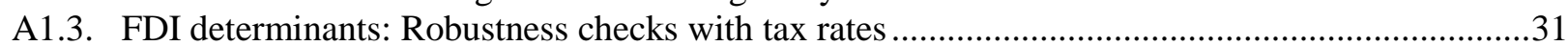

A1.4. FDI determinants: Excluding countries with large FDI flows ...................................................32

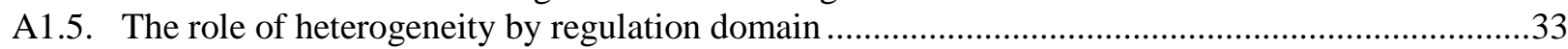

A1.6. The effect of stringency of product market regulation by sub-domain .......................................34

A1.7. FDI determinants: Detailed results with a 1990-2013 sample .................................................35

\section{Figures}

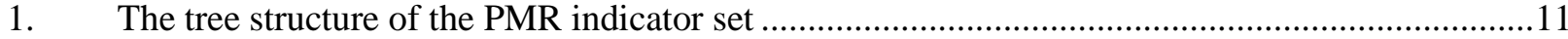

2. Product market regulation heterogeneity ................................................................................

3. Most countries that ease regulations move towards common practices.......................................14

4. Reforms of employment protection legislation can boost FDI .................................................20

5. The simplification of regulatory procedure can boost FDI .......................................................21 
ECO/WKP(2015)86

\title{
THE NEGATIVE EFFECT OF REGULATORY DIVERGENCE ON FOREIGN DIRECT INVESTMENT
}

\author{
By Jean-Marc Fournier ${ }^{1}$
}

\section{Introduction and main findings}

1. The beneficial effects of foreign direct investment (FDI) on efficiency and growth are well recognised and successive bilateral and multilateral investment agreements have reduced barriers to FDI. Beyond explicit barriers to FDI, national regulations can also have a significant impact on activities of multinational enterprises (MNEs). In particular, the differences in regulatory settings generate costs that can affect firms' decisions to invest abroad. Prior experience in a host country increases a firm's propensity to invest in that country relative to other options and inexperienced firms exhibit a greater preference for near, similar markets (Davidson, 1980), as firms pay an entry cost to get familiar with a different environment. However, the literature on the effect of regulation on the economy has essentially focused on the level of regulation (e.g. Blanchard and Giavazzi, 2003), and the indicators capture the stringency of regulations (e.g. Koske et al., 2015). The evidence on the role of regulatory heterogeneity is scarce. This paper investigates the empirical link between regulatory divergence and the FDI stock.

2. FDI can spur growth through numerous channels and this motivates policies aimed at reducing explicit or implicit barriers to FDI. First, FDI can promote the diffusion of ideas (Barrell and Pain, 1997). Aghion et al. (2006) argue that moving towards the technology frontier can be underpinned by foreign investment, if it involves the transfer of frontier technology. In addition, a knowledge technology transfer can spill over from foreign to domestic firms (Balasubramanyam et al., 1996; Javorcik, 2004). Second, the presence of MNEs can increase competition and hence provides an incentive for local firms to innovate. Third, the development of large MNEs can lead to economies of scale. This can rise in importance in the presence of knowledge-based capital: the initial cost (e.g. R\&D) do not arise again as the knowledge is combined with other inputs (Andrews and Criscuolo, 2013). Fourth, FDI can favour integration in global value chains. On top of the growth effect, FDI allows greater risk diversification (Rowland and Tesar, 2004).

3. Reaping the full benefits of FDI requires a policy setting that allows the reallocation of resources. Busse and Groizard (2008) find that FDI does not stimulate growth in economies with strict business and labour regulations. In addition, Alfaro et al. (2004) find that countries with well-developed financial markets gain significantly from FDI. FDI has to be underpinned by investment in human capital (Balasubramanyam et al., 1999). Last, Lesher and Miroudot (2008) show that FDI spillovers interact positively with trade liberalisation.

1. Jean-Marc Fournier is an economist in the Economics Department of the OECD. The author thanks Jens Arnold, Sónia Araújo, Christian Daude, Antoine Goujard, Asa Johansson, Céline Kauffmann, Peter Hoeller, Catherine Mann, Giuseppe Nicoletti, Hildegunn Nordås, Mauro Pisu, Jean-Luc Schneider, Cyril Schwellnus, Stéphane Sorbe, Jan Strasky, Petar Vujanovic, Eckhard Wurzel, Naomitsu Yashiro and participants of an internal OECD seminar for their relevant comments and suggestions, Debra Bloch, Emilie Kothe and Isabelle Wanner for their useful statistical support, and Celia Rutkoski for excellent editorial support. 
4. There is a considerable body of evidence on the effect of policies, including regulations, on FDI. In particular, Golub et al. (2003) find that FDI restrictions, employment protection legislation in the host country and exchange rate volatility have a negative impact on FDI. They also find a positive effect of nontariff trade barriers, suggesting that FDI may be a vehicle to bypass these barriers. Keller and Levinson (2002) find a moderate deterrent effect of pollution abatement costs on FDI in the United States. In addition, there is a negative correlation between the length of trials and FDI in EU countries (Fournier, 2014). The trial length can be a proxy for the legal uncertainties surrounding the security of property rights and the enforcement of contracts, which are important in shaping investment decisions (Palumbo et al., 2013). More broadly, the literature also provides evidence of the negative effect of regulations on business investment (e.g. Araújo, 2011 among others).

5. While there are numerous studies that investigate the distortive effect of the strictness of regulations on FDI, the evidence of the role of the heterogeneity of such regulations on FDI is scarce. In some studies, the level of the overall indicator of policy settings is replaced by their difference between the host and the investing country. Golub et al. (2003) find that the difference in the level of product market regulation between the host and the investing country and the difference in tax wedges affect FDI. However, these determinants are not proxies for the real divergence between regulatory settings: countries can have the same overall indicator with substantial differences that can be observed at a lower level only. Golub et al. (2003) thus interpret that it is a reduction of the level of regulation or taxation in the host countries that can increase FDI. In the same vein, Habib and Zurawicki (2002) find that an absolute difference in the prevalence of corruption between two countries reduces FDI.

6. This paper considers the bilateral differences between regulatory settings at a low level of aggregation to measure regulatory heterogeneity. This approach has been pioneered by Kox et al. (2004), Kox and Lejour (2006) and Nordås and Kox (2009), who found a large effect of heterogeneity on bilateral intra-EU trade and intra-EU FDI in services. However, these analyses were restricted to the heterogeneity of service regulations, and only one wave of product market regulation data was available for the first two studies. More recently, Fournier et al. (2015) find a large effect of regulatory heterogeneity on trade between OECD countries, and this paper is making use of a similar methodology to assess the effect of regulatory heterogeneity on FDI. With this approach, two countries with the same overall stringency level can be heterogeneous if the regulations are in different fields or if regulatory settings differ within fields.

7. This paper provides evidence that given a choice between a familiar and a less familiar regulatory environment, firms will prefer the former. The following main findings emerge from the empirical analysis:

- A reduction of regulatory stringency is associated with a reduction of heterogeneity.

- The divergence of product market regulation has a significant and robust negative effect on FDI. A broad reform package that would cut heterogeneity by one fifth could increase FDI by about 15\%. For instance, such a pace of convergence has been observed between 2008 and 2013 for pairs of countries such as Austria and the Slovak Republic, with both of them reducing countryspecific regulations.

- This divergence effect matters more in some fields of regulation, especially in the protection of incumbents. In particular, there is a negative and robust effect of divergence of antitrust exemptions and divergence of barriers in services. There is also a large negative effect that is significant in some, but not all, specifications for the heterogeneity of command and control regulations and in the heterogeneity of barriers in network sectors.

- The stringency of employment protection legislation can have a large negative impact on FDI. If all countries ease employment protection as it was done between 2003 and 2013 in those 15 OECD countries where the EPL index has decreased, FDI could increase by about $25 \%$. 
- The level of FDI restrictions has a significant negative impact on FDI.

- The complexity of regulatory procedures can have a negative impact on FDI. If countries that have complex regulatory procedures move towards the average of the top half of best performers, FDI could increase by about $15 \%$ according to some but not all specifications.

- There is some evidence that belonging to the EU Single Market can have a positive effect on FDI on top of its effect that is already captured by regulation indicators. By contrast, there is a negative effect for the North American Free Trade Agreement (NAFTA) area, which may reflect a substitution effect in favour of trade.

8. This paper is structured as follows. The second section presents the methodology, a gravity model estimated with a Poisson estimator as in Santos Silva and Teneyro (2006) and with a linear estimator. The third section describes the data. The fourth section discusses the empirical results for the different policy areas.

\section{Methodology}

\section{Theoretical foundation}

9. This paper uses a gravity model to estimate the effect of policies on FDI. In gravity models, bilateral FDI increases with the economic size of each partner, and decreases with geographical distance. This empirical approach is commonly used in the international trade literature, building on the theoretical foundations of Anderson and van Wincoop (2003). A growing empirical literature also uses the gravity equation to study the determinants of FDI (e.g. Eaton and Tamura, 1994; Bénassy-Quéré et al., 2007; Head and Ries, 2008), and of other issues such as cross-border portfolio investment flows (e.g. Portes and Rey, 2005).

10. Head and Ries (2008) provide a theoretical foundation for gravity models in which inward FDI stocks are determined by the characteristics of countries and a vector or pair-specific variables reflecting monitoring costs. This is a model of merger and acquisition decisions in which acquisitions go to the highest bidder, who has the highest anticipated return net of monitoring costs. There is thus a trade-off between the firm's ability to generate profits and proximity. In such a model, the expected bilateral FDI stock reflects structural parameters, and in case of a structural change, firms would undertake mergers and acquisitions to reach the new optimal FDI stock level. Their model shows that the expected FDI stock is increasing in origin and destination size variables and decreasing in measures of bilateral monitoring costs. As suggested by de Sousa and Lochard (2011), this approach can be extended to greenfield investments in which firms select the best projects across countries. Okawa and van Wincoop (2012) also provide a theoretical underpinning for the use of gravity models for international portfolio investments, a slightly different issue. In this context, they highlight that the inclusion of country fixed effects is necessary to produce unbiased estimates.

11. The heterogeneity of product market regulation generates monitoring and compliance costs. These monitoring costs are also captured by distance, contiguity, common legal system and common language variables. In addition, remoteness is expected to have, ceteris paribus, a positive effect on FDI: FDI between New Zealand and Australia, for instance, can be larger than FDI between two European countries separated by the same distance because of the lack of alternative partners. Remoteness is defined as the GDP-weighted average distance between a given country and its partners.

12. The literature also provides theories of how multinationals organise their production, providing additional insights about the relevant FDI determinants. In particular, in the knowledge-capital model developed by Markusen et al. (1996) and Markusen (1997), horizontal multinationals should be associated 
with similarities between countries in both size and relative factor endowments. If countries are dissimilar in size, the largest country can be favoured to avoid costly capacity in the smaller market. If countries are dissimilar in factor endowments, horizontal firms are more likely to concentrate if countries that where factors match their activity best. By contrast, vertical firms may locate different activities in each country. This model also suggests that the sales of affiliates would be a relevant dependent variable. Bilateral data on sales of affiliates are not systematically collected and in this respect, the FDI stock is a better proxy then FDI flows.

\section{The estimation of a gravity model}

13. The econometric analysis uses a Poisson model proposed by Santos Silva and Teneyro (2006) and applied for FDI stocks by Head and Ries (2008). Santos Silva and Teneyro (2006) showed that a linear estimation of the log-linearised gravity equation is valid only with a specific assumption on the distribution of the residuals. This specific assumption does not necessarily hold in practice. In particular, estimates can be biased in the presence of heteroskedasticity. In addition, the Poisson model makes it possible to take into account cases where the dependent variable is equal to zero. For these reasons, this paper uses the following Poisson model estimated with the Poisson pseudo-maximum likelihood (PPML) estimator:

$$
\begin{gathered}
F D I_{i j t}=\exp \left(\beta_{1} \ln \left(Y_{i t}\right)+\beta_{2} \ln \left(Y_{j t}\right)+\sum_{k} \beta_{3, k} d_{i j}^{k}+\beta_{4} \ln \left(R_{i t}\right)+\beta_{5} \ln \left(R_{j t}\right)+\sum_{k} \beta_{6, k} X_{i t}^{k}+\sum_{k} \beta_{7, k} X_{j t}^{k}+\right. \\
\left.\beta_{8} F T A_{i j t}+\beta_{9} F T A_{i t}+\beta_{10} F T A_{j t}+\beta_{11} E A_{i j t}+\gamma P M R_{i j t}^{h}+u_{i}+v_{j}+\alpha_{t}\right)+\varepsilon_{i j t}
\end{gathered}
$$

where $F D I_{i j t}$ denotes the nominal FDI inward foreign capital stock of country $i$ from country $j$ in year $t, Y_{i t}$ denotes the nominal GDP of country $i, d_{i j}^{k}$ refers to a set of four variables: the geographical distance, the contiguity, the existence of a common language and a dummy equal to one if the host and investing countries share the same legal system, ${ }^{2} R_{i t}$ denotes the remoteness defined as the GDP-weighted average distance between a given country and its partners, $X_{i t}^{k}$ is a set of country-specific policy variables, $F T A_{i t}$, $F T A_{j t}$ and $F T A_{i j t}$ refer to a combination of dummy variables to capture either the fact that a country belongs to a free trade area (European Economic Area, EEA; or North American Free Trade Agreement, NAFTA) or that a pair of countries belongs to the same free trade area and $E A_{i j t}$ is a dummy variable that takes value one when both countries are in the euro area. $P M R_{i j t}^{h}$ denotes the bilateral heterogeneity of the PMR indicator. $u_{i}, v_{j}$ and $\alpha_{t}$ denote host country, investing country and year fixed effects respectively. $\varepsilon_{i j t}$ denotes a zero-mean error. For symmetric indicators such as heterogeneity, the effect is the same on host and investing countries.

14. Country fixed effects reduce the risk of omitted variable bias and year fixed effects capture the global macroeconomic cycle. In the presence of country and year fixed effects, if a country has regulation that is persistently more restrictive than its peers, this does not drive the result. Similarly, if all countries make the same regulatory changes over time, this is does not drive the result either. Only different changes across countries contribute to the estimation. As regards bilateral measures, such as regulatory divergence or free trade areas, results are also driven by country pairs for which this bilateral indicator differs from the average value for each of the two countries relative to all counterparts. The standards errors are clustered by country-pair because there can be a high persistence of the level of the FDI stock within each countrypair over time.

15. To investigate the robustness of the results, this PPML estimator is replaced in some regressions by a linear estimator of the gravity model in which the dependent variable is the logarithm of FDI.

2. Stein and Daude (2007) also find that time zone differences affect FDI. However, it is not included here as most OECD countries are in the Northern hemisphere, so that geographical distance is well correlated with time zone difference. The time zone difference is indeed not significant with the sample used here. 
16. To check that there is no bias due to the omission of country-specific features that can vary over time, a robustness check is carried out with time-varying host country $\left(\mathrm{u}_{\mathrm{it}}\right)$ and investing country $\left(\mathrm{v}_{\mathrm{jt}}\right)$ dummies. Such time-varying fixed effects capture the effect of the stringency of regulation, of any type of country-specific policy setting that can change over time (e.g. taxation) and of other country-specific developments (e.g. exchange rate changes, local financial market developments).

$$
F D I_{i j t}=\exp \left(\sum_{k} \beta_{3, k} d_{i j}^{k}+\beta_{8} F T A_{i j t}+\beta_{11} E A_{i j t}+\gamma P M R_{i j t}^{h}+u_{i t}+v_{j t}\right)+\varepsilon_{i j t}
$$

17. In gravity models with controls for GDP, the effect of a policy change on FDI that goes through a change of GDP is not captured by policy coefficients. With a GDP elasticity close to one, the coefficients show the effects of FDI determinants on FDI intensity as defined by the FDI/GDP ratio, rather than effects on FDI levels. In particular, a policy that has a similar impact on GDP and on FDI, and hence has no impact on FDI intensity, is associated with a null coefficient. In addition, such gravity models do not allow to disentangle to what extent FDI decisions are driven by absolute levels of the policy stance (so that if all countries change in the same direction, the FDI intensity changes) or by the levels of the policy stance relative to country peers (so that if all countries change in the same direction, relative policy settings and hence the FDI intensity would not change). Illustrative magnitudes of FDI effects presented in this paper are calculated under the assumption that results are driven by absolute levels of the policy stance.

\section{Additional control variables}

18. As discussed in Ethier and Markusen (1996), differences in factor endowments can affect FDI. The optimal factor structure depends on the structure of multinational firms, especially whether they are vertically integrated and may need different production factors at different stages of production, or horizontally integrated and hence need similar production factors. As a result, the effect of factor dissimilarity is ambiguous. The findings of Ethier and Markusen (1996) suggest that the second model plays a larger role than the first one. Differences in endowments are proxied by factor dissimilarity $(F D)$ and human capital dissimilarity $(H C D)$, as in Golub et al. (2003):

$$
\begin{aligned}
& F D_{i j t}=\left|\ln \left(\frac{Y_{j t}}{\text { pop }_{j t}}\right)-\ln \left(\frac{Y_{i t}}{\text { pop }_{i t}}\right)\right| \\
& H C D_{i j t}=\left|\ln \left(e d u c_{j}\right)-\ln \left(e d u c_{i}\right)\right|
\end{aligned}
$$

where the dissimilarity in GDP per capita is regarded as a proxy for the dissimilarity in the capital stock per worker and the education variable (educ) refers to the average number of years of schooling.

19. In the same vein, an investor may find it easier to invest in a country with a similar size that could present more similarities with its home country. Following Golub et al. (2003), size similarity can thus also be added as a control variable:

$$
S S_{i j t}=\ln \left(1-\left(\frac{Y_{i t}}{Y_{i t}+Y_{j t}}\right)^{2}-\left(\frac{Y_{j t}}{Y_{i t}+Y_{j t}}\right)^{2}\right)
$$

20. The size of the FDI stock can also be modified by valuation changes. The nominal exchange rate in host and investing countries are added as control variables to capture a part of these changes. These variables can also capture a part of competitiveness changes. One should not interpret the coefficients associated with the exchange rate as a causal effect as the causality runs both ways: capital flows can have an impact on the exchange rate. In practice, as there is no particular link between exchange rate changes and the policy determinants considered here, the inclusion of these control variables does not modify the results of interest much. In addition, the variation of GDP itself captures a part of the competitiveness 
changes. ${ }^{3}$ A dummy that takes value one when both countries belong to the euro area is also added to capture the effect of the absence of currency risks.

\section{Data}

\section{Foreign direct investment and other macroeconomic data}

21. This paper is making use of inward FDI stocks in value (US dollars) reported in OECD FDI databases. FDI is the category of international investment that reflects the objective of a resident entity to obtain a lasting interest in an enterprise resident in another country. The direct or indirect ownership of $10 \%$ or more of the voting power is considered as evidence of the existence of such a lasting interest: small participations that are typically not associated with a significant influence are not included. These FDI positions can be the result of greenfield investments or of mergers and acquisitions, the latter amounting to about two thirds of global FDI flows. The FDI stock is net: it is measured as the market value share capital and reserves plus debt due to direct investors minus debt due to subsidiaries. As a result, FDI bilateral stocks can be negative; this is the case for about $5 \%$ of bilateral stocks that are dropped here. ${ }^{4}$ The FDI bilateral data also exhibit slightly more than $10 \%$ of zero stocks that are kept in the sample. In the loglinear alternative method the log of FDI is replaced by zero in these cases.

22. The OECD FDI data published in March 2015 have been compiled using the fourth edition of the OECD Benchmark Definition of FDI (OECD, 2008), and previous vintages had been compiled according to the third edition (OECD, 1996). Countries mostly reported 2013 data only according to this new standard (a few countries also reported data from 2009 to 2012), while FDI stocks between 1990 and 2012 (and in 2013 for a few countries) are available according to the third edition of standards.

23. In most empirical estimates of this paper, the third edition FDI data are pooled with the fourth edition data, selecting a measure of this new vintage that is as close as possible to the one used in the third edition. In the fourth edition, the counterpart can be the immediate investor or the ultimate investor, and the stock can include Special Purpose Entities (SPEs) or not. The first option is chosen for both cases to better match with the concept used in the third edition. In addition, the sample of bilateral FDI stocks for which data according to the ultimate investor concept are available is fairly limited. In practice, for the 441 overlapping observations, the correlation between the third and the fourth edition data is 0.99 , with almost the same means. It is thus a reasonable approximation to pool the third and fourth edition data, ignoring the slight differences due to definition changes. In this pooled approach, when both editions are available, the fourth one is considered.

24. The baseline country sample used in this analysis are OECD countries in which the share of FDI assets owned by SPEs does not exceed $80 \%$ according to the fourth edition data (Hungary, Luxembourg and the Netherlands are thus excluded). SPEs have little or no physical presence in the country and hence are less likely to be affected by regulations of the country and the related bilateral heterogeneity, except for some specific aspects, such as antitrust exemptions or the inclusion in the Single Market.

3. The inclusion of the real effective exchange rate has also been investigated. It does not alter the results of interest much. By contrast, it alters the coefficients associated with GDP, as GDP changes can be correlated with competitiveness changes.

4. The issue of negative data is an additional argument to use FDI stocks, rather than flows. FDI flow data can exhibit numerous negative flows that are not straightforward to interpret: a large negative flow could reflect a deterioration of business conditions, but also be the existence of a few large deals in a pair of countries with tight links that go both directions. 
25. The OECD Benchmark Definition recommends market value as the conceptual basis for valuation where possible (OECD, 1996 and OECD, 2008). However, in practice enterprises' balance sheet values represent the only source readily available in most countries. They may be reported on a current market value basis, but also on a historical cost basis, or based on some interim but not current market value.

26. The other macroeconomic variables are provided by the OECD Analytical Data Base (ADB). The average distance between the main cities of each country pair weighted by population, as well as language and contiguity data, are taken from CEPII's GeoDist database. The average number of years of schooling are those by Barro and Lee (2013). The legal system classification used here is the same as in Djankov et al. (2007), grouping countries into four legal origins: the Common law, the French law, the German law and the Nordic law. For the alternative regressions with tax rates, the average effective tax rates are from the Oxford University Centre for Business Taxation database, and the average tax wedges at the average earnings level for a single person and for one-earner married couples with two children are from the OECD Taxing Wages Database.

\section{Regulatory indicators: stringency and heterogeneity}

27. The product market regulation indicator (PMR) is an economy-wide indicator measured every five years between 1998 and 2013 that covers a wide range of goods and service sectors. The PMR database includes more than 700 questions, which are aggregated to three high-level components: state control, barriers to entrepreneurship and barriers to foreign trade and investment (Figure 1 and Koske et al., 2015).

Figure 1. The tree structure of the PMR indicator set

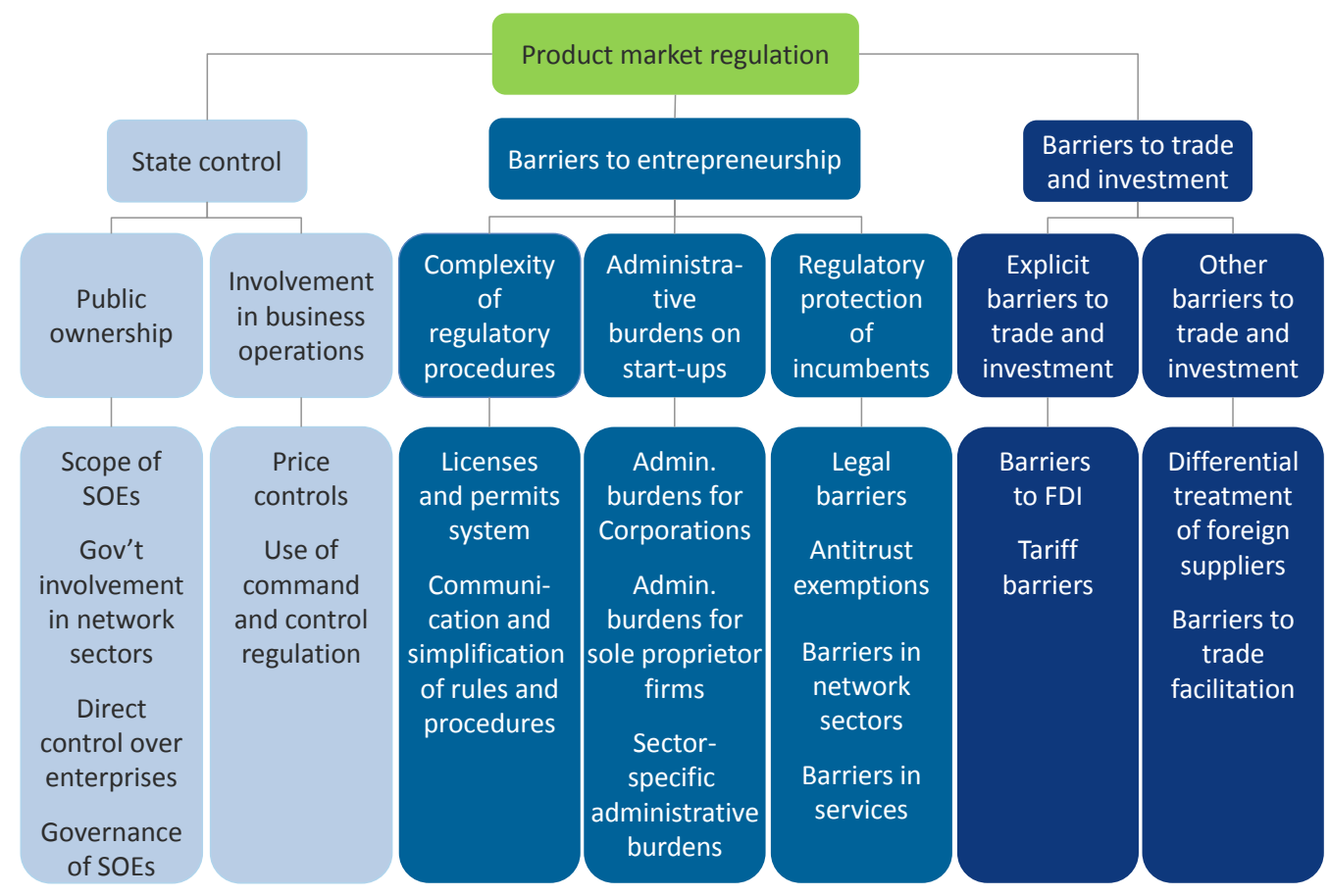

Source: Koske, I. et al. (2015), "The 2013 Up-date of the OECD Product Market Regulation Indicators: Policy Insights for OECD and non-OECD Countries", OECD Economics Department Working Paper, No. 1200, OECD Publishing. 
28. Among barriers to trade and investment, the barriers to FDI sub-component is measured by the FDI restrictiveness index (Kalinova et al., 2010). This captures restrictiveness of a country's FDI rules in 22 sectors in terms of foreign equity limitations, screening or approval mechanisms, restrictions on the employment of foreigners as key personnel and operational restrictions (e.g. restrictions on branching and on capital repatriation or on land ownership).

29. The bilateral heterogeneity of regulation is measured as the share of regulatory settings that differ between two countries, making use of the OECD PMR indicator (Fournier, 2015) or the World Bank Doing Business indicator between 2003 and 2007 (Nordås and Kox, 2009). The methodology is similar to the one used by Kox et al. (2004) for service regulations. For each question considered in the questionnaire, a country pair is assigned the value 1 if the answer is different and 0 otherwise. Almost all questions are qualitative, with a limited set of possible answers. These results are averaged using the same weights as in the overall economy PMR indicator. For a given country, its heterogeneity with respect to each other OECD country can be calculated, and an average of each of these bilateral measures provides insight into how much a country differs from other OECD countries. The resulting economy-wide measure is a lower bound of regulatory heterogeneity as even when the answer is the same, regulation can differ for some aspects that are not captured in this questionnaire (e.g. a different implementation of the same regulation).

30. The investigation of product market regulation heterogeneity at the question level provides more information than simply looking at the difference of the PMR indicators: the correlation between the absolute differences between overall PMR indicators and the heterogeneity indicator is 0.4 . This opens the way for regressions including both PMR stringency and PMR heterogeneity so as to disentangle the two effects.

31. Some questions required specific adjustments. Two sub-components of the state control component, namely the scope of state-owned enterprises and direct control over business enterprises (e.g. existence of government golden shares), do not cover regulations for which heterogeneity directly affects cross-border activities of firms. These sub-components are thus excluded from the calculation of bilateral regulatory heterogeneity. For the questions related to the adoption of international standards and Mutual Recognition Agreements, two countries are regarded as similar only if both provide a positive answer. Some quantitative variables are replaced by dummies: typical costs in local currency to register a business are replaced by a dummy variable that captures whether there is a cost or not, and questions on shop opening hours are replaced by a dummy variable capturing whether shop opening hours are regulated or not. ${ }^{5}$

32. The Czech Republic, the Netherlands and Switzerland are the closest to common practices, while Israel, Mexico and Turkey are the furthest away (Figure 2). To a large extent, this reflects the level of overall regulatory stringency as measured by the headline PMR indicator: the correlation across countries between the stringency of regulation and the average bilateral heterogeneity was 0.67 in 2013 . The tighter regulatory restrictions are, the more they generate divergences with other countries. This is because for many questions covered by the PMR questionnaire, less stringent stances are more common across OECD countries. There are exceptions such as Austria and the United Kingdom that are not particularly close to other countries in terms of regulatory settings even though regulatory stringency is low. Looking into regulatory heterogeneity by seven sub-domains, the share of different bilateral settings is the smallest in

5. Compared to a naïve approach without question-specific changes, these data adjustments slightly increase the estimates of the adverse effect of heterogeneity on FDI: measurement errors generate an attenuation bias of the effect of heterogeneity. 
the domain "other barriers to trade and investment", which is also a domain with a quite low average stringency level. By contrast, it is the highest in the domain "administrative burdens on start-ups". 6

Figure 2. Product market regulation heterogeneity

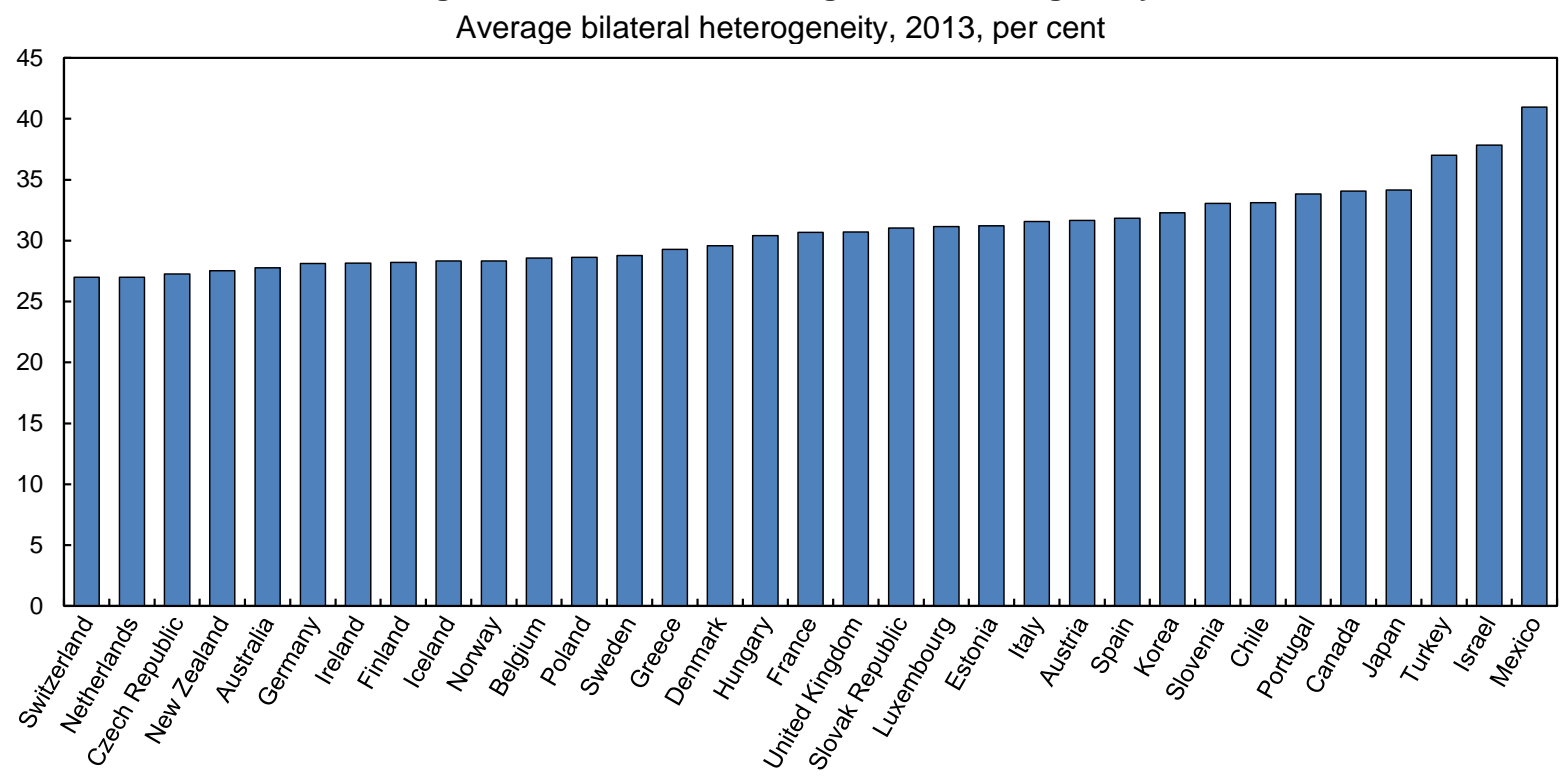

Note: The United States PMR data are available until 2008 only, and hence the United States is not shown in this figure. Source: OECD, Product Market Regulation database and OECD calculations.

33. The effect of each country's reforms on heterogeneity can be assessed by comparing the actual average heterogeneity of a given country to the average heterogeneity of this country if it had kept regulations unchanged between 2008 and 2013, while other countries were reforming. 21 out of 29 countries that eased regulatory settings on average since 2008 have also moved towards common practices (Figure 3). In addition, countries that were the furthest away from common practices reduced heterogeneity most (Fournier, 2015).

34. This analysis is also making use of an OECD measure of the strictness of employment protection legislation (EPL) for regular contracts. The first version of this indicator is used here because it is available from 1985 to 2013 and hence provides more variability over time than the second (1998-2013) or the third (2008-2013) version. ${ }^{7}$

6. Bilateral heterogeneity tables for EU countries that are members of the OECD are available in Fournier (2015).

7. In the first version, the indicator for regular contracts does not include the maximum time to make a claim of unfair dismissal and the additional provision for collective dismissals. 


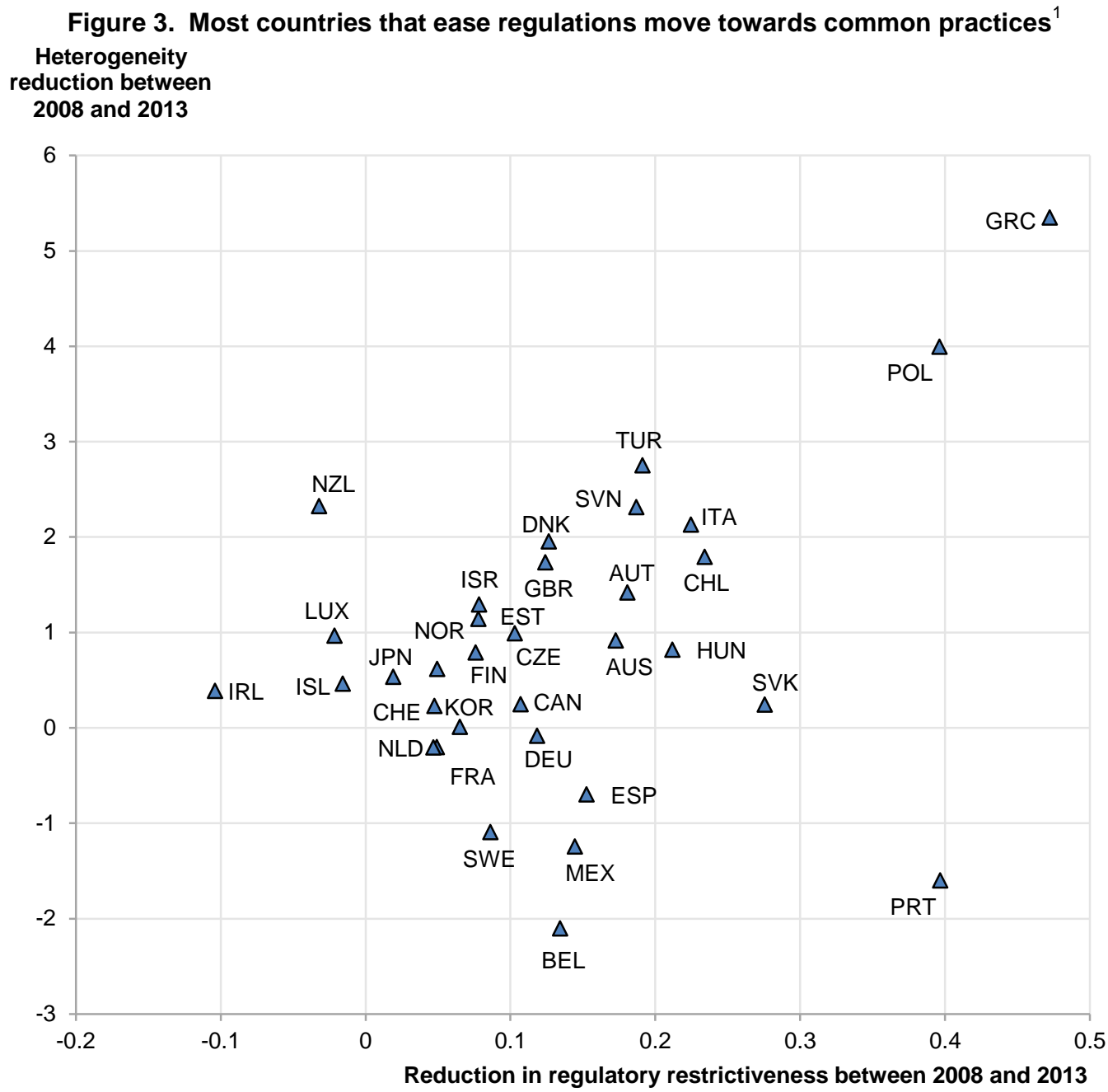

1. The vertical axis shows the difference between the average bilateral heterogeneity of a given country in 2013 and the average bilateral heterogeneity that would be observed if no change had been made in this country's regulations between 2008 and 2013.

2. The United States PMR data are available until 2008 only, and hence the United States is not shown in this figure.

Source: OECD, Product Market Regulation database and OECD calculations.

\section{Empirical results}

35. Various sets of regressions are used to investigate the role of policies and check the robustness of results. First, the effect of the heterogeneity of regulations on FDI is investigated in a baseline regression that makes use of the overall bilateral PMR heterogeneity, various additional policy variables and FDI data from 1998 to 2013, in which the third and fourth BMD data are pooled (Table 1). Second, a set of regressions with time-varying fixed effects shows that the results are robust to the control for any kind of country-specific characteristics that can vary over time (Table 2). Third, regressions with sub-domains of the PMR heterogeneity indicator shine a light on the areas in which regulatory divergence matters most (Table 3). Fourth, regressions with longer time series (1990-2013) are considered to better capture the effect of employment protection legislation and of the EU Single Market in particular (Table 4). Fifth, regressions with lagged determinants show that the long-run effects could be substantially larger (Table 5). Additional robustness checks in the annex show that the results hold with the Doing Business heterogeneity indicator from 2003 to 2007 , or if one controls for tax rates. 
36. As expected, regressions show that FDI increases with the size of the economy, the existence of a common language, a similar legal system or a common border and remoteness, and decreases with distance. The presentation of the results focuses here on the effect of policies that are discussed one by one.

\section{Overall heterogeneity of regulation}

37. The negative effect of regulatory divergence on FDI is sizeable (Table 1). Most specifications suggest that a reduction of FDI divergence by one fifth could increase FDI by about $15 \%$. For instance, such a pace of convergence has been observed between 2008 and 2013 for pairs of countries such as Austria and the Slovak Republic. This fast convergence pace is the result of easing regulations in both Austria and the Slovak Republic and in various fields at the same time. Beyond this illustrative effect for an average OECD country, the gains could be larger for countries that have more room for improvement. For instance, Mexico is the furthest away from the regulatory settings of other OECD countries (Figure 2). In this country, aligning its average bilateral heterogeneity to the OECD average could increase FDI by about $25 \%$.

38. While there is uncertainty surrounding the exact magnitude of this effect, as some specifications suggest an impact of a one-fifth reduction of FDI divergence ranging from about $4 \%$ to about $20 \%$, all estimates point to a clearly negative effect of regulatory divergence. In addition, the true effect could be even larger: as the heterogeneity measure built on the PMR indicator does not capture the design of regulations, it can be regarded as a proxy for the genuine heterogeneity with some measurement error, leading to an underestimation bias.

39. Tables 1, 2, 3, 5, A1.2, A1.3, A1.4 and A1.5 show the heterogeneity effect is particularly robust. The effect hardly changes when adding the stringency of product market regulation itself as a control variable (Table 1, Column 2, 3, 6 and 7). In alternative specifications, additional control variables capturing various forms of heterogeneity or similarity that can affect FDI on top of regulatory heterogeneity are added, and the negative effect of heterogeneity still holds (Table 1, Column 4 and 9). When one includes those countries with a high share of special purpose entities, the effect also holds (Table 1, Column 5 and 10), and the link between FDI and host country GDP weakens. Similar specifications are estimated with a Poisson and with a linear model, and the magnitude of the heterogeneity effect hardly depends on the type of estimator. Table A1.2 shows that the effect of heterogeneity as measured with the Word Bank's Doing Business database is also significantly negative and robust to changes in the list of control variables and to different estimation methods.

40. FDI stocks can also be driven by taxation (see OECD, 2007, for a detailed investigation), and this is independent of regulation: the effects of regulation on FDI are quite similar with the inclusion of controls for average corporate taxes and the labour tax wedge (Table A1.3 in the appendix). Table A1.4 also shows that if one excludes the five countries where FDI flows relative to GDP are the highest (Belgium, Ireland, Luxembourg, the Netherlands and Switzerland), results still hold.

41. On top of regulatory heterogeneity, other forms of heterogeneity matter as well. In particular, there is about $50 \%$ to $80 \%$ more FDI between countries that share the same legal system: this crucially simplifies monitoring. When one drops the legal system dummy, the adverse effect of product market regulation becomes larger. This suggests that there are deeply rooted forms of regulatory heterogeneity that are either captured with the PMR heterogeneity indicator or by the legal system dummy: in the regression with the legal system dummy, these are rather less deeply rooted forms of heterogeneity that drive the PMR heterogeneity effect. There is also robust evidence that firms prefer cross-border investment in countries of similar size, in line with the knowledge-capital model developed by Markusen (1997) (Tables 2 and A1.1). Last, there is some evidence that FDI can be lower if production factor endowments are more dissimilar, in line with Ethier and Markusen (1996). 
$\mathrm{ECO} / \mathrm{WKP}(2015) 86$

Table 1. FDI determinants: Baseline results

\begin{tabular}{|c|c|c|c|c|c|c|c|c|c|c|}
\hline Estimation method & $\begin{array}{c}(1) \\
\text { Poisson }\end{array}$ & $\begin{array}{c}(2) \\
\text { Poisson }\end{array}$ & $\begin{array}{c}\text { (3) } \\
\text { Poisson }\end{array}$ & $\begin{array}{c}\text { (4) } \\
\text { Poisson }\end{array}$ & $\begin{array}{c}\text { (5) } \\
\text { Poisson }\end{array}$ & $\begin{array}{c}(6) \\
\text { Linear }\end{array}$ & $\begin{array}{c}(7) \\
\text { Linear }\end{array}$ & $\begin{array}{c}(8) \\
\text { Linear }\end{array}$ & $\begin{array}{c}(9) \\
\text { Linear }\end{array}$ & $\begin{array}{l}(10) \\
\text { Linear }\end{array}$ \\
\hline $\ln \left(Y_{i t}\right)$ & $\begin{array}{c}1.21^{* * *} \\
(0.30)\end{array}$ & $\begin{array}{c}1.34^{* \star *} \\
(0.26)\end{array}$ & $\begin{array}{c}1.36^{\star \star \star} \\
(0.32)\end{array}$ & $\begin{array}{l}0.52^{*} \\
(0.31)\end{array}$ & $\begin{array}{l}0.42^{*} \\
(0.25)\end{array}$ & $\begin{array}{l}0.92^{* * *} \\
(0.34)\end{array}$ & $\begin{array}{c}0.86^{* * *} \\
(0.30)\end{array}$ & $\begin{array}{l}1.31^{* \star *} \\
(0.38)\end{array}$ & $\begin{array}{l}0.90^{* *} \\
(0.35)\end{array}$ & $\begin{array}{l}0.61^{* *} \\
(0.30)\end{array}$ \\
\hline $\ln \left(Y_{j t}\right)$ & $\begin{array}{c}1.36^{* * *} \\
(0.30)\end{array}$ & $\begin{array}{c}1.20^{\star \star \star} \\
(0.29)\end{array}$ & $\begin{array}{l}1.00^{* \star *} \\
(0.35)\end{array}$ & $\begin{array}{c}0.68^{\star * *} \\
(0.24)\end{array}$ & $\begin{array}{l}1.43^{* \star *} \\
(0.45)\end{array}$ & $\begin{array}{l}1.16^{\star \star *} \\
(0.30)\end{array}$ & $\begin{array}{c}1.29^{* * *} \\
(0.31)\end{array}$ & $\begin{array}{l}1.09^{* \star *} \\
(0.38)\end{array}$ & $\begin{array}{l}1.03^{\star \star \star} \\
(0.31)\end{array}$ & $\begin{array}{c}1.03^{\star \star *} \\
(0.28)\end{array}$ \\
\hline $\ln \left(\right.$ dist $\left._{i j}\right)$ & $\begin{array}{c}-0.64^{* * *} \\
(0.11)\end{array}$ & $\begin{array}{c}-0.64^{* * *} \\
(0.11)\end{array}$ & $\begin{array}{c}-0.64^{* * *} \\
(0.11)\end{array}$ & $\begin{array}{c}-0.58^{* * *} \\
(0.096)\end{array}$ & $\begin{array}{c}-0.60^{\star * *} \\
(0.085)\end{array}$ & $\begin{array}{c}-1.15^{\star * *} \\
(0.11)\end{array}$ & $\begin{array}{c}-1.15^{\star * *} \\
(0.11)\end{array}$ & $\begin{array}{c}-1.15^{\star * *} \\
(0.11)\end{array}$ & $\begin{array}{c}-1.17^{* * *} \\
(0.11)\end{array}$ & $\begin{array}{c}-1.03^{* * *} \\
(0.10)\end{array}$ \\
\hline Contiguity $_{\mathrm{ij}}$ & $\begin{array}{c}0.15 \\
(0.16)\end{array}$ & $\begin{array}{c}0.15 \\
(0.16)\end{array}$ & $\begin{array}{c}0.15 \\
(0.15)\end{array}$ & $\begin{array}{c}0.20 \\
(0.13)\end{array}$ & $\begin{array}{c}0.10 \\
(0.14)\end{array}$ & $\begin{array}{l}-0.16 \\
(0.23)\end{array}$ & $\begin{array}{l}-0.16 \\
(0.23)\end{array}$ & $\begin{array}{l}-0.16 \\
(0.23)\end{array}$ & $\begin{array}{l}-0.17 \\
(0.23)\end{array}$ & $\begin{array}{c}0.0060 \\
(0.21)\end{array}$ \\
\hline Same legal system ${ }_{i j}$ & $\begin{array}{l}0.46^{* * *} \\
(0.097)\end{array}$ & $\begin{array}{l}0.46^{\star * *} \\
(0.099)\end{array}$ & $\begin{array}{l}0.46^{* \star \star} \\
(0.099)\end{array}$ & $\begin{array}{l}0.44^{\star * *} \\
(0.088)\end{array}$ & $\begin{array}{l}0.34^{\star \star \star} \\
(0.084)\end{array}$ & $\begin{array}{c}0.61^{* * *} \\
(0.12)\end{array}$ & $\begin{array}{c}0.61^{* * *} \\
(0.12)\end{array}$ & $\begin{array}{l}0.61^{* * *} \\
(0.12)\end{array}$ & $\begin{array}{c}0.63^{\star * *} \\
(0.12)\end{array}$ & $\begin{array}{c}0.66^{* \star \star} \\
(0.10)\end{array}$ \\
\hline Common language $_{i j}$ & $\begin{array}{c}0.10 \\
(0.15)\end{array}$ & $\begin{array}{c}0.10 \\
(0.15)\end{array}$ & $\begin{array}{c}0.12 \\
(0.15)\end{array}$ & $\begin{array}{c}0.17 \\
(0.13)\end{array}$ & $\begin{array}{l}0.014 \\
(0.12)\end{array}$ & $\begin{array}{c}0.59^{* * *} \\
(0.21)\end{array}$ & $\begin{array}{c}0.59^{\star \star \star *} \\
(0.21)\end{array}$ & $\begin{array}{c}0.59^{* * *} \\
(0.21)\end{array}$ & $\begin{array}{c}0.69^{\star \star \star *} \\
(0.22)\end{array}$ & $\begin{array}{l}0.48^{* *} \\
(0.20)\end{array}$ \\
\hline $\ln \left(\right.$ Remoteness $\left._{\text {it }}\right)$ & $\begin{array}{l}-0.51 \\
(1.16)\end{array}$ & $\begin{array}{c}0.35 \\
(1.10)\end{array}$ & $\begin{array}{l}-0.61 \\
(1.25)\end{array}$ & $\begin{array}{l}-1.36 \\
(1.09)\end{array}$ & $\begin{array}{l}2.29^{*} \\
(1.21)\end{array}$ & $\begin{array}{c}2.00 \\
(1.91)\end{array}$ & $\begin{array}{c}1.36 \\
(1.94)\end{array}$ & $\begin{array}{c}1.86 \\
(1.99)\end{array}$ & $\begin{array}{c}1.79 \\
(1.99)\end{array}$ & $\begin{array}{c}2.26 \\
(1.76)\end{array}$ \\
\hline $\ln \left(\right.$ Remoteness $\left._{\mathrm{jt}}\right)$ & $\begin{array}{l}-1.81 \\
(1.15)\end{array}$ & $\begin{array}{l}-2.29^{*} \\
(1.17)\end{array}$ & $\begin{array}{l}-2.73^{\star *} \\
(1.33)\end{array}$ & $\begin{array}{c}-2.70^{\star *} \\
(1.10)\end{array}$ & $\begin{array}{l}-1.28 \\
(1.74)\end{array}$ & $\begin{array}{l}-1.06 \\
(1.93)\end{array}$ & $\begin{array}{l}-0.39 \\
(1.87)\end{array}$ & $\begin{array}{c}0.32 \\
(2.03)\end{array}$ & $\begin{array}{l}-1.40 \\
(1.98)\end{array}$ & $\begin{array}{l}-1.94 \\
(1.80)\end{array}$ \\
\hline 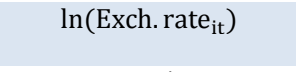 & $\begin{array}{l}-0.62 \\
(0.39)\end{array}$ & $\begin{array}{c}-0.87^{\star *} \\
(0.39)\end{array}$ & $\begin{array}{l}-0.73^{*} \\
(0.41)\end{array}$ & $\begin{array}{l}-0.032 \\
(0.41)\end{array}$ & $\begin{array}{l}-0.058 \\
(0.34)\end{array}$ & $\begin{array}{l}-0.94^{* *} \\
(0.47)\end{array}$ & $\begin{array}{l}-0.90^{*} \\
(0.47)\end{array}$ & $\begin{array}{c}-0.99^{* *} \\
(0.47)\end{array}$ & $\begin{array}{l}-0.65 \\
(0.51)\end{array}$ & $\begin{array}{l}-0.70^{*} \\
(0.40)\end{array}$ \\
\hline $\ln \left(\right.$ Exch. rate $\left._{j \mathrm{t}}\right)$ & $\begin{array}{c}-0.89^{* *} \\
(0.44)\end{array}$ & $\begin{array}{l}-0.48 \\
(0.41)\end{array}$ & $\begin{array}{l}-0.43 \\
(0.47)\end{array}$ & $\begin{array}{l}-0.32 \\
(0.47)\end{array}$ & $\begin{array}{l}-0.92 \\
(0.73)\end{array}$ & $\begin{array}{c}0.16 \\
(0.34)\end{array}$ & $\begin{array}{l}0.017 \\
(0.34)\end{array}$ & $\begin{array}{c}0.31 \\
(0.38)\end{array}$ & $\begin{array}{c}0.15 \\
(0.35)\end{array}$ & $\begin{array}{l}0.096 \\
(0.29)\end{array}$ \\
\hline $\mathrm{PMR}_{i t}$ & & $\begin{array}{l}-0.24 \\
(0.18)\end{array}$ & & & & & $\begin{array}{c}0.35 \\
(0.26)\end{array}$ & & & \\
\hline $\mathrm{PMR}_{j t}$ & & $\begin{array}{l}0.082 \\
(0.18)\end{array}$ & & & & & $\begin{array}{l}-0.28 \\
(0.25)\end{array}$ & & & \\
\hline $\mathrm{PMR}_{i j t}^{h}$ & $\begin{array}{c}-1.59^{* * *} \\
(0.61)\end{array}$ & $\begin{array}{c}-1.58^{* *} \\
(0.64)\end{array}$ & $\begin{array}{c}-1.45^{\star *} \\
(0.73)\end{array}$ & $\begin{array}{l}-0.61 \\
(0.55)\end{array}$ & $\begin{array}{c}-2.52^{* * *} \\
(0.64)\end{array}$ & $\begin{array}{c}-2.83^{* * *} \\
(0.83)\end{array}$ & $\begin{array}{c}-2.77^{* * *} \\
(0.84)\end{array}$ & $\begin{array}{c}-2.96^{* * *} \\
(0.86)\end{array}$ & $\begin{array}{c}-2.53^{\star * *} \\
(0.84)\end{array}$ & $\begin{array}{c}-2.40^{* * *} \\
(0.76)\end{array}$ \\
\hline $\mathrm{CRP}_{\text {it }}$ & $\begin{array}{c}-0.15^{\star \star *} \\
(0.050)\end{array}$ & & $\begin{array}{l}-0.16^{\star \star *} \\
(0.046)\end{array}$ & $\begin{array}{c}-0.17^{* * *} \\
(0.045)\end{array}$ & $\begin{array}{l}-0.14^{\star *} \\
(0.054)\end{array}$ & $\begin{array}{c}0.025 \\
(0.071)\end{array}$ & & $\begin{array}{c}0.013 \\
(0.070)\end{array}$ & $\begin{array}{c}-0.0016 \\
(0.069)\end{array}$ & $\begin{array}{l}-0.010 \\
(0.061)\end{array}$ \\
\hline $\mathrm{EEA}_{\mathrm{it}}$ & $\begin{array}{l}-0.11 \\
(0.22)\end{array}$ & $\begin{array}{l}-0.038 \\
(0.23)\end{array}$ & $\begin{array}{l}-0.20 \\
(0.23)\end{array}$ & $\begin{array}{l}-0.53^{*} \\
(0.28)\end{array}$ & $\begin{array}{l}0.44^{*} \\
(0.25)\end{array}$ & $\begin{array}{l}1.03^{* * \star} \\
(0.30)\end{array}$ & $\begin{array}{c}0.73^{\star \star \star} \\
(0.28)\end{array}$ & $\begin{array}{l}0.72^{* *} \\
(0.30)\end{array}$ & $\begin{array}{l}0.99^{\star \star \star} \\
(0.30)\end{array}$ & $\begin{array}{l}1.02^{* \star \star} \\
(0.26)\end{array}$ \\
\hline $\mathrm{EEA}_{\mathrm{jt}}$ & $\begin{array}{l}0.73^{* *} \\
(0.37)\end{array}$ & $\begin{array}{l}0.65^{\star} \\
(0.37)\end{array}$ & $\begin{array}{l}1.15^{\star *} \\
(0.55)\end{array}$ & $\begin{array}{c}0.38 \\
(0.37)\end{array}$ & $\begin{array}{c}0.49 \\
(0.34)\end{array}$ & $\begin{array}{l}-0.015 \\
(0.37)\end{array}$ & $\begin{array}{c}-0.0041 \\
(0.36)\end{array}$ & $\begin{array}{c}0.13 \\
(0.36)\end{array}$ & $\begin{array}{l}-0.021 \\
(0.38)\end{array}$ & $\begin{array}{c}0.36 \\
(0.34)\end{array}$ \\
\hline $\mathrm{EEA}_{\mathrm{ijt}}$ & $\begin{array}{c}0.31 \\
(0.27)\end{array}$ & $\begin{array}{c}0.30 \\
(0.27)\end{array}$ & $\begin{array}{c}0.32 \\
(0.26)\end{array}$ & $\begin{array}{l}0.54^{\star *} \\
(0.26)\end{array}$ & $\begin{array}{c}0.16 \\
(0.22)\end{array}$ & $\begin{array}{c}0.11 \\
(0.20)\end{array}$ & $\begin{array}{l}0.099 \\
(0.20)\end{array}$ & $\begin{array}{c}0.10 \\
(0.20)\end{array}$ & $\begin{array}{c}0.10 \\
(0.21)\end{array}$ & $\begin{array}{l}0.098 \\
(0.19)\end{array}$ \\
\hline $\mathrm{EA}_{\mathrm{ijt}}$ & $\begin{array}{c}0.18 \\
(0.15)\end{array}$ & $\begin{array}{c}0.18 \\
(0.15)\end{array}$ & $\begin{array}{c}0.17 \\
(0.15)\end{array}$ & $\begin{array}{c}0.18 \\
(0.12)\end{array}$ & $\begin{array}{l}0.015 \\
(0.14)\end{array}$ & $\begin{array}{c}0.23 \\
(0.17)\end{array}$ & $\begin{array}{c}0.24 \\
(0.17)\end{array}$ & $\begin{array}{c}0.19 \\
(0.17)\end{array}$ & $\begin{array}{l}0.28^{\star} \\
(0.17)\end{array}$ & $\begin{array}{l}0.081 \\
(0.15)\end{array}$ \\
\hline $\mathrm{NAFTA}_{\mathrm{ij}}$ & $\begin{array}{c}-1.21^{* * *} \\
(0.29)\end{array}$ & $\begin{array}{c}-1.20^{* * *} \\
(0.29)\end{array}$ & $\begin{array}{c}-1.18^{* * *} \\
(0.29)\end{array}$ & $\begin{array}{c}-0.83^{* * *} \\
(0.27)\end{array}$ & $\begin{array}{c}-1.03^{\star * *} \\
(0.24)\end{array}$ & $\begin{array}{l}-1.10^{*} \\
(0.59)\end{array}$ & $\begin{array}{l}-1.11^{*} \\
(0.58)\end{array}$ & $\begin{array}{l}-1.14^{*} \\
(0.58)\end{array}$ & $\begin{array}{c}-1.36^{\star *} \\
(0.59)\end{array}$ & $\begin{array}{l}-0.97^{*} \\
(0.55)\end{array}$ \\
\hline$E P L_{\text {it }}$ & $\begin{array}{l}-0.19 \\
(0.18)\end{array}$ & $\begin{array}{l}-0.14 \\
(0.19)\end{array}$ & $\begin{array}{l}-0.28 \\
(0.20)\end{array}$ & $\begin{array}{l}-0.069 \\
(0.17)\end{array}$ & $\begin{array}{c}0.16 \\
(0.19)\end{array}$ & $\begin{array}{l}-0.11 \\
(0.19)\end{array}$ & $\begin{array}{l}-0.26 \\
(0.19)\end{array}$ & $\begin{array}{l}-0.34 \\
(0.21)\end{array}$ & $\begin{array}{l}-0.13 \\
(0.20)\end{array}$ & $\begin{array}{c}0.0040 \\
(0.18)\end{array}$ \\
\hline $\mathrm{EPL}_{\mathrm{jt}}$ & $\begin{array}{c}-0.60^{* *} \\
(0.29)\end{array}$ & $\begin{array}{l}-0.58^{\star *} \\
(0.28)\end{array}$ & $\begin{array}{l}-0.45 \\
(0.27)\end{array}$ & $\begin{array}{l}-0.53^{*} \\
(0.28)\end{array}$ & $\begin{array}{l}-0.48 \\
(0.36)\end{array}$ & $\begin{array}{l}-0.17 \\
(0.24)\end{array}$ & $\begin{array}{l}-0.12 \\
(0.24)\end{array}$ & $\begin{array}{l}0.045 \\
(0.25)\end{array}$ & $\begin{array}{l}-0.12 \\
(0.26)\end{array}$ & $\begin{array}{l}-0.31 \\
(0.24)\end{array}$ \\
\hline Tariff $_{i t}$ & $\begin{array}{l}-0.035 \\
(0.13)\end{array}$ & & & $\begin{array}{c}-0.0083 \\
(0.11)\end{array}$ & $\begin{array}{l}0.22^{*} \\
(0.12)\end{array}$ & $\begin{array}{c}0.48^{* * *} \\
(0.14)\end{array}$ & & & $\begin{array}{c}0.45^{\star * *} \\
(0.15)\end{array}$ & $\begin{array}{c}0.44^{* * *} \\
(0.13)\end{array}$ \\
\hline Tariff $_{j t}$ & $\begin{array}{c}0.14 \\
(0.12)\end{array}$ & & & $\begin{array}{l}0.19^{*} \\
(0.11)\end{array}$ & $\begin{array}{l}0.066 \\
(0.15)\end{array}$ & $\begin{array}{l}-0.13 \\
(0.13)\end{array}$ & & & $\begin{array}{l}-0.15 \\
(0.13)\end{array}$ & $\begin{array}{l}-0.23^{*} \\
(0.13)\end{array}$ \\
\hline FDI rest. index $i t$ & $\begin{array}{l}-0.25^{\star} \\
(0.14)\end{array}$ & & & $\begin{array}{c}-0.37^{\star *} \\
(0.19)\end{array}$ & $\begin{array}{l}0.039 \\
(0.13)\end{array}$ & $\begin{array}{l}-0.032 \\
(0.14) \\
\end{array}$ & & & $\begin{array}{l}-0.11 \\
(0.14)\end{array}$ & $\begin{array}{c}-0.0024 \\
(0.13)\end{array}$ \\
\hline$N$ & 2024 & 2024 & 2024 & 1958 & 2462 & 2024 & 2024 & 2024 & 1958 & 2462 \\
\hline Sample & $\begin{array}{c}0.894 \\
\text { OECD31 }\end{array}$ & $\begin{array}{c}0.891 \\
\text { OECD31 }\end{array}$ & $\begin{array}{c}0.891 \\
\text { OECD31 }\end{array}$ & $\begin{array}{c}0.914 \\
\text { OECD31 }\end{array}$ & $\begin{array}{c}0.837 \\
\text { OECD34 }\end{array}$ & $\begin{array}{c}0.816 \\
\text { OECD31 }\end{array}$ & $\begin{array}{c}0.816 \\
\text { OECD31 }\end{array}$ & $\begin{array}{c}0.817 \\
\text { OECD31 }\end{array}$ & $\begin{array}{c}0.818 \\
\text { OECD31 }\end{array}$ & $\begin{array}{c}0.820 \\
\text { OECD34 }\end{array}$ \\
\hline $\begin{array}{l}\text { Country and year } \\
\text { fixed effects }\end{array}$ & YES & YES & YES & YES & YES & YES & YES & YES & YES & YES \\
\hline $\begin{array}{l}\text { Controls for PMR levels } \\
\text { in seven sub-domains }\end{array}$ & NO & NO & YES & NO & NO & NO & NO & YES & NO & NO \\
\hline $\begin{array}{l}\text { Controls for size and } \\
\text { factor dissimilarities }\end{array}$ & NO & NO & NO & YES & NO & NO & NO & NO & YES & NO \\
\hline
\end{tabular}

Note: Asterisks $\left({ }^{*},{ }^{* *},{ }^{* * *}\right)$ indicate the significance level $(10 \%, 5 \%, 1 \%)$ of the coefficients. i denotes the inward country and $\mathrm{j}$ the outward country. Standard

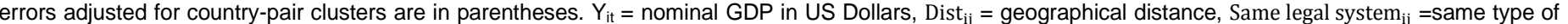

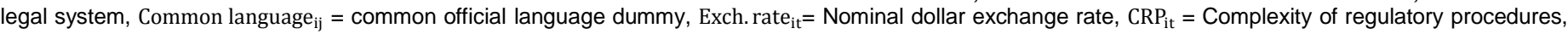
$\mathrm{EEA}_{\mathrm{it}}=$ European Economic Area dummy, $\mathrm{EA}_{\mathrm{ijt}}=$ euro area pair dummy, NAFTA $\mathrm{ij}_{\mathrm{ij}}=$ North American Free Trade Agreement pair dummy, PMR $_{\mathrm{it}}=\mathrm{Strictness}_{\mathrm{i}}$

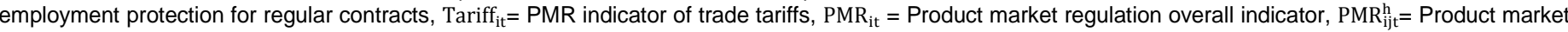
regulation indicator heterogeneity. Poisson = Poisson pseudo-maximum likelihood. OECD31 refers to all OECD countries except Hungary, Luxembourg and the Netherlands that have the highest share of special purpose entities, OEC34 refers to all OECD countries. 
Table 2. FDI determinants: Robustness check with time-varying fixed effects

\begin{tabular}{|c|c|c|c|c|c|c|c|c|}
\hline Estimation method & $\begin{array}{c}(1) \\
\text { Poisson }\end{array}$ & $\begin{array}{c}(2) \\
\text { Poisson }\end{array}$ & $\begin{array}{c}\text { (3) } \\
\text { Poisson }\end{array}$ & $\begin{array}{c}\text { (4) } \\
\text { Poisson }\end{array}$ & $\begin{array}{c}(5) \\
\text { Linear }\end{array}$ & $\begin{array}{c}(6) \\
\text { Linear }\end{array}$ & $\begin{array}{c}(7) \\
\text { Linear }\end{array}$ & $\begin{array}{c}(8) \\
\text { Linear }\end{array}$ \\
\hline $\ln \left(\right.$ dist $\left._{i j}\right)$ & $\begin{array}{c}-0.65^{\star * *} \\
(0.099)\end{array}$ & $\begin{array}{c}-0.59^{* * *} \\
(0.091)\end{array}$ & $\begin{array}{c}-0.57^{* \star *} \\
(0.086)\end{array}$ & $\begin{array}{c}-0.56^{\star * *} \\
(0.11)\end{array}$ & $\begin{array}{c}-1.17^{* * *} \\
(0.11)\end{array}$ & $\begin{array}{c}-1.20^{\star \star \star} \\
(0.11)\end{array}$ & $\begin{array}{c}-1.04^{* * *} \\
(0.10)\end{array}$ & $\begin{array}{c}-1.14^{* * *} \\
(0.11)\end{array}$ \\
\hline Contiguity $_{\mathrm{ij}}$ & $\begin{array}{c}0.15 \\
(0.15)\end{array}$ & $\begin{array}{c}0.19 \\
(0.13)\end{array}$ & $\begin{array}{l}0.21^{*} \\
(0.13)\end{array}$ & $\begin{array}{c}0.17 \\
(0.16)\end{array}$ & $\begin{array}{l}-0.19 \\
(0.23)\end{array}$ & $\begin{array}{l}-0.21 \\
(0.24)\end{array}$ & $\begin{array}{c}-0.0096 \\
(0.21)\end{array}$ & $\begin{array}{l}-0.19 \\
(0.24)\end{array}$ \\
\hline Same legal system $_{\mathrm{ij}}$ & $\begin{array}{l}0.45^{\star \star *} \\
(0.095)\end{array}$ & $\begin{array}{l}0.43^{* * *} \\
(0.085)\end{array}$ & $\begin{array}{l}0.30^{* * *} \\
(0.084)\end{array}$ & $\begin{array}{l}0.48^{* * *} \\
(0.098)\end{array}$ & $\begin{array}{c}0.64^{* * *} \\
(0.12)\end{array}$ & $\begin{array}{c}0.64^{* * *} \\
(0.12)\end{array}$ & $\begin{array}{l}0.68^{* * *} \\
(0.10)\end{array}$ & $\begin{array}{c}0.69^{* * *} \\
(0.12)\end{array}$ \\
\hline Common language $_{i j}$ & $\begin{array}{c}0.14 \\
(0.14)\end{array}$ & $\begin{array}{c}0.19 \\
(0.13)\end{array}$ & $\begin{array}{l}0.073 \\
(0.11)\end{array}$ & $\begin{array}{l}0.015 \\
(0.15)\end{array}$ & $\begin{array}{c}0.64^{* \star *} \\
(0.22)\end{array}$ & $\begin{array}{c}0.75^{\star * *} \\
(0.22)\end{array}$ & $\begin{array}{l}0.48^{\star *} \\
(0.20)\end{array}$ & $\begin{array}{l}0.54^{* *} \\
(0.21)\end{array}$ \\
\hline $\mathrm{PMR}_{i j t}^{h}$ & $\begin{array}{c}-1.64^{\star *} \\
(0.77)\end{array}$ & $\begin{array}{l}-0.67 \\
(0.68)\end{array}$ & $\begin{array}{c}-3.04^{* \star *} \\
(0.71)\end{array}$ & $\begin{array}{c}-2.29^{\star * *} \\
(0.76)\end{array}$ & $\begin{array}{c}-2.31^{* * *} \\
(0.81)\end{array}$ & $\begin{array}{c}-1.84^{\star *} \\
(0.83)\end{array}$ & $\begin{array}{c}-2.24^{* * *} \\
(0.74)\end{array}$ & $\begin{array}{c}-2.08^{\star *} \\
(0.81)\end{array}$ \\
\hline $\mathrm{EEA}_{\mathrm{ijt}}$ & $\begin{array}{c}0.35 \\
(0.25)\end{array}$ & $\begin{array}{l}0.56^{* *} \\
(0.25)\end{array}$ & $\begin{array}{c}0.23 \\
(0.22)\end{array}$ & $\begin{array}{c}0.41 \\
(0.26)\end{array}$ & $\begin{array}{c}-0.0055 \\
(0.21)\end{array}$ & $\begin{array}{c}0.0037 \\
(0.21)\end{array}$ & $\begin{array}{l}-0.015 \\
(0.19)\end{array}$ & $\begin{array}{l}-0.086 \\
(0.20)\end{array}$ \\
\hline $\mathrm{EA}_{\mathrm{ijt}}$ & $\begin{array}{c}0.17 \\
(0.16)\end{array}$ & $\begin{array}{c}0.19 \\
(0.13)\end{array}$ & $\begin{array}{l}-0.050 \\
(0.15)\end{array}$ & $\begin{array}{c}0.13 \\
(0.17)\end{array}$ & $\begin{array}{c}0.26 \\
(0.20)\end{array}$ & $\begin{array}{c}0.31 \\
(0.20)\end{array}$ & $\begin{array}{l}0.080 \\
(0.18)\end{array}$ & $\begin{array}{c}0.28 \\
(0.21)\end{array}$ \\
\hline NAFTA $_{i j}$ & $\begin{array}{c}-1.40^{* \star *} \\
(0.30)\end{array}$ & $\begin{array}{c}-0.96^{\star \star \star} \\
(0.28)\end{array}$ & $\begin{array}{c}-1.21^{* \star *} \\
(0.25)\end{array}$ & $\begin{array}{c}-1.19^{* \star *} \\
(0.32)\end{array}$ & $\begin{array}{l}-1.00^{*} \\
(0.60)\end{array}$ & $\begin{array}{c}-1.26^{\star *} \\
(0.61)\end{array}$ & $\begin{array}{l}-0.91 \\
(0.57)\end{array}$ & $\begin{array}{l}-0.67 \\
(0.67)\end{array}$ \\
\hline $\mathrm{HCD}_{\mathrm{ijt}}$ & & $\begin{array}{c}0.50 \\
(0.40)\end{array}$ & & & & $\begin{array}{c}0.28 \\
(0.50)\end{array}$ & & \\
\hline $\mathrm{FD}_{\mathrm{ijt}}$ & & $\begin{array}{c}-0.87^{* * *} \\
(0.15)\end{array}$ & & & & $\begin{array}{c}0.16 \\
(0.11)\end{array}$ & & \\
\hline $\mathrm{SS}_{\mathrm{ijt}}$ & & $\begin{array}{l}0.42^{* * *} \\
(0.055)\end{array}$ & & & & $\begin{array}{l}0.35^{\star * *} \\
(0.068)\end{array}$ & & \\
\hline$N$ & 2209 & 2143 & 2705 & 1746 & 2209 & 2143 & 2705 & 1746 \\
\hline$R^{2}$ & 0.913 & 0.932 & 0.917 & 0.925 & 0.834 & 0.837 & 0.840 & 0.842 \\
\hline Sample & OECD31 & OECD31 & OECD34 & OECD31 & OECD31 & OECD31 & OECD34 & OECD31 \\
\hline $\begin{array}{l}\text { Country and year } \\
\text { time-varying fixed } \\
\text { effects }\end{array}$ & YES & YES & YES & YES & YES & YES & YES & YES \\
\hline End of sample & 2013 & 2013 & 2013 & 2008 & 2013 & 2013 & 2013 & 2008 \\
\hline
\end{tabular}

Note: Asterisks $\left({ }^{*},{ }^{* \star},{ }^{* \star *}\right)$ indicate the significance level $(10 \%, 5 \%, 1 \%)$ of the coefficients. i denotes the inward country and $j$ the outward country.

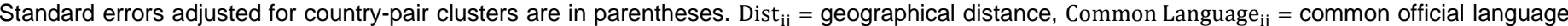

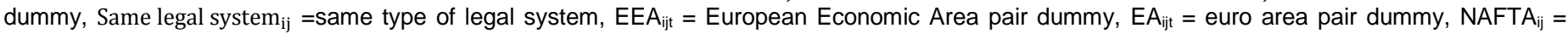
North American Free Trade Agreement pair dummy, $\mathrm{PMR}_{\mathrm{it}}=$ Strictness of employment protection for regular contracts, Tariff ${ }_{\mathrm{it}}=\mathrm{PMR}_{\mathrm{indicator}}$ of trade tariffs, $\mathrm{PMR}_{\mathrm{it}}=$ Product market regulation overall indicator, $\mathrm{PMR}_{\mathrm{ijt}}^{\mathrm{h}}=$ Product market regulation indicator heterogeneity, $\mathrm{HCD}_{\mathrm{ij}}=\mathrm{human}_{\mathrm{C}} \mathrm{capital}$ dissimilarity (absolute difference in the average number of years of schooling), $\mathrm{FD}_{\mathrm{ij}}=$ factor dissimilarity (difference in GDP per capita used as a proxy for the difference in the stock of capital), $\mathrm{SS}_{\mathrm{ijt}}=$ size similarity. Poisson $=$ Poisson pseudo-maximum likelihood. OECD31 refers to all OECD countries except Hungary, Luxembourg and the Netherlands that have the highest share of special purpose entities, OEC34 refers to all OECD countries.

\section{Heterogeneity effect by regulatory domain}

42. Sub-level measures of the heterogeneity of product market regulations are used to better understand which aspects of heterogeneity matter most for FDI decisions. Among the three main domains covered by the PMR indicator (Figure 1), the analysis focuses on the heterogeneity of state control and on the heterogeneity of barriers to entrepreneurship. There is no evidence of any role of divergence in barriers to trade and investment, an aspect for which it is rather the level of stringency that matters. ${ }^{8}$ In a first step, five sub-domains of the heterogeneity indicator ("Public Ownership", "Involvement in business operations", "Complexity of regulatory procedures", "Administrative burdens on start-ups" and "Regulatory protection of incumbents") are included, revealing that two aspects have a negative effect in each specification: the involvement in business operations and the regulatory protection of incumbents (Table A1.5). In a second step, these two sub-domains are decomposed at a lower level, and the three others are dropped for the sake of parsimony (Table 3). This last step shows that the heterogeneity of barriers in services and the heterogeneity of antitrust exemptions have a particularly robust adverse effect on FDI. The heterogeneity of barriers in network sectors also has a negative effect that is significant in all

8. Regressions (not reported here) with these three main domains show no effect of the heterogeneity of barriers to trade and investment. 


\section{$\mathrm{ECO} / \mathrm{WKP}(2015) 86$}

linear regressions. Last, there is also a negative effect of the heterogeneity of command and control regulation, and the effect is significant in specifications with the Poisson estimator.

Table 3. The role of heterogeneity by selected low-level regulation domain

\begin{tabular}{|c|c|c|c|c|c|c|c|c|c|c|}
\hline Estimation method & $\begin{array}{c}(1) \\
\text { Poisson }\end{array}$ & $\begin{array}{c}(2) \\
\text { Poisson }\end{array}$ & $\begin{array}{c}(3) \\
\text { Poisson }\end{array}$ & $\begin{array}{c}(4) \\
\text { Poisson }\end{array}$ & $\begin{array}{c}(5) \\
\text { Poisson } \\
\end{array}$ & $\begin{array}{c}(6) \\
\text { Linear }\end{array}$ & $\begin{array}{c}(7) \\
\text { Linear }\end{array}$ & $\begin{array}{c}(8) \\
\text { Linear }\end{array}$ & $\begin{array}{c}(9) \\
\text { Linear }\end{array}$ & $\begin{array}{c}(10) \\
\text { Linear }\end{array}$ \\
\hline Price controls $\mathrm{s}_{\mathrm{ijt}}^{\mathrm{h}}$ & $\begin{array}{l}0.37^{*} \\
(0.21)\end{array}$ & $\begin{array}{l}0.36^{*} \\
(0.20)\end{array}$ & $\begin{array}{l}0.53^{* *} \\
(0.21)\end{array}$ & $\begin{array}{l}0.096 \\
(0.20)\end{array}$ & $\begin{array}{c}0.34 \\
(0.24)\end{array}$ & $\begin{array}{c}0.28 \\
(0.33)\end{array}$ & $\begin{array}{c}0.26 \\
(0.32)\end{array}$ & $\begin{array}{c}0.31 \\
(0.33)\end{array}$ & $\begin{array}{c}0.24 \\
(0.33)\end{array}$ & $\begin{array}{l}0.45 \\
(0.30)\end{array}$ \\
\hline $\begin{array}{c}\text { Command and } \\
\text { control regulation }_{\mathrm{ijt}}^{\mathrm{h}}\end{array}$ & $\begin{array}{l}-0.50^{*} \\
(0.27)\end{array}$ & $\begin{array}{l}-0.51^{*} \\
(0.27)\end{array}$ & $\begin{array}{l}-0.45^{\star} \\
(0.27)\end{array}$ & $\begin{array}{l}-0.57^{\star *} \\
(0.24)\end{array}$ & $\begin{array}{l}-0.53^{* *} \\
(0.24)\end{array}$ & $\begin{array}{l}-0.19 \\
(0.33)\end{array}$ & $\begin{array}{l}-0.15 \\
(0.33)\end{array}$ & $\begin{array}{l}-0.18 \\
(0.34)\end{array}$ & $\begin{array}{r}-0.075 \\
(0.34)\end{array}$ & $\begin{array}{l}-0.33 \\
(0.29)\end{array}$ \\
\hline Legal barriers $_{\mathrm{ijt}}^{\mathrm{h}}$ & $\begin{array}{l}0.022 \\
(0.52)\end{array}$ & $\begin{array}{l}0.15 \\
(0.53)\end{array}$ & $\begin{array}{c}-0.0025 \\
(0.57)\end{array}$ & $\begin{array}{c}0.40 \\
(0.42)\end{array}$ & $\begin{array}{c}0.39 \\
(0.50)\end{array}$ & $\begin{array}{l}0.80 \\
(0.56)\end{array}$ & $\begin{array}{c}0.74 \\
(0.55)\end{array}$ & $\begin{array}{c}0.91 \\
(0.60)\end{array}$ & $\begin{array}{l}0.65 \\
(0.56)\end{array}$ & $\begin{array}{l}0.83 \\
(0.52)\end{array}$ \\
\hline Antitrust exempt ${ }_{\mathrm{ijt}}^{\mathrm{h}}$ & $\begin{array}{l}-0.25^{\star * *} \\
(0.064)\end{array}$ & $\begin{array}{l}-0.25^{\star * *} \\
(0.065)\end{array}$ & $\begin{array}{l}-0.26^{\star * *} \\
(0.064)\end{array}$ & $\begin{array}{l}-0.13^{\star *} \\
(0.060)\end{array}$ & $\begin{array}{l}-0.32^{\star * *} \\
(0.064)\end{array}$ & $\begin{array}{l}-0.27^{* * *} \\
(0.085)\end{array}$ & $\begin{array}{c}-0.26^{\star * *} \\
(0.086)\end{array}$ & $\begin{array}{l}-0.25^{\star * *} \\
(0.085)\end{array}$ & $\begin{array}{l}-0.19^{\star *} \\
(0.084)\end{array}$ & $\begin{array}{c}-0.30^{* * *} \\
(0.083)\end{array}$ \\
\hline Network sectors ${ }_{i j t}^{h}$ & $\begin{array}{l}-0.47 \\
(0.33)\end{array}$ & $\begin{array}{l}-0.47 \\
(0.32)\end{array}$ & $\begin{array}{l}-0.37 \\
(0.31)\end{array}$ & $\begin{array}{l}-0.24 \\
(0.28)\end{array}$ & $\begin{array}{l}0.032 \\
(0.30)\end{array}$ & $\begin{array}{l}-0.80^{\star *} \\
(0.36)\end{array}$ & $\begin{array}{l}-0.77^{\star *} \\
(0.35)\end{array}$ & $\begin{array}{l}-0.78^{\star *} \\
(0.36)\end{array}$ & $\begin{array}{l}-0.76^{\star *} \\
(0.36)\end{array}$ & $\begin{array}{l}-0.56^{*} \\
(0.31)\end{array}$ \\
\hline Services $_{\mathrm{ijt}}^{\mathrm{h}}$ & $\begin{array}{c}-1.17^{* * *} \\
(0.37)\end{array}$ & $\begin{array}{c}-1.19^{* * *} \\
(0.37)\end{array}$ & $\begin{array}{c}-1.25^{\star * *} \\
(0.37)\end{array}$ & $\begin{array}{c}-1.05^{\star \star \star} \\
(0.29)\end{array}$ & $\begin{array}{c}-1.55^{\star * \star} \\
(0.31)\end{array}$ & $\begin{array}{c}-1.42^{\star * \star} \\
(0.37)\end{array}$ & $\begin{array}{c}-1.43^{\star * *} \\
(0.38)\end{array}$ & $\begin{array}{c}-1.46^{\star * *} \\
(0.38)\end{array}$ & $\begin{array}{c}-1.36^{\star * \star} \\
(0.39)\end{array}$ & $\begin{array}{c}-1.21^{* * *} \\
(0.33)\end{array}$ \\
\hline $\mathrm{PMR}_{\text {it }}$ & & $\begin{array}{l}-0.26 \\
(0.18)\end{array}$ & & & & & $\begin{array}{c}0.28 \\
(0.26)\end{array}$ & & & \\
\hline $\mathrm{PMR}_{\mathrm{jt}}$ & & $\begin{array}{c}0.13 \\
(0.17)\end{array}$ & & & & & $\begin{array}{l}-0.40 \\
(0.25)\end{array}$ & & & \\
\hline $\mathrm{CRP}_{\text {it }}$ & $\begin{array}{l}-0.15^{\star \star *} \\
(0.052)\end{array}$ & & $\begin{array}{l}-0.17^{* * *} \\
(0.050)\end{array}$ & $\begin{array}{l}-0.16^{\star * *} \\
(0.045)\end{array}$ & $\begin{array}{l}-0.15^{\star * *} \\
(0.053)\end{array}$ & $\begin{array}{c}0.030 \\
(0.074) \\
\end{array}$ & & $\begin{array}{c}0.014 \\
(0.072)\end{array}$ & $\begin{array}{l}0.0026 \\
(0.072)\end{array}$ & $\begin{array}{c}-0.0046 \\
(0.064)\end{array}$ \\
\hline $\mathrm{N}$ & 2024 & 2024 & 2024 & 1958 & 2462 & 2024 & 2024 & 2024 & 1958 & 2462 \\
\hline Sample $\quad \mathrm{R}^{2}$ & $\begin{array}{c}0.900 \\
\text { OECD31 }\end{array}$ & $\begin{array}{c}0.899 \\
\text { OECD31 }\end{array}$ & $\begin{array}{c}0.898 \\
\text { OECD31 }\end{array}$ & $\begin{array}{c}0.920 \\
\text { OECD31 }\end{array}$ & $\begin{array}{c}0.849 \\
\text { OECD34 }\end{array}$ & $\begin{array}{c}0.819 \\
\text { OECD31 }\end{array}$ & $\begin{array}{c}0.818 \\
\text { OECD31 }\end{array}$ & $\begin{array}{c}0.819 \\
\text { OECD31 }\end{array}$ & $\begin{array}{c}0.820 \\
\text { OECD31 }\end{array}$ & $\begin{array}{c}0.823 \\
\text { OECD34 }\end{array}$ \\
\hline $\begin{array}{l}\text { Country and year } \\
\text { fixed effects }\end{array}$ & YES & YES & YES & YES & YES & YES & YES & YES & YES & YES \\
\hline $\begin{array}{l}\text { Controls for PMR } \\
\text { levels in } 7 \text { sub- } \\
\text { domains }\end{array}$ & NO & $\mathrm{NO}$ & YES & $\mathrm{NO}$ & NO & NO & NO & YES & NO & $\mathrm{NO}$ \\
\hline $\begin{array}{l}\text { Controls for size and } \\
\text { factor dissimilarities }\end{array}$ & NO & NO & $\mathrm{NO}$ & YES & NO & NO & NO & NO & YES & NO \\
\hline
\end{tabular}

Note: Asterisks $\left({ }^{*},{ }^{* \star},{ }^{* *}\right)$ indicate the significance level $(10 \%, 5 \%, 1 \%)$ of the coefficients. i denotes the inward country and $j$ the outward country. Standard errors adjusted for country-pair clusters are in parentheses. The superscript "h" stands for "heterogeneity". $\mathrm{CRP}_{\text {it }}=$ Complexity of regulatory procedures. Sector-specific heterogeneity measures are added on top of the baseline regressions shown in Table 1. Poisson = Poisson pseudo-maximum likelihood. OECD31 refers to all OECD countries except Hungary, Luxembourg and the Netherlands that have the highest share of special purpose entities, OEC34 refers to all OECD countries.

\section{Employment protection legislation}

43. The strictness of employment protection for regular contracts has a large negative effect on both outward and inward FDI. Stringent employment protection legislation slows down the reallocation process (Haltiwanger et al., 2006; Andrews and Criscuolo, 2013): it can decrease both outward and inward FDI by impeding the restructuring associated with mergers and acquisitions. It can also decrease outward FDI by making firms less profitable and dynamic and hence less likely to expand abroad. Levine et al. (2015) find that acquiring firms enjoy smaller stock returns and profits when targets are in countries with stronger labour protection regulations, especially when the target is in a labour-intensive or high labour-volatility industry. In addition, higher employment protection increases the risks beard by the foreign investor, which can deter inward FDI. Finally, it can reduce productivity growth (Autor et al., 2007; Bassanini et al., 2009) and hence can reduce FDI through a decrease of the size of the economy.

44. A large effect is identified with regressions over a longer time span (Table 4). As the index of employment protection legislation is not changing much, the baseline regression over 1998 to 2013 can only provide tentative evidence of this EPL stringency effect. In countries that are far away from best practice such as Chile, the Netherlands, Slovenia or Sweden, an ambitious reform package aiming at aligning the employment protection legislation to the average stance of the top half of best performers could increase FDI by about $40 \%$ (Figure 4). An alternative angle to assess the effect of employment protection legislation on FDI is to consider a reform in all OECD countries but of a smaller size. This 
alternative scenario takes into account both of the effect on the host and on the investing countries: each country benefits from reforms done elsewhere. For instance, if all countries ease employment protection as it was done between 2003 and 2013 in those 15 OECD countries where the EPL index has decreased, FDI could increase by about $25 \%$.

Table 4. FDI determinants: Results with a 1990-2013 sample

\begin{tabular}{|c|c|c|c|c|c|c|c|c|c|c|}
\hline Estimation method & $\begin{array}{c}\text { (1) } \\
\text { Posen }\end{array}$ & $\begin{array}{c}(2) \\
\end{array}$ & $\begin{array}{c}\text { (3) } \\
\text { (3) }\end{array}$ & $\begin{array}{c}(4) \\
\end{array}$ & $\begin{array}{c}(5) \\
(5)\end{array}$ & (6) & $(7)$ & $(8)$ & (9) & $(10)$ \\
\hline$\frac{\text { Estimation method }}{\mathrm{EPL}_{\mathrm{it}}}$ & $\begin{array}{c}\text { Poisson } \\
-0.26^{* *} \\
(0.11)\end{array}$ & $\begin{array}{c}\text { Poisson } \\
-0.54^{\star \star *} \\
(0.20)\end{array}$ & $\begin{array}{c}\text { Poisson } \\
-0.15 \\
(0.12)\end{array}$ & $\begin{array}{c}\text { Poisson } \\
-0.12 \\
(0.11)\end{array}$ & $\begin{array}{c}\text { Poisson } \\
-0.20^{*} \\
(0.11)\end{array}$ & $\begin{array}{c}\text { Linear } \\
-0.45^{\star \star \star} \\
(0.14)\end{array}$ & $\begin{array}{c}\text { Linear } \\
-0.63^{* * *} \\
(0.21)\end{array}$ & $\begin{array}{l}\text { Linear } \\
-0.27^{* *} \\
(0.13)\end{array}$ & $\begin{array}{l}\text { Linear } \\
-0.27^{\star *} \\
(0.13)\end{array}$ & $\begin{array}{c}\text { Linear } \\
-0.45^{\star * *} \\
(0.13)\end{array}$ \\
\hline $\mathrm{EPL}_{\mathrm{jt}}$ & $\begin{array}{c}-0.58^{\star * *} \\
(0.16)\end{array}$ & $\begin{array}{c}-0.59^{* * *} \\
(0.21)\end{array}$ & $\begin{array}{c}-0.67^{\star \star \star *} \\
(0.18)\end{array}$ & $\begin{array}{c}-0.62^{* * *} \\
(0.17)\end{array}$ & $\begin{array}{c}-0.51^{* * *} \\
(0.19)\end{array}$ & $\begin{array}{l}-0.11 \\
(0.17)\end{array}$ & $\begin{array}{l}-0.51^{*} \\
(0.30)\end{array}$ & $\begin{array}{l}-0.011 \\
(0.17)\end{array}$ & $\begin{array}{l}0.058 \\
(0.17)\end{array}$ & $\begin{array}{l}-0.16 \\
(0.16)\end{array}$ \\
\hline $\mathrm{EEA}_{\mathrm{it}}$ & $\begin{array}{c}0.23 \\
(0.17)\end{array}$ & $\begin{array}{c}0.21 \\
(0.17)\end{array}$ & $\begin{array}{c}0.24 \\
(0.21)\end{array}$ & $\begin{array}{c}-0.18 \\
(0.22)\end{array}$ & $\begin{array}{c}0.25 \\
(0.15)\end{array}$ & $\begin{array}{c}0.51^{* \star *} \\
(0.18)\end{array}$ & $\begin{array}{c}0.50^{* \star *} \\
(0.16)\end{array}$ & $\begin{array}{l}0.36^{*} \\
(0.19)\end{array}$ & $\begin{array}{l}0.31^{*} \\
(0.19)\end{array}$ & $\begin{array}{l}0.38^{* *} \\
(0.15)\end{array}$ \\
\hline $\mathrm{EEA}_{\mathrm{jt}}$ & $\begin{array}{c}0.81^{* * *} \\
(0.27)\end{array}$ & $\begin{array}{l}0.44^{*} \\
(0.26)\end{array}$ & $\begin{array}{c}0.86^{* * *} \\
(0.30)\end{array}$ & $\begin{array}{c}0.37 \\
(0.28)\end{array}$ & $\begin{array}{l}0.67^{\star *} \\
(0.28)\end{array}$ & $\begin{array}{l}0.031 \\
(0.25)\end{array}$ & $\begin{array}{l}-0.11 \\
(0.23)\end{array}$ & $\begin{array}{l}-0.087 \\
(0.26)\end{array}$ & $\begin{array}{l}-0.17 \\
(0.26)\end{array}$ & $\begin{array}{l}0.43^{*} \\
(0.22)\end{array}$ \\
\hline $\mathrm{EEA}_{\mathrm{ijt}}$ & $\begin{array}{c}0.35 \\
(0.25)\end{array}$ & $\begin{array}{c}0.37 \\
(0.26)\end{array}$ & $\begin{array}{l}0.047 \\
(0.26)\end{array}$ & $\begin{array}{c}0.23 \\
(0.25)\end{array}$ & $\begin{array}{c}0.23 \\
(0.21)\end{array}$ & $\begin{array}{c}0.22 \\
(0.18)\end{array}$ & $\begin{array}{c}0.14 \\
(0.18)\end{array}$ & $\begin{array}{c}0.13 \\
(0.19)\end{array}$ & $\begin{array}{c}0.14 \\
(0.19)\end{array}$ & $\begin{array}{c}0.19 \\
(0.17)\end{array}$ \\
\hline$E A_{i t}$ & $\begin{array}{l}-0.038 \\
(0.099)\end{array}$ & $\begin{array}{l}-0.012 \\
(0.097)\end{array}$ & $\begin{array}{l}-0.23^{*} \\
(0.12)\end{array}$ & $\begin{array}{l}-0.22^{*} \\
(0.11)\end{array}$ & $\begin{array}{c}-0.0087 \\
(0.11)\end{array}$ & $\begin{array}{l}-0.058 \\
(0.11)\end{array}$ & $\begin{array}{l}-0.17 \\
(0.13)\end{array}$ & $\begin{array}{l}-0.22^{*} \\
(0.13)\end{array}$ & $\begin{array}{c}-0.28^{\star *} \\
(0.13)\end{array}$ & $\begin{array}{l}0.033 \\
(0.11)\end{array}$ \\
\hline$E A_{j t}$ & $\begin{array}{l}0.39^{* \star *} \\
(0.097)\end{array}$ & $\begin{array}{l}0.37^{\star \star \star} \\
(0.096)\end{array}$ & $\begin{array}{c}0.17 \\
(0.11)\end{array}$ & $\begin{array}{l}0.21^{\star *} \\
(0.10)\end{array}$ & $\begin{array}{l}0.40^{\star * *} \\
(0.10)\end{array}$ & $\begin{array}{c}0.48^{* * *} \\
(0.13)\end{array}$ & $\begin{array}{c}0.53^{* \star *} \\
(0.13)\end{array}$ & $\begin{array}{l}0.34^{* *} \\
(0.14)\end{array}$ & $\begin{array}{l}0.29^{* *} \\
(0.14)\end{array}$ & $\begin{array}{c}0.49^{* \star *} \\
(0.12)\end{array}$ \\
\hline$E A_{i j t}$ & $\begin{array}{l}0.28^{*} \\
(0.16)\end{array}$ & $\begin{array}{c}0.22 \\
(0.16)\end{array}$ & $\begin{array}{l}0.28^{*} \\
(0.15)\end{array}$ & $\begin{array}{c}0.22 \\
(0.14)\end{array}$ & $\begin{array}{c}0.13 \\
(0.15)\end{array}$ & $\begin{array}{c}0.23 \\
(0.16)\end{array}$ & $\begin{array}{c}0.21 \\
(0.17)\end{array}$ & $\begin{array}{c}0.23 \\
(0.17)\end{array}$ & $\begin{array}{c}0.27 \\
(0.17)\end{array}$ & $\begin{array}{l}0.022 \\
(0.15)\end{array}$ \\
\hline NAFTA $_{i j t}$ & $\begin{array}{c}-0.77^{\star *} \\
(0.30)\end{array}$ & $\begin{array}{c}-0.87^{* * *} \\
(0.33)\end{array}$ & $\begin{array}{c}-1.02^{* \star *} \\
(0.29)\end{array}$ & $\begin{array}{c}-0.73^{\star * \star} \\
(0.25)\end{array}$ & $\begin{array}{l}-0.50^{* *} \\
(0.25)\end{array}$ & $\begin{array}{l}-0.56 \\
(0.54)\end{array}$ & $\begin{array}{l}-0.28 \\
(0.63)\end{array}$ & $\begin{array}{l}-0.044 \\
(0.47)\end{array}$ & $\begin{array}{l}-0.25 \\
(0.49)\end{array}$ & $\begin{array}{l}-0.48 \\
(0.53)\end{array}$ \\
\hline $\mathrm{ETCR}_{\text {it }}$ & & & $\begin{array}{l}-0.15^{\star} \\
(0.078)\end{array}$ & $\begin{array}{c}-0.12 \\
(0.074)\end{array}$ & & & & $\begin{array}{c}-0.30^{* * *} \\
(0.085)\end{array}$ & $\begin{array}{c}-0.36^{* \star *} \\
(0.085)\end{array}$ & \\
\hline $\mathrm{ETCR}_{\mathrm{jt}}$ & & & $\begin{array}{l}-0.18^{\star *} \\
(0.080)\end{array}$ & $\begin{array}{l}-0.13^{*} \\
(0.074)\end{array}$ & & & & $\begin{array}{c}-0.25^{\star *} \\
(0.10)\end{array}$ & $\begin{array}{c}-0.25^{\star *} \\
(0.10)\end{array}$ & \\
\hline $\mathrm{N}$ & 9634 & 6036 & 8917 & 8761 & 11584 & 9634 & 6036 & 8917 & 8761 & 11584 \\
\hline $\mathrm{R}^{2}$ & 0.917 & 0.920 & 0.831 & 0.854 & 0.806 & 0.821 & 0.835 & 0.807 & 0.810 & 0.826 \\
\hline $\begin{array}{l}\text { End of sample } \\
\text { Sample }\end{array}$ & $\begin{array}{c}2013 \\
\text { OECD31 }\end{array}$ & $\begin{array}{l}2008 \\
\text { OECD31 }\end{array}$ & $\begin{array}{l}2013 \\
\text { OECD31 }\end{array}$ & $\begin{array}{c}2013 \\
\text { OECD31 }\end{array}$ & $\begin{array}{l}2013 \\
\text { OECD34 }\end{array}$ & $\begin{array}{c}2013 \\
\text { OECD31 }\end{array}$ & $\begin{array}{l}2008 \\
\text { OECD31 }\end{array}$ & $\begin{array}{l}2013 \\
\text { OECD31 }\end{array}$ & $\begin{array}{l}2013 \\
\text { OECD31 }\end{array}$ & $\begin{array}{c}2013 \\
\text { OECD34 }\end{array}$ \\
\hline $\begin{array}{l}\text { Country and year } \\
\text { fixed effects }\end{array}$ & YES & YES & YES & YES & YES & YES & YES & YES & YES & YES \\
\hline $\begin{array}{l}\text { Controls for size and } \\
\text { factor dissimilarities }\end{array}$ & NO & NO & NO & YES & NO & NO & NO & NO & YES & NO \\
\hline
\end{tabular}

Note: Asterisks $\left({ }^{*},{ }^{* *},{ }^{* *}\right)$ indicate the significance level $(10 \%, 5 \%, 1 \%)$ of the coefficients. i denotes the inward country and $j$ the outward country. Standard errors adjusted for country-pair clusters are in parentheses. Regulatory variables are added on top of macroeconomic and geographical variables, see table A1.7 for detailed regression results. $E E A_{i t}=$ European Economic Area dummy, $\mathrm{EA}_{\mathrm{ijt}}=$ euro area pair dummy, $\mathrm{NAFTA}_{\mathrm{ijt}}=$ North American Free Trade Agreement pair dummy, $\mathrm{EPL}_{\mathrm{it}}=\mathrm{Strictness}_{\mathrm{i}}$ of employment protection for regular contracts, $E T C R_{\mathrm{it}}=$ Electricity, Transport and Communication Regulation indicator. Poisson = Poisson pseudomaximum likelihood. 
Figure 4. Reforms of employment protection legislation can boost FDI

FDI effect (in per cent) of easing employment protection towards the average of the top half of best performers

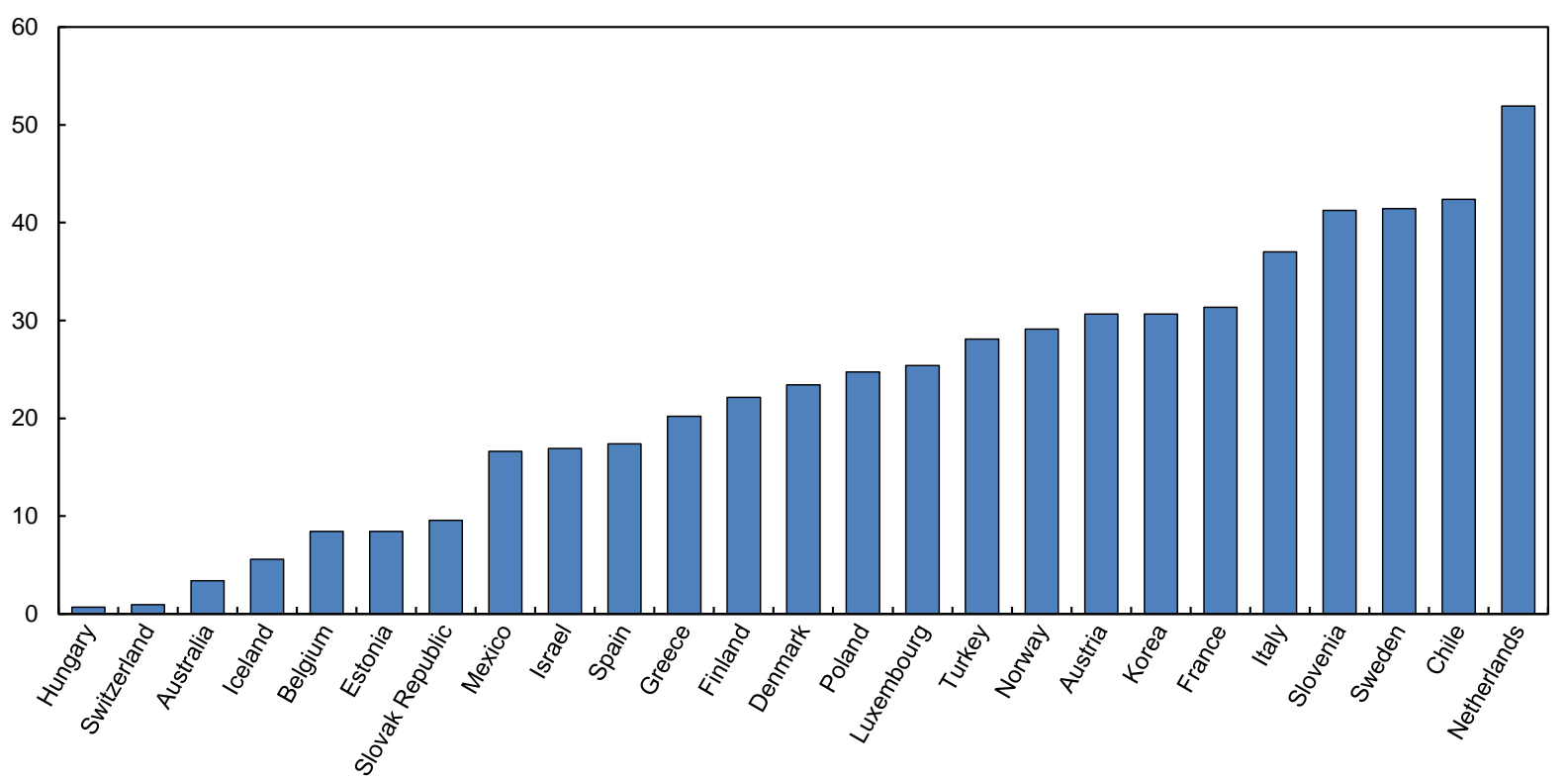

Note: The differences across countries reflect the difference in employment protection legislation observed in 2013. The countries where the employment protection legislation is less stringent than the average of the top half are not shown.

Source: OECD calculation based on the average effect of the employment protection in the host country on FDI stocks as measured in the Poisson models shown in Table 4.

\section{Complexity of regulatory procedures and FDI restrictiveness impede inward FDI}

45. There are two specific regulatory domains for which the regulatory stringency itself impedes inward FDI: the complexity of regulatory procedures and explicit barriers to FDI as one would expect (Table 1). As regards the complexity of regulatory procedures, the significant negative result suggests that the complexity of regulation implies a higher cost for foreign firms that are less familiar with the regulatory framework then the local firms. If countries that have complex regulatory procedures move towards the average of the top half of best performers, FDI could increase by about $15 \%$ according to the results obtained with a Poisson model (Table 1). The effect can be larger in countries that have ample room for improvement, such as Chile, Ireland, Israel or Turkey (Figure 5). 
Figure 5. The simplification of regulatory procedures can boost FDI

FDI effect (in per cent) of simplifying regulatory procedures towards the average of the top half of the best performers

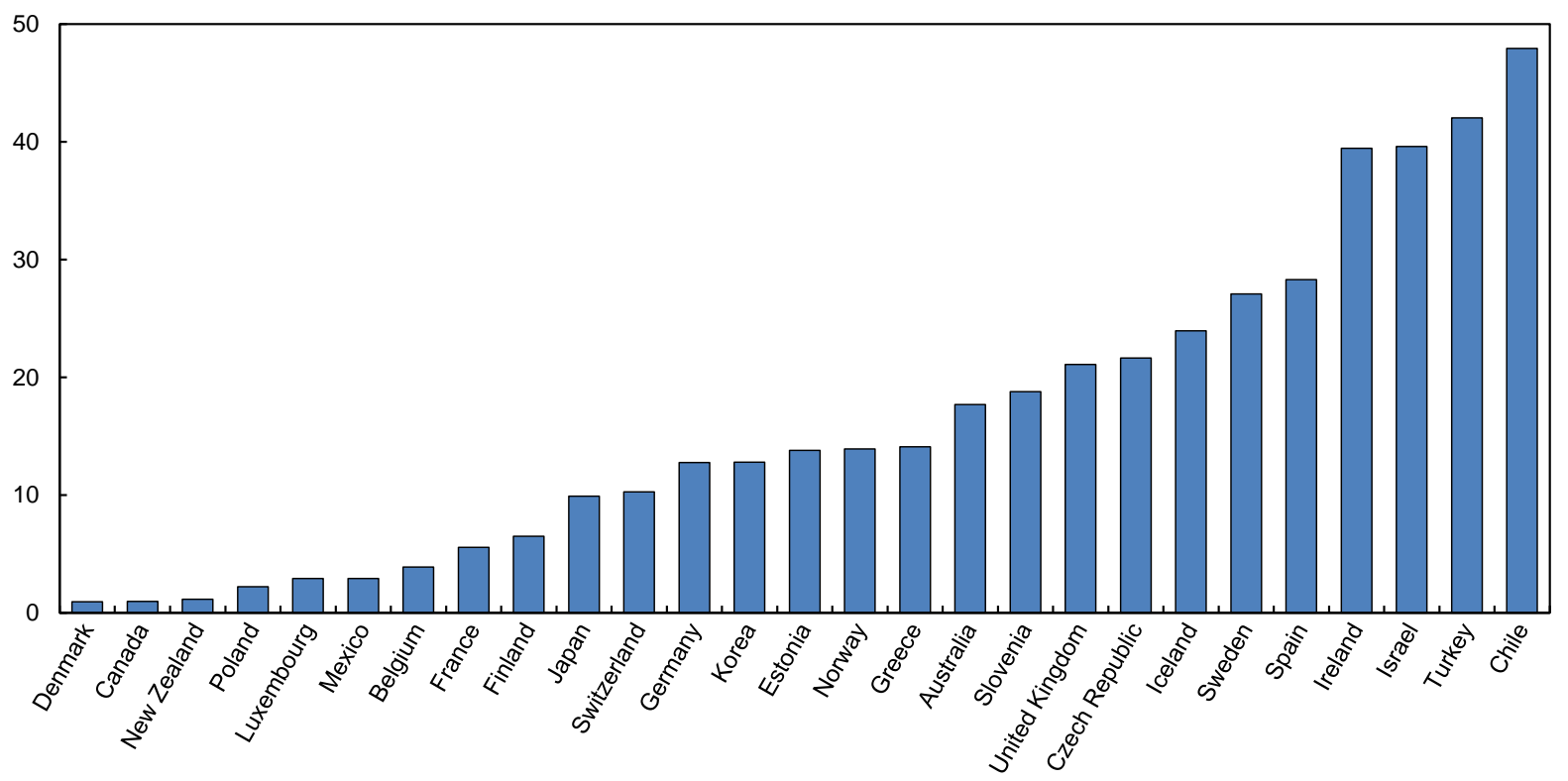

Note: The differences across countries reflect the differences in the complexity of regulatory procedures observed in 2013 . The countries where regulatory procedures are less stringent than the average of the top half are not shown.

Source: OECD calculation based on the average effect of the complexity of regulatory procedures in the host country on FDI stocks as measured in the Poisson models shown in Table 1.

46. These fields of regulation are exceptions as the overall level of product market regulation stringency does not matter much when one takes into account the importance of regulatory heterogeneity (Table 1, Columns 2 and 6). This means that for most regulatory impediments, the effect of regulatory strictness on GDP and on FDI is similar (see Bourlès et al., 2010, among others for evidence of the adverse effect of product market regulation on productivity), so that there is no effect on the FDI-to-GDP ratio. However, there can be an indirect adverse effect on the level on FDI through a negative effect on GDP.

\section{Regulatory protection could increase outward FDI}

47. Regulatory protection of incumbents in their home country can increase profit margins, making firms stronger and hence raise the propensity to invest abroad. There is quite robust evidence that discrimination against foreign suppliers and barriers to trade facilitation increase outward FDI (item "other barriers to trade and investment" in Table A1.6), and some evidence that the regulatory protection of incumbents as measured by the PMR indicator increases outward FDI (item "regulatory protection of incumbents" in Table A1.6). These results need to be interpreted with care as regulatory protections that have adverse effects on firms' competitiveness may reduce FDI less than GDP. This could drive such results, and more research of the effect of regulation protection on FDI would be useful.

\section{EU Single Market and NAFTA}

48. The effect of the Single Market on FDI is positive (Tables 1 and 4). This positive effect is likely to capture the harmonisation of regulations associated with EU accession that go beyond the harmonisation that can be captured with the PMR heterogeneity measure. This Single Market effect is not only present for FDI within the Single Market: FDI from other OECD countries to Single Market countries are boosted as well. This could be because EU countries have signed several trade and FDI agreements with other OECD 
countries, and also because firms outside the Single Market are more likely to establish affiliates in Single Market countries to enter this market. There is also tentative evidence of a positive effect of the euro area beyond the Single Market effect, but this effect is not significant in most specifications. By contrast, the effect of NAFTA within the area is robustly and significantly negative (Tables 1 and 4), potentially reflecting a substitution effect in favour of trade (see Fournier et al., 2015, for evidence of the large positive effect of NAFTA on trade). These results need to be interpreted with care as the OECD sample excludes the closest alternative partners of NAFTA countries. Cuevas et al. (2005) who used a different sample including several Latin American countries find a positive NAFTA effect on FDI flows into Mexico.

\section{Long-run effects could be larger}

49. FDI stocks may adjust to regulatory changes with some delay, so that the current FDI stock reflects both the current and the past state of regulation. Greenfield investments can take time to materialise, and merger and acquisitions may not react immediately as they depend on other factors, such as market conditions. However, firms may also anticipate regulatory changes when the legislative or implementation process is lengthy (e.g. the transposition of EU directives can take several years). Empirically, there is robust evidence that FDI stocks reflect not only current but also the past regulatory level and heterogeneity. Estimates in Table 5 suggest that the long-term effect of a one-fifth regulatory heterogeneity cut could be about $20 \%$. There is also some evidence that the long-run effect of complexity could be about twice larger than the contemporaneous effect. Last, some regressions exhibit a significant positive effect of the euro area on FDI stocks in the long-run. 
Table 5. FDI determinants: Regressions with lags

\begin{tabular}{|c|c|c|c|c|c|c|c|c|}
\hline Estimation method & $\begin{array}{c}(1) \\
\text { Poisson }\end{array}$ & $\begin{array}{c}(2) \\
\text { Poisson } \\
\end{array}$ & $\begin{array}{c}\text { (3) } \\
\text { Poisson }\end{array}$ & $\begin{array}{c}\text { (4) } \\
\text { Poisson }\end{array}$ & $\begin{array}{c}(5) \\
\text { Linear }\end{array}$ & $\begin{array}{c}(6) \\
\text { Linear }\end{array}$ & $\begin{array}{c}(7) \\
\text { Linear }\end{array}$ & $\begin{array}{c}(8) \\
\text { Linear }\end{array}$ \\
\hline $\mathrm{PMR}_{i j t}^{h}$ & $\begin{array}{c}1.00 \\
(0.86)\end{array}$ & $\begin{array}{c}0.83 \\
(0.97)\end{array}$ & $\begin{array}{l}1.93^{\star *} \\
(0.79)\end{array}$ & $\begin{array}{l}-1.12 \\
(0.98)\end{array}$ & $\begin{array}{l}-1.46 \\
(1.10)\end{array}$ & $\begin{array}{l}-2.23^{*} \\
(1.27)\end{array}$ & $\begin{array}{l}-1.06 \\
(1.11)\end{array}$ & $\begin{array}{l}-1.41 \\
(1.05)\end{array}$ \\
\hline $\mathrm{PMR}_{i j t-5}^{h}$ & $\begin{array}{c}-2.73^{\star * *} \\
(0.66)\end{array}$ & $\begin{array}{c}-2.66^{\star * *} \\
(0.74)\end{array}$ & $\begin{array}{c}-2.37^{* * *} \\
(0.60)\end{array}$ & $\begin{array}{c}-1.72^{\star *} \\
(0.74)\end{array}$ & $\begin{array}{c}-2.79^{* * *} \\
(0.82)\end{array}$ & $\begin{array}{l}-2.12^{\star *} \\
(0.91)\end{array}$ & $\begin{array}{c}-2.87^{* * *} \\
(0.83)\end{array}$ & $\begin{array}{c}-2.39^{* * *} \\
(0.80)\end{array}$ \\
\hline $\mathrm{CRP}_{\text {it }}$ & $\begin{array}{l}-0.24^{\star *} \\
(0.097)\end{array}$ & $\begin{array}{c}-0.28^{\star * *} \\
(0.11)\end{array}$ & $\begin{array}{l}-0.29^{* * *} \\
(0.087)\end{array}$ & $\begin{array}{c}-0.36^{* * *} \\
(0.085)\end{array}$ & $\begin{array}{l}0.046 \\
(0.14)\end{array}$ & $\begin{array}{l}0.048 \\
(0.19)\end{array}$ & $\begin{array}{c}0.10 \\
(0.15)\end{array}$ & $\begin{array}{l}0.038 \\
(0.11)\end{array}$ \\
\hline $\mathrm{CRP}_{\text {it }-5}$ & $\begin{array}{l}-0.078^{*} \\
(0.041)\end{array}$ & $\begin{array}{l}-0.12^{*} \\
(0.059)\end{array}$ & $\begin{array}{l}-0.082^{\star} \\
(0.046)\end{array}$ & $\begin{array}{c}-0.22^{\star * \star} \\
(0.052)\end{array}$ & $\begin{array}{c}0.036 \\
(0.085)\end{array}$ & $\begin{array}{c}0.021 \\
(0.097)\end{array}$ & $\begin{array}{c}0.064 \\
(0.090)\end{array}$ & $\begin{array}{l}-0.046 \\
(0.078)\end{array}$ \\
\hline $\mathrm{EEA}_{\text {it }}$ & $\begin{array}{c}-1.01^{* \star *} \\
(0.33)\end{array}$ & $\begin{array}{l}-0.35 \\
(0.48)\end{array}$ & $\begin{array}{c}-1.38^{* \star *} \\
(0.36)\end{array}$ & $\begin{array}{l}0.076 \\
(0.45)\end{array}$ & $\begin{array}{l}-0.026 \\
(0.44)\end{array}$ & $\begin{array}{l}-0.44 \\
(0.70)\end{array}$ & $\begin{array}{c}0.12 \\
(0.45)\end{array}$ & $\begin{array}{c}0.19 \\
(0.39)\end{array}$ \\
\hline $\mathrm{EEA}_{\mathrm{it}-5}$ & $\begin{array}{c}0.29 \\
(0.26)\end{array}$ & $\begin{array}{c}0.20 \\
(0.31)\end{array}$ & $\begin{array}{c}0.44 \\
(0.29)\end{array}$ & $\begin{array}{l}-0.94^{* *} \\
(0.45)\end{array}$ & $\begin{array}{c}0.34 \\
(0.43)\end{array}$ & $\begin{array}{l}-0.17 \\
(0.56)\end{array}$ & $\begin{array}{c}0.49 \\
(0.45)\end{array}$ & $\begin{array}{l}-0.11 \\
(0.39)\end{array}$ \\
\hline $\mathrm{EEA}_{\mathrm{jt}}$ & $\begin{array}{l}1.08^{\star *} \\
(0.51)\end{array}$ & $\begin{array}{l}1.88^{\star *} \\
(0.76)\end{array}$ & $\begin{array}{c}0.87 \\
(0.55)\end{array}$ & $\begin{array}{c}0.58 \\
(0.37)\end{array}$ & $\begin{array}{l}-0.084 \\
(0.49)\end{array}$ & $\begin{array}{c}0.38 \\
(0.69)\end{array}$ & $\begin{array}{l}0.082 \\
(0.53)\end{array}$ & $\begin{array}{l}0.049 \\
(0.40)\end{array}$ \\
\hline $\mathrm{EEA}_{\mathrm{jt}-5}$ & $\begin{array}{c}0.38 \\
(0.59)\end{array}$ & $\begin{array}{l}0.040 \\
(0.61)\end{array}$ & $\begin{array}{l}-0.14 \\
(0.55)\end{array}$ & $\begin{array}{l}-1.01 \\
(0.97)\end{array}$ & $\begin{array}{l}0.83^{*} \\
(0.46)\end{array}$ & $\begin{array}{c}0.73 \\
(0.50)\end{array}$ & $\begin{array}{c}0.71 \\
(0.46)\end{array}$ & $\begin{array}{l}0.85^{\star *} \\
(0.43)\end{array}$ \\
\hline $\mathrm{EEA}_{\mathrm{ijt}}$ & $\begin{array}{l}1.17^{* * *} \\
(0.33)\end{array}$ & $\begin{array}{l}1.23^{* \star *} \\
(0.32)\end{array}$ & $\begin{array}{l}1.45^{\star \star *} \\
(0.33)\end{array}$ & $\begin{array}{c}0.47 \\
(0.49)\end{array}$ & $\begin{array}{l}0.83^{\star *} \\
(0.36)\end{array}$ & $\begin{array}{l}0.83^{* *} \\
(0.37)\end{array}$ & $\begin{array}{l}0.70^{*} \\
(0.37)\end{array}$ & $\begin{array}{l}0.58^{*} \\
(0.32)\end{array}$ \\
\hline $\mathrm{EEA}_{\mathrm{ijt}-5}$ & $\begin{array}{c}-0.78^{\star * *} \\
(0.23)\end{array}$ & $\begin{array}{c}-0.82^{\star * *} \\
(0.23)\end{array}$ & $\begin{array}{c}-0.81^{* * *} \\
(0.23)\end{array}$ & $\begin{array}{l}-0.20 \\
(0.48)\end{array}$ & $\begin{array}{c}-0.94^{* * *} \\
(0.33)\end{array}$ & $\begin{array}{c}-0.95^{\star * *} \\
(0.33)\end{array}$ & $\begin{array}{c}-0.88^{* * *} \\
(0.33)\end{array}$ & $\begin{array}{c}-0.63^{\star \star} \\
(0.31)\end{array}$ \\
\hline $\mathrm{EA}_{\mathrm{ijt}}$ & $\begin{array}{l}0.036 \\
(0.19)\end{array}$ & $\begin{array}{l}-0.17 \\
(0.21)\end{array}$ & $\begin{array}{l}0.060 \\
(0.16)\end{array}$ & $\begin{array}{l}-0.024 \\
(0.19)\end{array}$ & $\begin{array}{c}0.15 \\
(0.22)\end{array}$ & $\begin{array}{l}0.010 \\
(0.24)\end{array}$ & $\begin{array}{c}0.19 \\
(0.22)\end{array}$ & $\begin{array}{l}0.029 \\
(0.21)\end{array}$ \\
\hline $\mathrm{EA}_{\mathrm{ijt}-5}$ & $\begin{array}{l}0.19^{*} \\
(0.10)\end{array}$ & $\begin{array}{c}0.48^{* \star *} \\
(0.16)\end{array}$ & $\begin{array}{l}0.23^{* *} \\
(0.11)\end{array}$ & $\begin{array}{l}-0.026 \\
(0.13)\end{array}$ & $\begin{array}{l}0.061 \\
(0.20)\end{array}$ & $\begin{array}{c}0.27 \\
(0.23)\end{array}$ & $\begin{array}{l}0.087 \\
(0.20)\end{array}$ & $\begin{array}{c}-0.0026 \\
(0.17)\end{array}$ \\
\hline NAFTA $_{i j t}$ & $\begin{array}{c}-1.41^{\star * *} \\
(0.30)\end{array}$ & $\begin{array}{c}-1.33^{\star \star \star} \\
(0.31)\end{array}$ & $\begin{array}{c}-0.95^{\star \star \star} \\
(0.28)\end{array}$ & $\begin{array}{c}-1.17^{* \star *} \\
(0.26)\end{array}$ & $\begin{array}{l}-1.18^{*} \\
(0.64)\end{array}$ & $\begin{array}{l}-1.20^{*} \\
(0.65)\end{array}$ & $\begin{array}{c}-1.40^{* *} \\
(0.67)\end{array}$ & $\begin{array}{l}-0.99 \\
(0.61)\end{array}$ \\
\hline Tariff $_{\text {it }}$ & $\begin{array}{l}-0.32 \\
(0.20)\end{array}$ & & $\begin{array}{l}-0.19 \\
(0.18)\end{array}$ & $\begin{array}{l}-0.027 \\
(0.15)\end{array}$ & $\begin{array}{l}0.48^{\star} \\
(0.29)\end{array}$ & & $\begin{array}{c}0.42 \\
(0.31)\end{array}$ & $\begin{array}{c}0.11 \\
(0.22)\end{array}$ \\
\hline Tariff $_{\text {it }-5}$ & $\begin{array}{l}-0.18 \\
(0.15)\end{array}$ & & $\begin{array}{l}0.018 \\
(0.14)\end{array}$ & $\begin{array}{l}-0.47^{\star \star \star} \\
(0.15)\end{array}$ & $\begin{array}{l}-0.058 \\
(0.19)\end{array}$ & & $\begin{array}{l}-0.17 \\
(0.21)\end{array}$ & $\begin{array}{l}-0.24 \\
(0.16)\end{array}$ \\
\hline Tariff $_{j t}$ & $\begin{array}{l}0.19 \\
(0.12)\end{array}$ & & $\begin{array}{l}0.15 \\
(0.12)\end{array}$ & $\begin{array}{l}0.031 \\
(0.14)\end{array}$ & $\begin{array}{l}-0.17 \\
(0.13)\end{array}$ & & $\begin{array}{l}-0.17 \\
(0.13)\end{array}$ & $\begin{array}{c}-0.30^{\star *} \\
(0.13)\end{array}$ \\
\hline Tariff $_{\mathrm{jt}-5}$ & $\begin{array}{l}0.15 \\
(0.19)\end{array}$ & & $\begin{array}{c}0.20 \\
(0.18)\end{array}$ & $\begin{array}{l}-0.14 \\
(0.19)\end{array}$ & $\begin{array}{l}0.076 \\
(0.16)\end{array}$ & & $\begin{array}{l}0.061 \\
(0.16)\end{array}$ & $\begin{array}{l}-0.069 \\
(0.16)\end{array}$ \\
\hline FDI rest. index $i t$ & $\begin{array}{c}0.31 \\
(0.29)\end{array}$ & & $\begin{array}{l}-0.093 \\
(0.29)\end{array}$ & $\begin{array}{l}0.54^{*} \\
(0.29)\end{array}$ & $\begin{array}{l}0.20 \\
(0.34)\end{array}$ & & $\begin{array}{l}0.35 \\
(0.37)\end{array}$ & $\begin{array}{l}0.41 \\
(0.32)\end{array}$ \\
\hline FDI rest. index $i t-5$ & $\begin{array}{l}-0.32^{* \star *} \\
(0.099)\end{array}$ & & $\begin{array}{l}-0.27^{\star \star *} \\
(0.093)\end{array}$ & $\begin{array}{l}-0.12 \\
(0.11)\end{array}$ & $\begin{array}{l}-0.25^{*} \\
(0.13)\end{array}$ & & $\begin{array}{l}-0.27^{* *} \\
(0.13)\end{array}$ & $\begin{array}{l}-0.15 \\
(0.13)\end{array}$ \\
\hline$N$ & 1417 & 1417 & 1351 & 1705 & 1417 & 1417 & 1351 & 1705 \\
\hline$R^{2}$ & 0.893 & 0.901 & 0.915 & 0.863 & 0.824 & 0.826 & 0.823 & 0.819 \\
\hline Sample & OECD31 & OECD31 & OECD31 & OECD34 & OECD31 & OECD31 & OECD31 & OECD34 \\
\hline $\begin{array}{l}\text { Country and year } \\
\text { fixed effects }\end{array}$ & YES & YES & YES & YES & YES & YES & YES & YES \\
\hline $\begin{array}{l}\text { Controls for PMR } \\
\text { levels in seven } \\
\text { subdomains }\end{array}$ & NO & YES & NO & NO & NO & YES & NO & NO \\
\hline $\begin{array}{l}\text { Controls for size and } \\
\text { factor dissimilarities }\end{array}$ & NO & NO & YES & NO & NO & NO & YES & NO \\
\hline
\end{tabular}

Note: Asterisks $\left({ }^{*},{ }^{* *},{ }^{* *}\right)$ indicate the significance level $(10 \%, 5 \%, 1 \%)$ of the coefficients. i denotes the inward country and $j$ the outward country. Standard errors adjusted for country-pair clusters are in parentheses. Lagged variables are added on top of the baseline regressions shown in Table 1. $\mathrm{CRP}_{\mathrm{it}}=$ Complexity of regulatory procedures, $\mathrm{EEA}_{\mathrm{it}}=$ European Economic Area dummy, $E A_{\mathrm{ijt}}=$ euro area pair dummy, NAFTA $\mathrm{A}_{\mathrm{ij}}=\mathrm{North}$ American Free Trade Agreement pair dummy, $\mathrm{PMR}_{\mathrm{it}}=$ Strictness of employment protection for regular contracts, Tariff $\mathrm{it}_{\mathrm{it}}=\mathrm{PMR}$ indicator of trade tariffs, $\mathrm{PMR}_{\mathrm{it}}=$ Product market regulation overall indicator, $\mathrm{PMR}_{\mathrm{ijt}}^{\mathrm{h}}=$ Product market regulation indicator heterogeneity. Poisson $=$ Poisson pseudomaximum likelihood. OECD31 refers to all OECD countries except Hungary, Luxembourg and the Netherlands that have the highest share of special purpose entities, OEC34 refers to all OECD countries. 


\section{Conclusion}

50. This paper provides robust evidence of the negative effect of the heterogeneity of regulations on FDI. Indeed, this negative effect is found with different indicators: the PMR heterogeneity measure, the Doing Business heterogeneity measure, the dummy capturing the fact that countries share the same legal system and to some extent with the EU Single Market dummy. This suggests a general link between regulatory alignment and FDI, which is likely to hold for many regulatory harmonisation initiatives, including those that could not be included in the empirical analysis. After the numerous FDI facilitation agreements that have reduced explicit barriers to FDI, FDI could be further raised by moves towards common standards and rules to facilitate cross-border activities of firms.

51. In practice, the first-best option to better align regulations is international regulatory cooperation. First, countries can agree on common rules that replace country-specific rules. This can be achieved with International Regulatory Cooperation, as described in OECD (2013). For instance, regulatory co-operation can be promoted by the OECD in each specific area as illustrated by the Chemical Safety case (Sigman, 2013). Regulatory cooperation can also be promoted by a bilateral body, such as the Canada-U.S. Regulatory Cooperation Council (Heynen, 2013). Second, Free Trade Agreements can include the alignment of some regulatory requirements. Third, harmonisation can be achieved by supra-national law, such as the EU regulations.

52. Unilateral suppression of unnecessary regulations can also help to reduce regulatory divergence: each country can suppress unnecessary regulations. Each regulation is indeed likely to induce country specificities and procedures with which local firms are more familiar.

53. Beyond the evidence on the effect of the heterogeneity of product market regulation provided in this paper, other forms of heterogeneity can also create costs for firm. Further research could shine a light on the effect of other forms of heterogeneity, such as the heterogeneity in employment protection legislation, the heterogeneity of environmental policies or the heterogeneity of institutional settings. 
ECO/WKP(2015)86

\section{Bibliography}

Aghion, P., Comin, D., and Howitt, P. (2006), "When Does Domestic Saving Matter for Economic Growth?”, NBER Working Papers, No. 12275.

Alfaro, L, A. Chanda, S. Kalemli-Ozcan and S. Sayek (2004), "FDI and Economic Growth: the Role of Local Financial Markets", Journal of International Economics, Vol. 64, No. 1, pp. 89-112.

Anderson, J. E. and Van Wincoop (2003), "Gravity with Gravitas: A Solution to the Border Puzzle", American Economic Review, Vol. 93, pp. 170-192.

Andrews, D. and C. Criscuolo (2013), "Knowledge-based Capital, Innovation and Resource Allocation", OECD Economics Department Working Papers, No. 1046, OECD Publishing.

Araújo, S. (2011), "Has Deregulation Increased Investment in Infrastructure? Firm-Level Evidence from OECD Countries", OECD Economics Department Working Papers, No. 892, OECD Publishing.

Autor, D.H., W.R. Kerr and A.D. Kugler (2007), "Do Employment Protections Reduce Productivity? Evidence from US States", Economic Journal, 117, F189-F217.

Balasubramanyam, V. N., M. Salisu and D. Sapsford (1996), "Foreign Direct Investment and Growth in EP and IS Countries", The Economic Journal, Vol. 106, No. 434, pp. 92-105.

Balasubramanyam, V. N., M. Salisu and D. Sapsford (1999), "Foreign Direct Investment as an Engine of Growth", The Journal of International Trade \& Economic Development: An International and Comparative Review, Volume 8, No. 1, pp. 27-40.

Barrell, R. and N. Pain (1997), "Foreign Direct Investment, Technological Change and Economic Growth within Europe", Economic Journal, Vol. 107, No. 445, pp. 1770-1786.

Barro, R., J. and J., W. Lee (2013), "A New Data Set of Educational Attainment in the World, 19502010”, Journal of Development Economics, Vol. 104, pp. 184-198.

Bassanini, A., L. Nunziata and D. Venn (2009), "Job Protection Legislation and Productivity Growth in OECD Countries”, Economic Policy, Vol. 24, pp. 349-402.

Bénassy-Quéré, A., M. Coupet and T. Mayer (2007), "Institutional Determinants of Foreign Direct Investment”, World Economy, Vol 30, No. 5, pp.764-782.

Blanchard, O. and F. Giavazzi (2003), "Macroeconomic Effects of Regulation and Deregulation in Goods and Labor Markets", The Quarterly Journal of Economics, Vol. 118, No. 3, pp. 879-907.

Bourlès, R. et al. (2010), "Do Product Market Regulations in Upstream Sectors Curb Productivity Growth? Panel Data Evidence for OECD Countries", OECD Economics Department Working Papers, No. 791, OECD Publishing. 
Busse, M. and J. L. Groizard (2008), "Foreign Direct Investment, Regulations and Growth", The World Economy, Vol. 31, No. 7, pp. 861-886.

Cuevas, A., M. Messmacher and A. Werner (2005), "Foreign Direct Investment in Mexico since the Approval of NAFTA", World Bank Economic Review, World Bank Group, Vol. 19, No. 3, pp. 473488.

Davidson, W. H. (1980), “The Location of Foreign Direct Investment Activity: Country Characteristics and Experience Effects”, Journal of International Business Studies, Vol. 11, No. 2, pp. 9-22.

de Sousa, J. and J. Lochard (2011), "Does the Single Currency Affect Foreign Direct Investment?", The Scandinavian Journal of Economics, Vol. 113, No. 3, pp. 553-578.

Djankov, S., C. McLiesh and A. Shleifer (2007), "Private Credit in 129 Countries", Journal of Financial Economics, Elsevier, 84(2): 299-329.

Ethier and Markusen (1996), "Multinational Firms, Technology Diffusion and Trade", Vol. 41, No. 1-2, pp. 1-28.

Eaton, J. and A. Tamura (1994), "Bilateralism and Regionalism in Japanese and U.S. Trade and Direct Foreign Investment Patterns", Journal of the Japanese and International Economies, Vol. 8, pp. 478-510.

Fournier, J.-M. (2014), "Reinvigorating the EU Single Market", OECD Economics Department Working Papers, No. 1159, OECD Publishing.

Fournier, J.-M. (2015), “The Heterogeneity of Product Market Regulations”, OECD Economics Department Working Papers, No. 1182, OECD Publishing.

Fournier, J.-M. et al. (2015), "Implicit Regulatory Barriers in the EU Single Market: New Empirical Evidence from Gravity Models”, OECD Economics Department Working Papers, No. 1181, OECD Publishing.

Golub, S. et al. (2003), “The Influence of Policies on Trade and Foreign Direct Investment”, OECD Economic Studies, No. 36, pp. 7-83.

Habib, M. and L. Zurawicki (2002), "Corruption and Foreign Direct Investment”, Journal of International Business Studies, Vol. 33, No. 2, pp. 291-307.

Haltiwanger, J., S. Scarpetta and H. Schweiger (2006), “Assessing Job Flows across Countries: The Role of Industry, Firm Size and Regulations”, IZA Discussion Paper, No. 2450, Bonn.

Head, K. and J. Ries (2008), "FDI as an Outcome of the Market for Corporate Control: Theory and Evidence", Journal of International Economics, Vol. 74 (1), pp. 2-20.

Heynen, J. (2013), “The Canada-U.S. Regulatory Cooperation Council”, in International Regulatory Cooperation: Case Studies, Vol. 2, OECD Publishing.

Javorcik, B. S. (2004), "Does Foreign Direct Investment Increase the Productivity of Domestic Firms? In Search of Spillovers through Backward Linkages", The American Economic Review, Vol. 94, No. 3, pp. 605-627. 
Kalinova, B., A. Palerm and S. Thomsen (2010), "OECD's FDI Restrictiveness Index: 2010 Update", OECD Working Papers on International Investment, No. 2010/3.

Keller, W. and A. Levinson (2002), "Pollution Abatement Costs and Foreign Direct Investment Inflows to U.S. States", The Review of Economics and Statistics, Vol. 84, No. 4, pp. 691-703.

Koske, I. et al. (2015), "The 2013 Up-date of the OECD Product Market Regulation Indicators: Policy Insights for OECD and non-OECD Countries", OECD Economics Department Working Paper, No. 1200, OECD Publishing.

Kox, H. and A. Lejour (2006), "The Effects of the Services Directive on Intra-EU Trade and FDI", Revue économique, Presses de Sciences Po, Vol. 57, pp. 747 à 769.

Kox, H., A. Lejour and R. Montizaan (2004), "The Free Movement of Services within the EU”, $C P B$ Document 69.

Levine, R., C. Lin and B. Shen (2015), "Cross-Border Acquisitions and Labor Regulations, NBER Working Papers, No. 21245.

Lesher, M. and S. Miroudot (2008), "FDI Spillovers and their Interrelationships with Trade", OECD Trade Policy Papers, No. 80, OECD Publishing.

Markusen (1997), “Trade versus Investment Liberalisation”, NBER Working Papers, No. 6231.

Markusen, Venables, Eby-Konan and Zhang, K. H. (1996), “A Unified Treatment of Horizontal Direct Investment, Vertical Direct Investment, and the Pattern of Trade in Goods and Services", NBER Working Paper No. 5696.

Nordås, H. K. and H. Kox (2009), "Quantifying Regulatory Barriers to Services Trade”, OECD Trade Policy Papers, No. 85, OECD Publishing, Paris.

OECD (1996), OECD Benchmark Definition of Foreign Direct Investment, Third Edition, OECD Publishing, Paris.

OECD (2007), Tax Effects on Foreign Direct Investment, OECD Publishing, Paris.

OECD (2008), OECD Benchmark Definition of Foreign Direct Investment, Fourth Edition, OECD Publishing, Paris.

Okawa, Y. and E. van Wincoop (2012), "Gravity in International Finance", Journal of International Economics, Vol. 87, No. 2, pp. 205-215.

Palumbo, G., G. Giupponi, L. Nunziata and J.S. Mora-Sanguinetti (2013), "The Economics of Civil Justice: New Cross-country Data and Empirics", OECD Economics Department Working Papers, No. 1060, OECD Publishing.

Portes, R., Rey, H. (2005), “The Determinants of Cross-border Equity Flows”, Journal of International Economics, Vol. 65, No. 2, pp. 269-296.

Rowland, P. and L. Tesar (2004), "Multinationals and the Gains from International Diversification", Review of Economic Dynamics, Vol. 7, No. 4, pp. 789-826. 


\section{ECO/WKP(2015)86}

Santos Silva, J.M.C. and S. Tenreyro (2006), "The Log of Gravity", The Review of Economics and Statistics, Vol. 88(4), pp. 641-658.

Sigman, R. (2013), "Chemical Safety", in International Regulatory Co-operation: Case Studies, Vol. 1, OECD Publishing.

Stein, E. and C. Daude (2007), "Longitude Matters: Time Zones and the Location of Foreign Direct Investment", Journal of International Economics, Vol. 71, No. 1, pp. 96-112. 
ECO/WKP(2015)86

\section{APPENDIX}

Table A1.1. The effect of factor dissimilarity and size similarity

\begin{tabular}{|c|c|c|c|c|c|c|}
\hline Estimation method & $\begin{array}{c}(1) \\
\text { Poisson }\end{array}$ & $\begin{array}{c}(2) \\
\text { Linear }\end{array}$ & $\begin{array}{c}(3) \\
\text { Poisson }\end{array}$ & $\begin{array}{c}(4) \\
\text { Linear }\end{array}$ & $\begin{array}{c}(5) \\
\text { Poisson }\end{array}$ & $\begin{array}{c}(6) \\
\text { Linear }\end{array}$ \\
\hline $\mathrm{HCD}_{\mathrm{ijt}}$ & $\begin{array}{l}0.63 \\
(0.39)\end{array}$ & $\begin{array}{c}0.28 \\
(0.49)\end{array}$ & $\begin{array}{c}0.47 \\
(0.40)\end{array}$ & $\begin{array}{c}0.15 \\
(0.50)\end{array}$ & $\begin{array}{c}0.39 \\
(0.35)\end{array}$ & $\begin{array}{l}0.025 \\
(0.48)\end{array}$ \\
\hline $\mathrm{FD}_{\mathrm{ijt}}$ & $\begin{array}{c}-0.64^{* * *} \\
(0.15)\end{array}$ & $\begin{array}{c}0.19 \\
(0.11)\end{array}$ & $\begin{array}{c}-0.62^{* \star *} \\
(0.15)\end{array}$ & $\begin{array}{c}0.19 \\
(0.12)\end{array}$ & $\begin{array}{c}-0.58^{\star * *} \\
(0.13)\end{array}$ & $\begin{array}{l}0.16 \\
(0.10)\end{array}$ \\
\hline $\mathrm{SS}_{\mathrm{ijt}}$ & $\begin{array}{l}0.43^{* * *} \\
(0.058)\end{array}$ & $\begin{array}{l}0.31^{* * *} \\
(0.069)\end{array}$ & $\begin{array}{l}0.41^{* * *} \\
(0.059)\end{array}$ & $\begin{array}{l}0.29^{\star \star \star} \\
(0.068)\end{array}$ & $\begin{array}{l}0.47^{* * *} \\
(0.071)\end{array}$ & $\begin{array}{l}0.37^{\star * *} \\
(0.068)\end{array}$ \\
\hline $\begin{array}{l}\text { Columns showing the } \\
\text { other regression results }\end{array}$ & $\begin{array}{l}\text { Table 1; } \\
\text { column } 4\end{array}$ & $\begin{array}{l}\text { Table 1; } \\
\text { column } 9\end{array}$ & $\begin{array}{l}\text { Table 3; } \\
\text { column } 4\end{array}$ & $\begin{array}{l}\text { Table 3; } \\
\text { column } 9\end{array}$ & $\begin{array}{l}\text { Table 4; } \\
\text { column } 4\end{array}$ & $\begin{array}{l}\text { Table 4; } \\
\text { column } 9\end{array}$ \\
\hline
\end{tabular}

Note: Asterisks $\left({ }^{*},{ }^{* *},{ }^{* * *}\right)$ indicate the significance level $(10 \%, 5 \%, 1 \%)$ of the coefficients. i denotes the inward country and $j$ the outward country. Standard errors adjusted for country-pair clusters are in parentheses. $\mathrm{HCD}_{\mathrm{ij}}=$ human capital dissimilarity (absolute difference in the average number of years of schooling), $\mathrm{FD}_{\mathrm{ij}}=$ factor dissimilarity (difference in GDP per capita used as a proxy for the difference in the stock of capital), $\mathrm{SS}_{\mathrm{ijt}}=$ size similarity. These variables are added on top of the baseline regressions shown in other tables. Poisson = Poisson pseudo-maximum likelihood. 


\section{ECO/WKP(2015)86}

Table A1.2. FDI determinants: Doing business heterogeneity indicator results

\begin{tabular}{|c|c|c|c|c|c|c|c|c|}
\hline Estimation method & $\begin{array}{c}(1) \\
\text { Poisson }\end{array}$ & $\begin{array}{c}(2) \\
\text { Poisson }\end{array}$ & $\begin{array}{c}\text { (3) } \\
\text { Poisson }\end{array}$ & $\begin{array}{c}\text { (4) } \\
\text { Poisson }\end{array}$ & $\begin{array}{c}(5) \\
\text { Linear }\end{array}$ & $\begin{array}{c}(6) \\
L i n e a r\end{array}$ & $\begin{array}{c}(7) \\
\text { Linear }\end{array}$ & $\begin{array}{c}(8) \\
\text { Linear }\end{array}$ \\
\hline $\ln \left(\mathrm{Y}_{\mathrm{it}}\right)$ & $\begin{array}{c}0.75 \\
(0.52)\end{array}$ & $\begin{array}{c}-0.055 \\
(0.54)\end{array}$ & $\begin{array}{c}0.23 \\
(0.61)\end{array}$ & $\begin{array}{l}0.84^{*} \\
(0.48)\end{array}$ & $\begin{array}{l}1.80^{* \star *} \\
(0.64)\end{array}$ & $\begin{array}{c}1.83^{\star * *} \\
(0.65)\end{array}$ & $\begin{array}{l}1.96^{\star * *} \\
(0.68)\end{array}$ & $\begin{array}{c}1.71^{* \star *} \\
(0.59)\end{array}$ \\
\hline $\ln \left(Y_{j t}\right)$ & $\begin{array}{l}0.057 \\
(0.51)\end{array}$ & $\begin{array}{l}-0.84 \\
(0.60)\end{array}$ & $\begin{array}{c}0.95 \\
(0.94)\end{array}$ & $\begin{array}{c}0.29 \\
(0.50)\end{array}$ & $\begin{array}{c}0.42 \\
(0.58)\end{array}$ & $\begin{array}{c}0.50 \\
(0.58)\end{array}$ & $\begin{array}{c}0.45 \\
(0.62)\end{array}$ & $\begin{array}{c}0.24 \\
(0.58)\end{array}$ \\
\hline $\ln \left(\right.$ dist $\left._{\mathrm{ij}}\right)$ & $\begin{array}{c}-0.58^{* * *} \\
(0.12)\end{array}$ & $\begin{array}{c}-0.49^{* * *} \\
(0.11)\end{array}$ & $\begin{array}{l}-0.64^{\star \star \star *} \\
(0.089)\end{array}$ & $\begin{array}{c}-0.50^{* \star *} \\
(0.11)\end{array}$ & $\begin{array}{c}-0.99^{* * *} \\
(0.15)\end{array}$ & $\begin{array}{c}-0.98^{\star \star * *} \\
(0.15)\end{array}$ & $\begin{array}{c}-0.99^{* * *} \\
(0.15)\end{array}$ & $\begin{array}{c}-0.92^{* * *} \\
(0.14)\end{array}$ \\
\hline Contiguity $_{\mathrm{ij}}$ & $\begin{array}{c}0.12 \\
(0.15)\end{array}$ & $\begin{array}{c}0.26^{*} \\
(0.14)\end{array}$ & $\begin{array}{c}0.11 \\
(0.13)\end{array}$ & $\begin{array}{c}0.24 \\
(0.16)\end{array}$ & $\begin{array}{l}-0.023 \\
(0.27)\end{array}$ & $\begin{array}{l}-0.048 \\
(0.26)\end{array}$ & $\begin{array}{l}-0.11 \\
(0.27)\end{array}$ & $\begin{array}{l}0.064 \\
(0.25)\end{array}$ \\
\hline Same legal system $_{i j}$ & $\begin{array}{c}0.50^{\star \star \star *} \\
(0.10)\end{array}$ & $\begin{array}{l}0.40^{\star \star \star} \\
(0.091)\end{array}$ & $\begin{array}{l}0.38^{\star \star \star *} \\
(0.096)\end{array}$ & $\begin{array}{c}0.33^{\star \star \star} \\
(0.10)\end{array}$ & $\begin{array}{c}0.68^{* * *} \\
(0.13)\end{array}$ & $\begin{array}{c}0.68^{* \star *} \\
(0.13)\end{array}$ & $\begin{array}{c}0.71^{* \star \star} \\
(0.13)\end{array}$ & $\begin{array}{c}0.71^{* \star \star} \\
(0.12)\end{array}$ \\
\hline Common language $_{i j}$ & $\begin{array}{l}0.082 \\
(0.15)\end{array}$ & $\begin{array}{c}0.14 \\
(0.13)\end{array}$ & $\begin{array}{c}0.38^{\star \star \star} \\
(0.15)\end{array}$ & $\begin{array}{c}0.14 \\
(0.14)\end{array}$ & $\begin{array}{l}0.42^{*} \\
(0.24)\end{array}$ & $\begin{array}{l}0.45^{\star} \\
(0.24)\end{array}$ & $\begin{array}{l}0.65^{\star \star} \\
(0.25)\end{array}$ & $\begin{array}{l}0.43^{\star} \\
(0.23)\end{array}$ \\
\hline $\ln \left(\right.$ Remoteness $\left._{\mathrm{it}}\right)$ & $\begin{array}{l}-2.03^{*} \\
(1.20)\end{array}$ & $\begin{array}{c}-4.04^{* * *} \\
(1.34)\end{array}$ & $\begin{array}{l}-0.80 \\
(2.50)\end{array}$ & $\begin{array}{l}-1.85 \\
(1.14)\end{array}$ & $\begin{array}{l}-0.87 \\
(1.82)\end{array}$ & $\begin{array}{l}-0.82 \\
(1.82)\end{array}$ & $\begin{array}{l}-1.22 \\
(2.14)\end{array}$ & $\begin{array}{l}-1.11 \\
(1.70)\end{array}$ \\
\hline $\ln \left(\right.$ Remoteness $\left._{\mathrm{jt}}\right)$ & $\begin{array}{l}-0.075 \\
(1.33)\end{array}$ & $\begin{array}{l}-1.38 \\
(1.43)\end{array}$ & $\begin{array}{l}-0.56 \\
(3.45)\end{array}$ & $\begin{array}{l}-0.11 \\
(1.28)\end{array}$ & $\begin{array}{l}-0.63 \\
(2.24)\end{array}$ & $\begin{array}{l}-0.76 \\
(2.23)\end{array}$ & $\begin{array}{l}-0.98 \\
(2.65)\end{array}$ & $\begin{array}{l}-0.66 \\
(2.03)\end{array}$ \\
\hline $\ln \left(\right.$ Eff. exch. rate $\left._{i t}\right)$ & $\begin{array}{l}-0.11 \\
(0.76)\end{array}$ & $\begin{array}{c}0.20 \\
(0.75)\end{array}$ & $\begin{array}{c}-0.28 \\
(1.13)\end{array}$ & $\begin{array}{l}-0.20 \\
(0.70)\end{array}$ & $\begin{array}{l}-1.63 \\
(1.02)\end{array}$ & $\begin{array}{l}-1.66 \\
(1.03)\end{array}$ & $\begin{array}{l}-1.85 \\
(1.18)\end{array}$ & $\begin{array}{l}-1.44 \\
(0.93)\end{array}$ \\
\hline $\ln \left(\right.$ Eff. exch. rate $\left._{j \mathrm{t}}\right)$ & $\begin{array}{l}1.31^{*} \\
(0.70)\end{array}$ & $\begin{array}{c}1.99^{\star \star \star} \\
(0.76)\end{array}$ & $\begin{array}{c}0.35 \\
(1.72)\end{array}$ & $\begin{array}{c}1.10 \\
(0.69)\end{array}$ & $\begin{array}{c}0.59 \\
(0.98)\end{array}$ & $\begin{array}{c}0.59 \\
(0.98)\end{array}$ & $\begin{array}{c}0.77 \\
(1.12)\end{array}$ & $\begin{array}{l}1.00 \\
(0.96)\end{array}$ \\
\hline $\mathrm{EEA}_{\mathrm{it}}$ & $\begin{array}{l}-0.13 \\
(0.18)\end{array}$ & $\begin{array}{c}-0.41^{* *} \\
(0.19)\end{array}$ & $\begin{array}{l}-0.16 \\
(0.23)\end{array}$ & $\begin{array}{l}-0.13 \\
(0.17)\end{array}$ & $\begin{array}{l}0.067 \\
(0.19)\end{array}$ & $\begin{array}{l}0.045 \\
(0.19)\end{array}$ & $\begin{array}{c}0.14 \\
(0.19)\end{array}$ & $\begin{array}{c}0.0087 \\
(0.16)\end{array}$ \\
\hline $\mathrm{EEA}_{\mathrm{jt}}$ & $\begin{array}{c}0.39 \\
(0.33)\end{array}$ & $\begin{array}{c}0.30 \\
(0.28)\end{array}$ & $\begin{array}{c}0.17 \\
(0.25)\end{array}$ & $\begin{array}{l}-0.025 \\
(0.17)\end{array}$ & $\begin{array}{l}-0.13 \\
(0.21)\end{array}$ & $\begin{array}{l}-0.17 \\
(0.21)\end{array}$ & $\begin{array}{l}-0.079 \\
(0.23)\end{array}$ & $\begin{array}{l}0.011 \\
(0.18)\end{array}$ \\
\hline$E E A_{i j t}$ & $\begin{array}{c}0.46 \\
(0.28)\end{array}$ & $\begin{array}{l}0.68^{* *} \\
(0.27)\end{array}$ & $\begin{array}{c}0.27 \\
(0.30)\end{array}$ & $\begin{array}{c}0.39 \\
(0.25)\end{array}$ & $\begin{array}{c}0.22 \\
(0.24)\end{array}$ & $\begin{array}{c}0.24 \\
(0.24)\end{array}$ & $\begin{array}{c}0.15 \\
(0.25)\end{array}$ & $\begin{array}{c}0.19 \\
(0.22)\end{array}$ \\
\hline $\mathrm{EA}_{\mathrm{ijt}}$ & $\begin{array}{c}0.21 \\
(0.17)\end{array}$ & $\begin{array}{c}0.10 \\
(0.15)\end{array}$ & $\begin{array}{c}0.12 \\
(0.16)\end{array}$ & $\begin{array}{c}0.12 \\
(0.17)\end{array}$ & $\begin{array}{l}0.093 \\
(0.21)\end{array}$ & $\begin{array}{c}0.16 \\
(0.20)\end{array}$ & $\begin{array}{c}0.18 \\
(0.21)\end{array}$ & $\begin{array}{l}-0.034 \\
(0.19)\end{array}$ \\
\hline NAFTA $_{i j}$ & $\begin{array}{c}-1.00^{* * *} \\
(0.33)\end{array}$ & $\begin{array}{c}-0.76^{\star * *} \\
(0.30)\end{array}$ & $\begin{array}{c}-0.79^{* * *} \\
(0.27)\end{array}$ & $\begin{array}{c}-0.74^{\text {***}} \\
(0.28)\end{array}$ & $\begin{array}{l}-0.28 \\
(0.77)\end{array}$ & $\begin{array}{l}-0.45 \\
(0.79)\end{array}$ & $\begin{array}{c}0.38 \\
(0.74)\end{array}$ & $\begin{array}{l}-0.19 \\
(0.78)\end{array}$ \\
\hline$E P L_{\text {it }}$ & $\begin{array}{l}-0.21 \\
(0.25)\end{array}$ & $\begin{array}{l}-0.33 \\
(0.25)\end{array}$ & $\begin{array}{l}-0.28 \\
(0.39)\end{array}$ & $\begin{array}{r}-0.075 \\
(0.33)\end{array}$ & $\begin{array}{l}-0.31 \\
(0.36)\end{array}$ & $\begin{array}{l}-0.28 \\
(0.36)\end{array}$ & $\begin{array}{l}-0.22 \\
(0.39)\end{array}$ & $\begin{array}{l}-0.36 \\
(0.34)\end{array}$ \\
\hline$E P L_{j t}$ & $\begin{array}{l}-0.50^{* *} \\
(0.21)\end{array}$ & $\begin{array}{c}-0.70^{\star * *} \\
(0.24)\end{array}$ & $\begin{array}{l}-0.71 \\
(0.49)\end{array}$ & $\begin{array}{l}-0.52^{* *} \\
(0.21)\end{array}$ & $\begin{array}{l}-0.44 \\
(0.37)\end{array}$ & $\begin{array}{l}-0.40 \\
(0.37)\end{array}$ & $\begin{array}{l}-0.51 \\
(0.41)\end{array}$ & $\begin{array}{l}-0.39 \\
(0.35)\end{array}$ \\
\hline $\mathrm{ETCR}_{\text {it }}$ & & & $\begin{array}{c}0.11 \\
(0.20)\end{array}$ & & & & $\begin{array}{l}0.35^{\star} \\
(0.19)\end{array}$ & \\
\hline ETC $_{\mathrm{jt}}$ & & & $\begin{array}{l}0.0037 \\
(0.19)\end{array}$ & & & & $\begin{array}{l}-0.038 \\
(0.19)\end{array}$ & \\
\hline $\mathrm{DB}_{\mathrm{ijt}}^{\mathrm{h}}$ & $\begin{array}{l}-0.60^{* *} \\
(0.29)\end{array}$ & $\begin{array}{l}-0.31 \\
(0.25)\end{array}$ & $\begin{array}{l}-0.34 \\
(0.29)\end{array}$ & $\begin{array}{c}-0.84^{* * *} \\
(0.28)\end{array}$ & $\begin{array}{c}-1.52^{* * *} \\
(0.52)\end{array}$ & $\begin{array}{c}-1.68^{\star * *} \\
(0.47)\end{array}$ & $\begin{array}{c}-1.77^{\star * *} \\
(0.50) \\
\end{array}$ & $\begin{array}{c}-1.37^{* * *} \\
(0.47)\end{array}$ \\
\hline$N$ & 2378 & 2378 & 2195 & 2845 & 2378 & 2378 & 2195 & 2845 \\
\hline$R^{2}$ & 0.927 & 0.939 & 0.864 & 0.914 & 0.844 & 0.848 & 0.837 & 0.844 \\
\hline Sample & OECD31 & OECD31 & OECD31 & OECD34 & OECD31 & OECD31 & OECD31 & OECD34 \\
\hline $\begin{array}{l}\text { Country and year } \\
\text { fixed effects }\end{array}$ & YES & YES & YES & YES & YES & YES & YES & YES \\
\hline $\begin{array}{l}\text { Controls for size and } \\
\text { factor dissimilarities }\end{array}$ & NO & YES & YES & NO & NO & YES & YES & NO \\
\hline
\end{tabular}

Note: Asterisks $\left({ }^{*},{ }^{* *},{ }^{* \star *}\right)$ indicate the significance level $(10 \%, 5 \%, 1 \%)$ of the coefficients. i denotes the inward country and $j$ the outward country. Standard errors adjusted for country-pair clusters are in parentheses. $Y_{\text {it }}=$ nominal GDP in US Dollars, Dist $t_{i j}=$

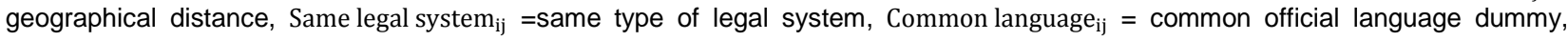

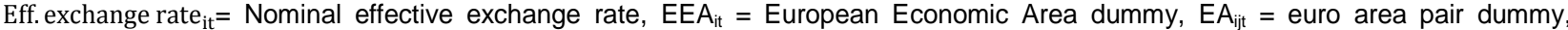
$\mathrm{NAFTA}_{\mathrm{ij}}=$ North American Free Trade Agreement pair dummy, EPL $\mathrm{it}_{\mathrm{it}}=$ Strictness of employment protection for regular contracts, $\mathrm{ETCR}_{\mathrm{it}}=$ Electricity, Transport and Communication Regulation indicator, $\mathrm{DB}_{\mathrm{ijt}}^{\mathrm{h}}=$ Doing Business indicator heterogeneity. Poisson $=$ Poisson pseudo-maximum likelihood. OECD31 refers to all OECD countries except Hungary, Luxembourg and the Netherlands that have the highest shares of Special Purpose entities, OEC34 refers to all OECD countries. 
ECO/WKP(2015)86

Table A1.3. FDI determinants: Robustness checks with tax rates

\begin{tabular}{|c|c|c|c|c|c|c|c|c|c|c|}
\hline Estimation method & $\begin{array}{c}(1) \\
\text { Poisson }\end{array}$ & $\begin{array}{c}(2) \\
\text { Poisson } \\
\end{array}$ & $\begin{array}{c}(3) \\
\text { Poisson }\end{array}$ & $\begin{array}{c}(4) \\
\text { Poisson }\end{array}$ & $\begin{array}{c}(5) \\
\text { Poisson }\end{array}$ & $\begin{array}{c}(6) \\
\text { Linear }\end{array}$ & $\begin{array}{c}(7) \\
\text { Linear }\end{array}$ & $\begin{array}{c}(8) \\
\text { Linear }\end{array}$ & $\begin{array}{c}(9) \\
\text { Linear }\end{array}$ & $\begin{array}{c}(10) \\
\text { Linear }\end{array}$ \\
\hline $\mathrm{PMR}_{i t}$ & & $\begin{array}{l}0.078 \\
(0.21)\end{array}$ & & & & & $\begin{array}{c}0.85^{\star * *} \\
(0.30)\end{array}$ & & & \\
\hline $\mathrm{PMR}_{j t}$ & & $\begin{array}{c}0.25 \\
(0.19)\end{array}$ & & & & & $\begin{array}{l}-0.28 \\
(0.26)\end{array}$ & & & \\
\hline $\mathrm{PMR}_{i j t}^{h}$ & $\begin{array}{c}-1.64^{* * *} \\
(0.63)\end{array}$ & $\begin{array}{c}-1.79^{* * *} \\
(0.66)\end{array}$ & $\begin{array}{c}-1.74^{* *} \\
(0.75)\end{array}$ & $\begin{array}{l}-0.62 \\
(0.58)\end{array}$ & $\begin{array}{c}-2.41^{* * *} \\
(0.63)\end{array}$ & $\begin{array}{c}-2.83^{* * *} \\
(0.83)\end{array}$ & $\begin{array}{c}-2.92^{* * *} \\
(0.85)\end{array}$ & $\begin{array}{c}-3.20^{\star \star *} \\
(0.87)\end{array}$ & $\begin{array}{c}-2.54^{* \star *} \\
(0.85)\end{array}$ & $\begin{array}{c}-2.39^{\star \star *} \\
(0.77)\end{array}$ \\
\hline Tax wedge single $i t$ & $\begin{array}{l}0.042^{* *} \\
(0.020)\end{array}$ & $\begin{array}{l}0.042^{* *} \\
(0.020)\end{array}$ & $\begin{array}{c}0.044^{* * *} \\
(0.016)\end{array}$ & $\begin{array}{l}0.034^{*} \\
(0.020)\end{array}$ & $\begin{array}{c}-0.11^{* * *} \\
(0.034)\end{array}$ & $\begin{array}{c}0.023 \\
(0.026)\end{array}$ & $\begin{array}{c}0.029 \\
(0.026)\end{array}$ & $\begin{array}{c}0.035 \\
(0.027)\end{array}$ & $\begin{array}{c}0.014 \\
(0.027)\end{array}$ & $\begin{array}{l}-0.019 \\
(0.025)\end{array}$ \\
\hline Tax wedge single ${ }_{j t}$ & $\begin{array}{l}0.046^{*} \\
(0.026)\end{array}$ & $\begin{array}{c}0.038 \\
(0.026)\end{array}$ & $\begin{array}{c}0.014 \\
(0.026)\end{array}$ & $\begin{array}{c}0.026 \\
(0.023)\end{array}$ & $\begin{array}{c}0.044 \\
(0.040)\end{array}$ & $\begin{array}{c}0.030 \\
(0.028)\end{array}$ & $\begin{array}{c}0.026 \\
(0.028)\end{array}$ & $\begin{array}{c}0.024 \\
(0.029)\end{array}$ & $\begin{array}{c}0.043 \\
(0.028)\end{array}$ & $\begin{array}{c}0.025 \\
(0.026)\end{array}$ \\
\hline Tax wedge couple it $_{\text {it }}$ & $\begin{array}{c}0.011 \\
(0.011)\end{array}$ & $\begin{array}{c}0.018 \\
(0.013)\end{array}$ & $\begin{array}{l}0.020^{*} \\
(0.012)\end{array}$ & $\begin{array}{c}0.011 \\
(0.011)\end{array}$ & $\begin{array}{c}0.079^{* * *} \\
(0.023)\end{array}$ & $\begin{array}{l}0.043^{* *} \\
(0.017)\end{array}$ & $\begin{array}{c}0.058^{\star \star *} \\
(0.018)\end{array}$ & $\begin{array}{c}0.056^{\star \star \star} \\
(0.018)\end{array}$ & $\begin{array}{l}0.044^{* *} \\
(0.017)\end{array}$ & $\begin{array}{c}0.049^{* * *} \\
(0.015)\end{array}$ \\
\hline Tax wedge couple $j t$ & $\begin{array}{l}-0.0026 \\
(0.016)\end{array}$ & $\begin{array}{l}0.0040 \\
(0.017)\end{array}$ & $\begin{array}{c}0.014 \\
(0.018)\end{array}$ & $\begin{array}{l}0.0031 \\
(0.017)\end{array}$ & $\begin{array}{l}0.0032 \\
(0.027)\end{array}$ & $\begin{array}{l}-0.016 \\
(0.020)\end{array}$ & $\begin{array}{c}-0.017 \\
(0.020)\end{array}$ & $\begin{array}{c}-0.012 \\
(0.021)\end{array}$ & $\begin{array}{l}-0.020 \\
(0.020)\end{array}$ & $\begin{array}{c}-0.015 \\
(0.017)\end{array}$ \\
\hline $\begin{array}{l}\text { Effective Average } \\
\text { Corporate tax rate }_{i t}\end{array}$ & $\begin{array}{c}-0.61^{\star \star} \\
(0.25)\end{array}$ & $\begin{array}{c}-0.55^{\star \star} \\
(0.25)\end{array}$ & $\begin{array}{c}-0.92^{\star \star \star} \\
(0.34)\end{array}$ & $\begin{array}{c}-0.55^{\star \star} \\
(0.25)\end{array}$ & $\begin{array}{l}-0.28 \\
(0.31)\end{array}$ & $\begin{array}{l}-0.58 \\
(0.46)\end{array}$ & $\begin{array}{l}-0.82^{*} \\
(0.48)\end{array}$ & $\begin{array}{l}-0.72 \\
(0.50)\end{array}$ & $\begin{array}{l}-0.70 \\
(0.44)\end{array}$ & $\begin{array}{l}-0.50 \\
(0.45)\end{array}$ \\
\hline $\begin{array}{l}\text { Effective Average } \\
\text { Corporate tax rate }_{j t}\end{array}$ & $\begin{array}{l}-0.78^{* *} \\
(0.31)\end{array}$ & $\begin{array}{c}-0.98^{* * *} \\
(0.32)\end{array}$ & $\begin{array}{l}-0.74^{* *} \\
(0.37)\end{array}$ & $\begin{array}{c}-0.67^{* *} \\
(0.31)\end{array}$ & $\begin{array}{l}-0.64 \\
(0.39)\end{array}$ & $\begin{array}{l}-0.20 \\
(0.55)\end{array}$ & $\begin{array}{l}-0.0099 \\
(0.58)\end{array}$ & $\begin{array}{l}0.31 \\
(0.65)\end{array}$ & $\begin{array}{l}-0.13 \\
(0.56)\end{array}$ & $\begin{array}{l}-0.19 \\
(0.52)\end{array}$ \\
\hline $\mathrm{CRP}_{\mathrm{it}}$ & $\begin{array}{l}-0.13^{\star *} \\
(0.052)\end{array}$ & & $\begin{array}{l}-0.12^{\star \star} \\
(0.049)\end{array}$ & $\begin{array}{l}-0.14^{\star * *} \\
(0.048)\end{array}$ & $\begin{array}{l}-0.16^{\star * *} \\
(0.053)\end{array}$ & $\begin{array}{l}0.0078 \\
(0.070)\end{array}$ & & $\begin{array}{c}0.022 \\
(0.069)\end{array}$ & $\begin{array}{l}-0.014 \\
(0.068)\end{array}$ & $\begin{array}{l}-0.028 \\
(0.061)\end{array}$ \\
\hline $\mathrm{EEA}_{\mathrm{it}}$ & $\begin{array}{l}-0.21 \\
(0.23)\end{array}$ & $\begin{array}{l}0.095 \\
(0.25)\end{array}$ & $\begin{array}{l}-0.076 \\
(0.24)\end{array}$ & $\begin{array}{l}-0.62^{* *} \\
(0.28)\end{array}$ & $\begin{array}{c}0.36 \\
(0.23)\end{array}$ & $\begin{array}{l}0.98^{* \star *} \\
(0.31)\end{array}$ & $\begin{array}{l}1.14^{* * *} \\
(0.31)\end{array}$ & $\begin{array}{l}1.14^{* * *} \\
(0.32)\end{array}$ & $\begin{array}{l}0.95^{\star \star \star} \\
(0.30)\end{array}$ & $\begin{array}{l}1.07^{* \star \star} \\
(0.27)\end{array}$ \\
\hline $\mathrm{EEA}_{\mathrm{jt}}$ & $\begin{array}{l}0.64^{*} \\
(0.37)\end{array}$ & $\begin{array}{c}0.60 \\
(0.39)\end{array}$ & $\begin{array}{l}1.04^{* *} \\
(0.53)\end{array}$ & $\begin{array}{c}0.32 \\
(0.36)\end{array}$ & $\begin{array}{c}0.31 \\
(0.33)\end{array}$ & $\begin{array}{l}-0.084 \\
(0.37)\end{array}$ & $\begin{array}{l}-0.051 \\
(0.37)\end{array}$ & $\begin{array}{c}0.12 \\
(0.38)\end{array}$ & $\begin{array}{l}-0.10 \\
(0.39)\end{array}$ & $\begin{array}{c}0.31 \\
(0.35)\end{array}$ \\
\hline $\mathrm{EEA}_{\mathrm{ijt}}$ & $\begin{array}{c}0.34 \\
(0.26)\end{array}$ & $\begin{array}{c}0.32 \\
(0.26)\end{array}$ & $\begin{array}{c}0.33 \\
(0.26)\end{array}$ & $\begin{array}{l}0.57^{\star *} \\
(0.26)\end{array}$ & $\begin{array}{c}0.19 \\
(0.22)\end{array}$ & $\begin{array}{c}0.11 \\
(0.20)\end{array}$ & $\begin{array}{l}0.090 \\
(0.20)\end{array}$ & $\begin{array}{l}0.084 \\
(0.21)\end{array}$ & $\begin{array}{c}0.11 \\
(0.21)\end{array}$ & $\begin{array}{c}0.10 \\
(0.19)\end{array}$ \\
\hline $\mathrm{EA}_{\mathrm{ijt}}$ & $\begin{array}{c}0.13 \\
(0.15)\end{array}$ & $\begin{array}{c}0.13 \\
(0.15)\end{array}$ & $\begin{array}{c}0.13 \\
(0.15)\end{array}$ & $\begin{array}{c}0.14 \\
(0.12)\end{array}$ & $\begin{array}{l}-0.061 \\
(0.15)\end{array}$ & $\begin{array}{c}0.22 \\
(0.17)\end{array}$ & $\begin{array}{c}0.25 \\
(0.17)\end{array}$ & $\begin{array}{l}0.21 \\
(0.18)\end{array}$ & $\begin{array}{c}0.27 \\
(0.17)\end{array}$ & $\begin{array}{l}0.064 \\
(0.15)\end{array}$ \\
\hline NAFTA $_{i j}$ & $\begin{array}{c}-1.22^{* \star *} \\
(0.28)\end{array}$ & $\begin{array}{c}-1.23^{\star \star \star} \\
(0.29)\end{array}$ & $\begin{array}{l}-1.22^{\star \star \star} \\
(0.29)\end{array}$ & $\begin{array}{c}-0.84^{* * *} \\
(0.27)\end{array}$ & $\begin{array}{c}-1.01^{* * *} \\
(0.24)\end{array}$ & $\begin{array}{l}-1.13^{*} \\
(0.59)\end{array}$ & $\begin{array}{l}-1.14^{*} \\
(0.58)\end{array}$ & $\begin{array}{l}-1.16^{* *} \\
(0.58)\end{array}$ & $\begin{array}{l}-1.38^{* *} \\
(0.60)\end{array}$ & $\begin{array}{l}-1.00^{*} \\
(0.56)\end{array}$ \\
\hline $\mathrm{EPL}_{\text {it }}$ & $\begin{array}{l}-0.14 \\
(0.17)\end{array}$ & $\begin{array}{l}-0.10 \\
(0.18)\end{array}$ & $\begin{array}{l}-0.25 \\
(0.19)\end{array}$ & $\begin{array}{l}-0.030 \\
(0.17)\end{array}$ & $\begin{array}{l}-0.21 \\
(0.17)\end{array}$ & $\begin{array}{l}-0.014 \\
(0.19)\end{array}$ & $\begin{array}{l}-0.21 \\
(0.20)\end{array}$ & $\begin{array}{l}-0.21 \\
(0.21)\end{array}$ & $\begin{array}{l}-0.054 \\
(0.20)\end{array}$ & $\begin{array}{c}-0.00035 \\
(0.18)\end{array}$ \\
\hline$E L_{j t}$ & $\begin{array}{l}-0.49^{*} \\
(0.27)\end{array}$ & $\begin{array}{l}-0.51^{*} \\
(0.27)\end{array}$ & $\begin{array}{l}-0.40 \\
(0.26)\end{array}$ & $\begin{array}{l}-0.47^{*} \\
(0.27)\end{array}$ & $\begin{array}{l}-0.38 \\
(0.35)\end{array}$ & $\begin{array}{l}-0.12 \\
(0.25)\end{array}$ & $\begin{array}{l}-0.083 \\
(0.24)\end{array}$ & $\begin{array}{c}0.11 \\
(0.26)\end{array}$ & $\begin{array}{l}-0.054 \\
(0.26)\end{array}$ & $\begin{array}{l}-0.28 \\
(0.24)\end{array}$ \\
\hline Tariff $_{\text {it }}$ & $\begin{array}{l}-0.13 \\
(0.12)\end{array}$ & & & $\begin{array}{l}-0.12 \\
(0.11)\end{array}$ & $\begin{array}{l}0.065 \\
(0.10)\end{array}$ & $\begin{array}{l}0.39^{* \star \star} \\
(0.14)\end{array}$ & & & $\begin{array}{l}0.38^{\star \star \star} \\
(0.14)\end{array}$ & $\begin{array}{l}0.40^{* \star \star} \\
(0.12)\end{array}$ \\
\hline $\operatorname{Tariff}_{j t}$ & $\begin{array}{l}0.17^{*} \\
(0.10)\end{array}$ & & & $\begin{array}{l}0.20^{* *} \\
(0.099)\end{array}$ & $\begin{array}{l}0.0099 \\
(0.14)\end{array}$ & $\begin{array}{l}-0.13 \\
(0.13)\end{array}$ & & & $\begin{array}{l}-0.14 \\
(0.13)\end{array}$ & $\begin{array}{l}-0.23^{*} \\
(0.12)\end{array}$ \\
\hline FDI rest. index ${ }_{i t}$ & $\begin{array}{l}-0.044 \\
(0.14) \\
\end{array}$ & & & $\begin{array}{l}-0.17 \\
(0.17) \\
\end{array}$ & $\begin{array}{l}-0.053 \\
(0.13) \\
\end{array}$ & $\begin{array}{c}0.13 \\
(0.14) \\
\end{array}$ & & & $\begin{array}{l}0.035 \\
(0.14) \\
\end{array}$ & $\begin{array}{l}0.077 \\
(0.13) \\
\end{array}$ \\
\hline$N$ & 2024 & 2024 & 2024 & 1958 & 2462 & 2024 & 2024 & 2024 & 1958 & 2462 \\
\hline Sample & $\begin{array}{c}0.899 \\
\text { OECD31 }\end{array}$ & $\begin{array}{c}0.898 \\
\text { OECD31 }\end{array}$ & $\begin{array}{c}0.897 \\
\text { OECD31 }\end{array}$ & $\begin{array}{c}0.917 \\
\text { OECD31 }\end{array}$ & $\begin{array}{c}0.855 \\
\text { OECD34 }\end{array}$ & $\begin{array}{c}0.817 \\
\text { OECD31 }\end{array}$ & $\begin{array}{c}0.817 \\
\text { OECD31 }\end{array}$ & $\begin{array}{c}0.818 \\
\text { OECD31 }\end{array}$ & $\begin{array}{c}0.819 \\
\text { OECD31 }\end{array}$ & $\begin{array}{c}0.821 \\
\text { OECD34 }\end{array}$ \\
\hline $\begin{array}{l}\text { Country and year } \\
\text { fixed effects }\end{array}$ & YES & YES & YES & YES & YES & YES & YES & YES & YES & YES \\
\hline $\begin{array}{l}\text { Controls for PMR } \\
\text { levels in seven } \\
\text { subdomains }\end{array}$ & NO & NO & YES & NO & NO & NO & NO & YES & NO & NO \\
\hline $\begin{array}{l}\text { Controls for size and } \\
\text { factor dissimilarities }\end{array}$ & NO & NO & NO & YES & NO & NO & NO & NO & YES & NO \\
\hline
\end{tabular}

Note: Asterisks $\left({ }^{*},{ }^{* *},{ }^{* *}\right)$ indicate the significance level $(10 \%, 5 \%, 1 \%)$ of the coefficients. i denotes the inward country and $j$ the outward country. Standard errors adjusted for country-pair clusters are in parentheses. Controls for nominal GDP in US Dollars, geographical distance, contiguity, common official language, common type of legal system and nominal dollar exchange rate are also included. $\mathrm{CRP}_{\text {it }}=\mathrm{Complexity}_{\mathrm{s}}$ of regulatory

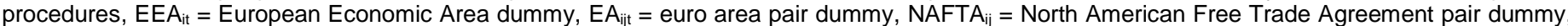
$\mathrm{PMR}_{\mathrm{it}}=$ Strictness of employment protection for regular contracts, Tariff ${ }_{\mathrm{it}}=\mathrm{PMR}$ indicator of trade tariffs, $\mathrm{PMR}_{\mathrm{it}}=$ Product market regulation overall indicator, $\mathrm{PMR}_{\mathrm{ijt}}^{\mathrm{h}}=$ Product market regulation indicator heterogeneity. Poisson = Poisson pseudo-maximum likelihood. OECD31 refers to all OECD countries except Hungary, Luxembourg and the Netherlands that have the highest share of Special Purpose entities, OEC34 refers to all OECD countries. 


\section{$\mathrm{ECO} / \mathrm{WKP}(2015) 86$}

Table A1.4. FDI determinants: Excluding countries with large FDI flows

\begin{tabular}{|c|c|c|c|c|c|c|c|c|}
\hline Estimation method & $\begin{array}{c}(1) \\
\text { Poisson }\end{array}$ & $\begin{array}{c}(2) \\
\text { Poisson }\end{array}$ & $\begin{array}{c}\text { (3) } \\
\text { Poisson }\end{array}$ & $\begin{array}{c}(4) \\
\text { Poisson }\end{array}$ & $\begin{array}{c}(5) \\
\text { Linear }\end{array}$ & $\begin{array}{c}(6) \\
\text { Linear }\end{array}$ & $\begin{array}{c}(7) \\
\text { Linear }\end{array}$ & $\begin{array}{c}(8) \\
\text { Linear }\end{array}$ \\
\hline $\ln \left(\mathrm{Y}_{\mathrm{it}}\right)$ & $\begin{array}{c}1.09^{\star \star \star} \\
(0.29)\end{array}$ & $\begin{array}{c}1.25^{\star \star \star} \\
(0.25)\end{array}$ & $\begin{array}{c}1.33^{\star \star \star} \\
(0.32)\end{array}$ & $\begin{array}{c}0.35 \\
(0.30)\end{array}$ & $\begin{array}{l}0.69^{*} \\
(0.37)\end{array}$ & $\begin{array}{l}0.74^{\star *} \\
(0.32)\end{array}$ & $\begin{array}{l}1.00^{\star \star} \\
(0.41)\end{array}$ & $\begin{array}{c}0.61 \\
(0.37)\end{array}$ \\
\hline $\ln \left(Y_{j t}\right)$ & $\begin{array}{c}1.38^{\star \star \star} \\
(0.32)\end{array}$ & $\begin{array}{l}1.19^{\star * *} \\
(0.29)\end{array}$ & $\begin{array}{l}1.20^{\star * *} \\
(0.41)\end{array}$ & $\begin{array}{l}0.61^{\star *} \\
(0.25)\end{array}$ & $\begin{array}{l}1.33^{\star \star \star} \\
(0.33)\end{array}$ & $\begin{array}{l}1.49^{\star * \star} \\
(0.33)\end{array}$ & $\begin{array}{c}1.40^{\star * *} \\
(0.42)\end{array}$ & $\begin{array}{c}1.20^{\star * *} \\
(0.33)\end{array}$ \\
\hline $\ln \left(\right.$ dist $\left._{i j}\right)$ & $\begin{array}{c}-1.04^{* * *} \\
(0.11)\end{array}$ & $\begin{array}{c}-1.03^{* * *} \\
(0.11)\end{array}$ & $\begin{array}{c}-1.03^{\star * *} \\
(0.11)\end{array}$ & $\begin{array}{c}-0.90^{* * *} \\
(0.079)\end{array}$ & $\begin{array}{c}-1.30^{* * *} \\
(0.12)\end{array}$ & $\begin{array}{c}-1.30^{* * *} \\
(0.12)\end{array}$ & $\begin{array}{c}-1.30^{* * *} \\
(0.12)\end{array}$ & $\begin{array}{c}-1.30^{* * *} \\
(0.12)\end{array}$ \\
\hline Contiguity $_{\mathrm{ij}}$ & $\begin{array}{c}0.20 \\
(0.17)\end{array}$ & $\begin{array}{c}0.20 \\
(0.17)\end{array}$ & $\begin{array}{c}0.21 \\
(0.17)\end{array}$ & $\begin{array}{l}0.22^{*} \\
(0.13)\end{array}$ & $\begin{array}{r}-0.037 \\
(0.25)\end{array}$ & $\begin{array}{c}-0.035 \\
(0.24)\end{array}$ & $\begin{array}{r}-0.037 \\
(0.25)\end{array}$ & $\begin{array}{c}-0.050 \\
(0.25)\end{array}$ \\
\hline Same legal system $_{i j}$ & $\begin{array}{c}0.13 \\
(0.13)\end{array}$ & $\begin{array}{c}0.14 \\
(0.13)\end{array}$ & $\begin{array}{c}0.13 \\
(0.13)\end{array}$ & $\begin{array}{l}0.094 \\
(0.11)\end{array}$ & $\begin{array}{c}0.47^{\star \star *} \\
(0.14)\end{array}$ & $\begin{array}{c}0.47^{\star * *} \\
(0.14)\end{array}$ & $\begin{array}{c}0.46^{* * *} \\
(0.14)\end{array}$ & $\begin{array}{c}0.47^{\star * *} \\
(0.15)\end{array}$ \\
\hline Language $_{i j}$ & $\begin{array}{c}0.53^{\star * *} \\
(0.19)\end{array}$ & $\begin{array}{c}0.53^{* * *} \\
(0.19)\end{array}$ & $\begin{array}{c}0.54^{* * *} \\
(0.19)\end{array}$ & $\begin{array}{c}0.57^{* * *} \\
(0.15)\end{array}$ & $\begin{array}{c}1.40^{\star * *} \\
(0.28)\end{array}$ & $\begin{array}{c}1.41^{* * *} \\
(0.28)\end{array}$ & $\begin{array}{c}1.40^{* * *} \\
(0.28)\end{array}$ & $\begin{array}{c}1.43^{* * *} \\
(0.29)\end{array}$ \\
\hline $\ln \left(\right.$ Remoteness $\left._{\mathrm{it}}\right)$ & $\begin{array}{c}0.63 \\
(1.10)\end{array}$ & $\begin{array}{c}0.81 \\
(1.13)\end{array}$ & $\begin{array}{c}0.46 \\
(1.18)\end{array}$ & $\begin{array}{c}-0.49 \\
(1.06)\end{array}$ & $\begin{array}{c}2.31 \\
(2.11)\end{array}$ & $\begin{array}{c}1.62 \\
(2.15)\end{array}$ & $\begin{array}{c}2.15 \\
(2.23)\end{array}$ & $\begin{array}{c}1.80 \\
(2.15)\end{array}$ \\
\hline $\ln \left(\right.$ Remoteness $\left._{\mathrm{jt}}\right)$ & $\begin{array}{l}-2.21^{*} \\
(1.32)\end{array}$ & $\begin{array}{c}-2.82^{\star *} \\
(1.37)\end{array}$ & $\begin{array}{l}-3.01^{*} \\
(1.60)\end{array}$ & $\begin{array}{c}-3.19^{* \star *} \\
(1.13)\end{array}$ & $\begin{array}{l}-1.70 \\
(2.11)\end{array}$ & $\begin{array}{l}-1.02 \\
(2.05)\end{array}$ & $\begin{array}{l}-0.13 \\
(2.25)\end{array}$ & $\begin{array}{l}-1.98 \\
(2.13)\end{array}$ \\
\hline $\ln \left(\right.$ Exch. rate $\left._{\text {it }}\right)$ & $\begin{array}{c}-0.55 \\
(0.37)\end{array}$ & $\begin{array}{c}-0.84^{* *} \\
(0.37)\end{array}$ & $\begin{array}{c}-0.73^{\star *} \\
(0.37)\end{array}$ & $\begin{array}{c}-0.17 \\
(0.37)\end{array}$ & $\begin{array}{l}-0.96^{*} \\
(0.52)\end{array}$ & $\begin{array}{l}-0.92^{*} \\
(0.52)\end{array}$ & $\begin{array}{l}-0.94^{*} \\
(0.53)\end{array}$ & $\begin{array}{l}-0.67 \\
(0.54)\end{array}$ \\
\hline $\ln \left(\right.$ Exch. rate $\left._{\mathrm{jt}}\right)$ & $\begin{array}{c}-1.05^{\star *} \\
(0.52)\end{array}$ & $\begin{array}{l}-0.80 \\
(0.53)\end{array}$ & $\begin{array}{l}-1.07^{*} \\
(0.57)\end{array}$ & $\begin{array}{l}-0.63 \\
(0.44)\end{array}$ & $\begin{array}{c}0.10 \\
(0.35)\end{array}$ & $\begin{array}{l}-0.024 \\
(0.34)\end{array}$ & $\begin{array}{c}0.12 \\
(0.40)\end{array}$ & $\begin{array}{l}0.058 \\
(0.36)\end{array}$ \\
\hline $\mathrm{PMR}_{i t}$ & & $\begin{array}{l}-0.21 \\
(0.17)\end{array}$ & & & & $\begin{array}{l}0.50^{*} \\
(0.28)\end{array}$ & & \\
\hline $\mathrm{PMR}_{j t}$ & & $\begin{array}{c}-0.0052 \\
(0.19)\end{array}$ & & & & $\begin{array}{c}-0.40 \\
(0.27)\end{array}$ & & \\
\hline $\mathrm{PMR}_{i j t}^{h}$ & $\begin{array}{c}-1.86^{\star * *} \\
(0.70)\end{array}$ & $\begin{array}{c}-1.79^{* *} \\
(0.71)\end{array}$ & $\begin{array}{c}-1.73^{\star *} \\
(0.76)\end{array}$ & $\begin{array}{l}-1.04^{*} \\
(0.57)\end{array}$ & $\begin{array}{c}-2.28^{* *} \\
(0.88)\end{array}$ & $\begin{array}{c}-2.23^{\star *} \\
(0.89)\end{array}$ & $\begin{array}{c}-2.28^{\star *} \\
(0.92)\end{array}$ & $\begin{array}{c}-2.09^{* *} \\
(0.89)\end{array}$ \\
\hline $\mathrm{CRP}_{\text {it }}$ & $\begin{array}{l}-0.11^{\star *} \\
(0.045)\end{array}$ & & $\begin{array}{l}-0.12^{* * *} \\
(0.043)\end{array}$ & $\begin{array}{c}-0.095^{\star *} \\
(0.047)\end{array}$ & $\begin{array}{c}0.081 \\
(0.078)\end{array}$ & & $\begin{array}{c}0.075 \\
(0.078)\end{array}$ & $\begin{array}{c}0.053 \\
(0.077)\end{array}$ \\
\hline $\mathrm{EEA}_{\mathrm{it}}$ & $\begin{array}{c}0.79^{* * *} \\
(0.30)\end{array}$ & $\begin{array}{c}0.77^{\star * *} \\
(0.29)\end{array}$ & $\begin{array}{l}0.66^{* *} \\
(0.31)\end{array}$ & $\begin{array}{l}0.51^{*} \\
(0.26)\end{array}$ & $\begin{array}{c}1.27^{* * *} \\
(0.33)\end{array}$ & $\begin{array}{c}0.93^{* * *} \\
(0.30)\end{array}$ & $\begin{array}{c}0.92^{* * *} \\
(0.32)\end{array}$ & $\begin{array}{c}1.15^{\star \star \star} \\
(0.32)\end{array}$ \\
\hline$E E A_{j t}$ & $\begin{array}{l}1.51^{\star \star *} \\
(0.40)\end{array}$ & $\begin{array}{l}1.36^{\star \star *} \\
(0.41)\end{array}$ & $\begin{array}{c}2.62^{\star * *} \\
(0.72)\end{array}$ & $\begin{array}{l}1.09^{* * *} \\
(0.34)\end{array}$ & $\begin{array}{l}0.017 \\
(0.39)\end{array}$ & $\begin{array}{c}-0.039 \\
(0.38)\end{array}$ & $\begin{array}{c}0.12 \\
(0.39)\end{array}$ & $\begin{array}{c}-0.018 \\
(0.39)\end{array}$ \\
\hline $\mathrm{EEA}_{\mathrm{ijt}}$ & $\begin{array}{c}-0.75^{\star *} \\
(0.30)\end{array}$ & $\begin{array}{c}-0.75^{\star *} \\
(0.30)\end{array}$ & $\begin{array}{c}-0.70^{\star *} \\
(0.30)\end{array}$ & $\begin{array}{c}-0.49^{\star *} \\
(0.22)\end{array}$ & $\begin{array}{l}-0.13 \\
(0.23)\end{array}$ & $\begin{array}{l}-0.15 \\
(0.23)\end{array}$ & $\begin{array}{l}-0.14 \\
(0.23)\end{array}$ & $\begin{array}{c}-0.094 \\
(0.23)\end{array}$ \\
\hline$E A_{i j t}$ & $\begin{array}{l}0.064 \\
(0.15)\end{array}$ & $\begin{array}{l}0.061 \\
(0.15)\end{array}$ & $\begin{array}{l}0.021 \\
(0.16)\end{array}$ & $\begin{array}{c}0.16 \\
(0.12)\end{array}$ & $\begin{array}{c}0.21 \\
(0.19)\end{array}$ & $\begin{array}{c}0.21 \\
(0.19)\end{array}$ & $\begin{array}{c}0.18 \\
(0.20)\end{array}$ & $\begin{array}{c}0.25 \\
(0.19)\end{array}$ \\
\hline $\mathrm{NAFTA}_{i j}$ & $\begin{array}{c}-1.71^{* * *} \\
(0.32)\end{array}$ & $\begin{array}{c}-1.69^{* * *} \\
(0.33)\end{array}$ & $\begin{array}{c}-1.71^{* * *} \\
(0.33)\end{array}$ & $\begin{array}{c}-1.14^{* * *} \\
(0.27)\end{array}$ & $\begin{array}{c}-1.43^{* *} \\
(0.70)\end{array}$ & $\begin{array}{c}-1.43^{\star *} \\
(0.70)\end{array}$ & $\begin{array}{c}-1.45^{\star *} \\
(0.69)\end{array}$ & $\begin{array}{c}-1.59^{* *} \\
(0.71)\end{array}$ \\
\hline $\mathrm{EPL}_{\text {it }}$ & $\begin{array}{l}-0.19 \\
(0.19)\end{array}$ & $\begin{array}{l}-0.17 \\
(0.21)\end{array}$ & $\begin{array}{c}-0.26 \\
(0.22)\end{array}$ & $\begin{array}{r}-0.068 \\
(0.16)\end{array}$ & $\begin{array}{r}-0.027 \\
(0.20)\end{array}$ & $\begin{array}{c}-0.23 \\
(0.21)\end{array}$ & $\begin{array}{c}-0.29 \\
(0.23)\end{array}$ & $\begin{array}{c}-0.0028 \\
(0.20)\end{array}$ \\
\hline$E P L_{j t}$ & $\begin{array}{l}-0.49^{*} \\
(0.30)\end{array}$ & $\begin{array}{l}-0.51^{*} \\
(0.30)\end{array}$ & $\begin{array}{l}-0.30 \\
(0.27)\end{array}$ & $\begin{array}{l}-0.39 \\
(0.26)\end{array}$ & $\begin{array}{c}-0.29 \\
(0.25)\end{array}$ & $\begin{array}{l}-0.23 \\
(0.24)\end{array}$ & $\begin{array}{l}-0.11 \\
(0.25)\end{array}$ & $\begin{array}{c}-0.23 \\
(0.26)\end{array}$ \\
\hline Tariff $_{\text {it }}$ & $\begin{array}{l}0.067 \\
(0.12)\end{array}$ & & & $\begin{array}{l}0.045 \\
(0.11)\end{array}$ & $\begin{array}{c}0.53^{* * *} \\
(0.16)\end{array}$ & & & $\begin{array}{c}0.48^{* * *} \\
(0.16)\end{array}$ \\
\hline Tariff $_{j t}$ & $\begin{array}{l}0.19^{*} \\
(0.11)\end{array}$ & & & $\begin{array}{c}0.20^{*} \\
(0.11)\end{array}$ & $\begin{array}{l}-0.12 \\
(0.13)\end{array}$ & & & $\begin{array}{l}-0.12 \\
(0.13)\end{array}$ \\
\hline FDI rest. index $i t$ & $\begin{array}{c}-0.35^{\star *} \\
(0.14) \\
\end{array}$ & & & $\begin{array}{c}-0.37^{\star *} \\
(0.17) \\
\end{array}$ & $\begin{array}{l}-0.074 \\
(0.14) \\
\end{array}$ & & & $\begin{array}{l}-0.17 \\
(0.14) \\
\end{array}$ \\
\hline$N$ & 1688 & 1688 & 1688 & 1655 & 1688 & 1688 & 1688 & 1655 \\
\hline Sample & $\begin{array}{c}0.920 \\
\text { OECD29 }\end{array}$ & $\begin{array}{c}0.917 \\
\text { OECD29 }\end{array}$ & $\begin{array}{c}0.919 \\
\text { OECD29 }\end{array}$ & $\begin{array}{c}0.948 \\
\text { OECD29 }\end{array}$ & $\begin{array}{c}0.822 \\
\text { OECD29 }\end{array}$ & $\begin{array}{c}0.821 \\
\text { OECD29 }\end{array}$ & $\begin{array}{c}0.822 \\
\text { OECD29 }\end{array}$ & $\begin{array}{c}0.825 \\
\text { OECD29 }\end{array}$ \\
\hline $\begin{array}{l}\text { Country and year } \\
\text { fixed effects }\end{array}$ & YES & YES & YES & YES & YES & YES & YES & YES \\
\hline $\begin{array}{l}\text { Controls for PMR levels } \\
\text { in seven subdomains }\end{array}$ & NO & NO & YES & NO & NO & $\mathrm{NO}$ & YES & NO \\
\hline $\begin{array}{l}\text { Controls for size and } \\
\text { factor dissimilarities }\end{array}$ & NO & NO & NO & YES & NO & $\mathrm{NO}$ & $\mathrm{NO}$ & YES \\
\hline
\end{tabular}

Note: Asterisks $\left({ }^{*},{ }^{* *},{ }^{* * *}\right)$ indicate the significance level $(10 \%, 5 \%, 1 \%)$ of the coefficients. i denotes the inward country and $\mathrm{j}$ the outward country.

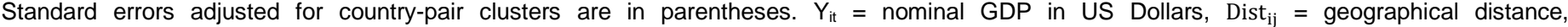

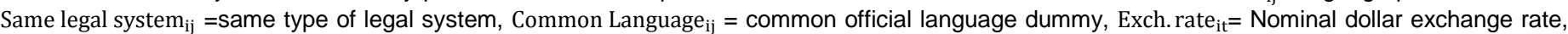
$\mathrm{CRP}_{i t}=$ Complexity of regulatory procedures, $\mathrm{EEA}_{\mathrm{it}}=$ European Economic Area dummy, $\mathrm{EA}_{\mathrm{ijt}}=$ euro area pair dummy, NAFTA $\mathrm{A}_{\mathrm{ij}}=\mathrm{North}_{\mathrm{American}} \mathrm{Free}$ Trade Agreement pair dummy, $\mathrm{PMR}_{\mathrm{it}}=$ Strictness of employment protection for regular contracts, Tariff ${ }_{\mathrm{it}}=\mathrm{PMR}_{\mathrm{indicator}}$ of trade tariffs, $\mathrm{PMR} \mathrm{P}_{\mathrm{it}}=$ Product market regulation overall indicator, $\mathrm{PMR}_{\mathrm{ijt}}^{\mathrm{h}}=$ Product market regulation indicator heterogeneity. $\mathrm{Poisson}=\mathrm{Poisson}$ pseudo-maximum likelihood. OECD29 refers to all OECD countries except Belgium, Ireland, Luxembourg, the Netherlands and Switzerland where FDI flows relative to GDP are the highest. 
Table A1.5. The role of heterogeneity by regulation domain

\begin{tabular}{|c|c|c|c|c|c|c|c|c|c|c|}
\hline Estimation method & $\begin{array}{c}(1) \\
\text { Poisson } \\
\end{array}$ & $\begin{array}{c}(2) \\
\text { Poisson } \\
\end{array}$ & $\begin{array}{c}(3) \\
\text { Poisson } \\
\end{array}$ & $\begin{array}{c}(4) \\
\text { Poisson } \\
\end{array}$ & $\begin{array}{c}(5) \\
\text { Poisson } \\
\end{array}$ & $\begin{array}{c}(6) \\
\text { Linear }\end{array}$ & $\begin{array}{c}(7) \\
\text { Linear }\end{array}$ & $\begin{array}{c}(8) \\
\text { Linear }\end{array}$ & $\begin{array}{c}(9) \\
\text { Linear }\end{array}$ & $\begin{array}{l}(10) \\
\text { Linear }\end{array}$ \\
\hline Public ownership $\mathrm{i}_{\mathrm{ijt}}^{\mathrm{h}}$ & $\begin{array}{l}0.066 \\
(0.16)\end{array}$ & $\begin{array}{l}0.049 \\
(0.15)\end{array}$ & $\begin{array}{c}0.11 \\
(0.16)\end{array}$ & $\begin{array}{c}0.41^{\star * *} \\
(0.16)\end{array}$ & $\begin{array}{l}-0.0096 \\
(0.16)\end{array}$ & $\begin{array}{c}-0.69^{* * *} \\
(0.26)\end{array}$ & $\begin{array}{l}-0.65^{\star *} \\
(0.26)\end{array}$ & $\begin{array}{l}-0.63^{* *} \\
(0.26)\end{array}$ & $\begin{array}{l}-0.54^{\star *} \\
(0.27)\end{array}$ & $\begin{array}{l}-0.48^{* *} \\
(0.24)\end{array}$ \\
\hline Involv. in business ${ }_{\mathrm{ijt}}^{\mathrm{h}}$ & $\begin{array}{l}-0.41 \\
(0.42)\end{array}$ & $\begin{array}{l}-0.44 \\
(0.41)\end{array}$ & $\begin{array}{l}-0.31 \\
(0.42)\end{array}$ & $\begin{array}{l}-0.56 \\
(0.39)\end{array}$ & $\begin{array}{l}-0.64^{*} \\
(0.38)\end{array}$ & $\begin{array}{l}-0.32 \\
(0.57)\end{array}$ & $\begin{array}{l}-0.35 \\
(0.56)\end{array}$ & $\begin{array}{l}-0.36 \\
(0.59)\end{array}$ & $\begin{array}{l}-0.12 \\
(0.60)\end{array}$ & $\begin{array}{l}-0.37 \\
(0.50)\end{array}$ \\
\hline Complexity $_{\mathrm{ijt}}^{\mathrm{h}}$ & $\begin{array}{c}0.42 \\
(0.34)\end{array}$ & $\begin{array}{c}0.37 \\
(0.34)\end{array}$ & $\begin{array}{c}0.35 \\
(0.34)\end{array}$ & $\begin{array}{c}0.42 \\
(0.32)\end{array}$ & $\begin{array}{l}0.018 \\
(0.35)\end{array}$ & $\begin{array}{l}0.15 \\
(0.41)\end{array}$ & $\begin{array}{l}0.043 \\
(0.41)\end{array}$ & $\begin{array}{l}0.081 \\
(0.42)\end{array}$ & $\begin{array}{c}0.19 \\
(0.41)\end{array}$ & $\begin{array}{l}0.041 \\
(0.39)\end{array}$ \\
\hline Adm. burden ${ }_{\mathrm{ijt}}^{\mathrm{h}}$ & $\begin{array}{c}0.21 \\
(0.33)\end{array}$ & $\begin{array}{l}0.079 \\
(0.34)\end{array}$ & $\begin{array}{c}0.28 \\
(0.37)\end{array}$ & $\begin{array}{c}0.18 \\
(0.33)\end{array}$ & $\begin{array}{l}-0.44 \\
(0.31)\end{array}$ & $\begin{array}{l}-0.45 \\
(0.45)\end{array}$ & $\begin{array}{l}-0.45 \\
(0.45)\end{array}$ & $\begin{array}{l}-0.42 \\
(0.47)\end{array}$ & $\begin{array}{l}-0.88^{*} \\
(0.47)\end{array}$ & $\begin{array}{l}0.073 \\
(0.42)\end{array}$ \\
\hline Incumbents prot: ${ }_{\text {ijt }}$ & $\begin{array}{c}-2.55^{\star * *} \\
(0.53)\end{array}$ & $\begin{array}{c}-2.59^{* * *} \\
(0.53)\end{array}$ & $\begin{array}{c}-2.88^{* * *} \\
(0.57)\end{array}$ & $\begin{array}{c}-1.70^{* * *} \\
(0.46)\end{array}$ & $\begin{array}{c}-2.42^{* * *} \\
(0.44)\end{array}$ & $\begin{array}{c}-3.19^{* * *} \\
(0.74)\end{array}$ & $\begin{array}{c}-3.24^{* * *} \\
(0.74)\end{array}$ & $\begin{array}{c}-3.37^{\star * *} \\
(0.76)\end{array}$ & $\begin{array}{c}-2.62^{* * *} \\
(0.76)\end{array}$ & $\begin{array}{c}-3.06^{* * *} \\
(0.69)\end{array}$ \\
\hline $\mathrm{PMR}_{\mathrm{it}}$ & & $\begin{array}{l}-0.023 \\
(0.22)\end{array}$ & & & & & $\begin{array}{l}0.83^{\star *} \\
(0.37)\end{array}$ & & & \\
\hline $\mathrm{PMR}_{\mathrm{jt}}$ & & $\begin{array}{c}0.26 \\
(0.25)\end{array}$ & & & & & $\begin{array}{c}0.15 \\
(0.34)\end{array}$ & & & \\
\hline $\mathrm{CRP}_{\text {it }}$ & $\begin{array}{l}-0.20^{\star *} \\
(0.086)\end{array}$ & & $\begin{array}{l}-0.22^{\star \star} \\
(0.090)\end{array}$ & $\begin{array}{l}-0.23^{* * *} \\
(0.071) \\
\end{array}$ & $\begin{array}{l}-0.25^{\star * *} \\
(0.074) \\
\end{array}$ & $\begin{array}{c}0.16 \\
(0.11) \\
\end{array}$ & & $\begin{array}{c}0.17 \\
(0.12)\end{array}$ & $\begin{array}{c}0.12 \\
(0.11)\end{array}$ & $\begin{array}{c}0.12 \\
(0.093) \\
\end{array}$ \\
\hline $\mathrm{N}$ & 1679 & 1679 & 1679 & 1613 & 2037 & 1679 & 1679 & 1679 & 1613 & 2037 \\
\hline $\mathrm{R}^{2}$ & 0.898 & 0.897 & 0.901 & 0.919 & 0.845 & 0.817 & 0.817 & 0.818 & 0.819 & 0.818 \\
\hline Sample & OECD31 & OECD31 & OECD31 & OECD31 & OECD34 & OECD31 & OECD31 & OECD31 & OECD31 & OECD34 \\
\hline $\begin{array}{l}\text { Country and year } \\
\text { fixed effects }\end{array}$ & YES & YES & YES & YES & YES & YES & YES & YES & YES & YES \\
\hline $\begin{array}{l}\text { Controls for PMR } \\
\text { levels in } 7 \text { sub- } \\
\text { domains }\end{array}$ & NO & NO & YES & NO & NO & NO & NO & YES & NO & NO \\
\hline $\begin{array}{l}\text { Controls for size and } \\
\text { factor dissimilarities }\end{array}$ & NO & NO & NO & YES & NO & NO & NO & NO & YES & NO \\
\hline
\end{tabular}

Note: Asterisks $\left({ }^{*},{ }^{* *},{ }^{* * *}\right)$ indicate the significance level $(10 \%, 5 \%, 1 \%)$ of the coefficients. i denotes the inward country and $\mathrm{j}$ the outward country. Standard errors adjusted for country-pair clusters are in parentheses. The superscript "h" stands for "heterogeneity". $\mathrm{CRP}_{\mathrm{it}}=$ Complexity of regulatory procedures. Sector-specific heterogeneity measures are added on top of the baseline regressions shown in Table 1. Poisson = Poisson pseudo-maximum likelihood. OECD31 refers to all OECD countries except Hungary, Luxembourg and the Netherlands that have the highest share of Special Purpose entities, OEC34 refers to all OECD countries. 


\section{ECO/WKP(2015)86}

Table A1.6. The effect of stringency of product market regulation by sub-domain

\begin{tabular}{|c|c|c|c|c|c|c|}
\hline Estimation method & $\begin{array}{c}(1) \\
\text { Poisson }\end{array}$ & $\begin{array}{c}(2) \\
\text { Linear }\end{array}$ & $\begin{array}{c}(3) \\
\text { Poisson } \\
\end{array}$ & $\begin{array}{c}(4) \\
\text { Linear }\end{array}$ & $\begin{array}{c}(5) \\
\text { Poisson } \\
\end{array}$ & $\begin{array}{c}(6) \\
\text { Linear }\end{array}$ \\
\hline Public Ownership ${ }_{i t}$ & $\begin{array}{c}0.12 \\
(0.071)\end{array}$ & $\begin{array}{c}0.15 \\
(0.10)\end{array}$ & $\begin{array}{c}0.082 \\
(0.079)\end{array}$ & $\begin{array}{c}0.18 \\
(0.13)\end{array}$ & $\begin{array}{c}0.14^{*} \\
(0.078)\end{array}$ & $\begin{array}{c}0.13 \\
(0.10)\end{array}$ \\
\hline Public Ownership $_{j t}$ & $\begin{array}{c}-0.081 \\
(0.089)\end{array}$ & $\begin{array}{r}-0.083 \\
(0.11)\end{array}$ & $\begin{array}{l}-0.10 \\
(0.10)\end{array}$ & $\begin{array}{c}-0.014 \\
(0.14)\end{array}$ & $\begin{array}{c}-0.037 \\
(0.087)\end{array}$ & $\begin{array}{c}-0.083 \\
(0.12)\end{array}$ \\
\hline Involv. in business $i t$ & $\begin{array}{l}0.0059 \\
(0.083)\end{array}$ & $\begin{array}{c}0.14 \\
(0.13)\end{array}$ & $\begin{array}{l}-0.12 \\
(0.17)\end{array}$ & $\begin{array}{l}0.056 \\
(0.21)\end{array}$ & $\begin{array}{l}-0.073 \\
(0.089)\end{array}$ & $\begin{array}{c}0.10 \\
(0.13)\end{array}$ \\
\hline Involv. in business $_{j t}$ & $\begin{array}{l}-0.18^{*} \\
(0.10)\end{array}$ & $\begin{array}{l}-0.24^{\star} \\
(0.13)\end{array}$ & $\begin{array}{c}-0.28 \\
(0.18)\end{array}$ & $\begin{array}{r}-0.017 \\
(0.19)\end{array}$ & $\begin{array}{c}-0.24^{\star *} \\
(0.11)\end{array}$ & $\begin{array}{l}-0.25^{\star} \\
(0.13)\end{array}$ \\
\hline Complexity $_{i t}$ & $\begin{array}{c}-0.16^{\star * *} \\
(0.046)\end{array}$ & $\begin{array}{c}0.013 \\
(0.070)\end{array}$ & $\begin{array}{l}-0.22^{\star *} \\
(0.090)\end{array}$ & $\begin{array}{c}0.17 \\
(0.12)\end{array}$ & $\begin{array}{c}-0.17^{\star \star *} \\
(0.050)\end{array}$ & $\begin{array}{c}0.014 \\
(0.072)\end{array}$ \\
\hline Complexity $_{j t}$ & $\begin{array}{c}-0.043 \\
(0.055)\end{array}$ & $\begin{array}{l}0.0026 \\
(0.069)\end{array}$ & $\begin{array}{c}-0.092 \\
(0.078)\end{array}$ & $\begin{array}{c}-0.043 \\
(0.11)\end{array}$ & $\begin{array}{c}-0.065 \\
(0.057)\end{array}$ & $\begin{array}{r}-0.0068 \\
(0.070)\end{array}$ \\
\hline Adm. burden $i t$ & $\begin{array}{c}-0.086 \\
(0.085)\end{array}$ & $\begin{array}{c}0.15 \\
(0.13)\end{array}$ & $\begin{array}{l}0.090 \\
(0.14)\end{array}$ & $\begin{array}{l}0.065 \\
(0.23)\end{array}$ & $\begin{array}{c}-0.076 \\
(0.087)\end{array}$ & $\begin{array}{l}0.072 \\
(0.14)\end{array}$ \\
\hline Adm. burden $n_{j t}$ & $\begin{array}{c}-0.29^{\star *} \\
(0.14)\end{array}$ & $\begin{array}{c}0.10 \\
(0.15)\end{array}$ & $\begin{array}{l}-0.46^{*} \\
(0.25)\end{array}$ & $\begin{array}{l}-0.26 \\
(0.26)\end{array}$ & $\begin{array}{c}-0.32^{\star *} \\
(0.15)\end{array}$ & $\begin{array}{l}0.035 \\
(0.15)\end{array}$ \\
\hline Incumbents prot ${ }_{i t}$ & $\begin{array}{l}-0.089 \\
(0.100)\end{array}$ & $\begin{array}{l}-0.31^{*} \\
(0.16)\end{array}$ & $\begin{array}{c}0.27 \\
(0.25)\end{array}$ & $\begin{array}{c}0.23 \\
(0.29)\end{array}$ & $\begin{array}{l}-0.14 \\
(0.11)\end{array}$ & $\begin{array}{l}-0.32^{* *} \\
(0.16)\end{array}$ \\
\hline Incumbents prot $_{j t}$ & $\begin{array}{l}0.32^{* *} \\
(0.14)\end{array}$ & $\begin{array}{l}-0.12 \\
(0.18)\end{array}$ & $\begin{array}{c}0.98^{\star * *} \\
(0.25)\end{array}$ & $\begin{array}{c}0.50 \\
(0.34)\end{array}$ & $\begin{array}{l}0.31^{\star *} \\
(0.14)\end{array}$ & $\begin{array}{l}-0.18 \\
(0.18)\end{array}$ \\
\hline $\begin{array}{l}\text { Explicit barriers } \\
\text { to trade and invest. } \text { tit }\end{array}$ & $\begin{array}{l}-0.25 \\
(0.20)\end{array}$ & $\begin{array}{l}0.53^{\star *} \\
(0.26)\end{array}$ & $\begin{array}{l}-0.17 \\
(0.21)\end{array}$ & $\begin{array}{c}0.41 \\
(0.33)\end{array}$ & $\begin{array}{l}-0.18 \\
(0.20)\end{array}$ & $\begin{array}{l}0.45^{*} \\
(0.26)\end{array}$ \\
\hline $\begin{array}{l}\text { Explicit barriers } \\
\text { to trade and invest.jt }\end{array}$ & $\begin{array}{l}-0.090 \\
(0.21)\end{array}$ & $\begin{array}{l}-0.28 \\
(0.22)\end{array}$ & $\begin{array}{r}-0.063 \\
(0.21)\end{array}$ & $\begin{array}{l}-0.29 \\
(0.24)\end{array}$ & $\begin{array}{l}-0.13 \\
(0.23)\end{array}$ & $\begin{array}{l}-0.35 \\
(0.22)\end{array}$ \\
\hline $\begin{array}{l}\text { Other barriers } \\
\text { to trade and invest. } i t\end{array}$ & $\begin{array}{l}-0.019 \\
(0.071)\end{array}$ & $\begin{array}{c}0.033 \\
(0.091)\end{array}$ & $\begin{array}{c}0.24 \\
(0.15)\end{array}$ & $\begin{array}{r}-0.040 \\
(0.22)\end{array}$ & $\begin{array}{c}0.019 \\
(0.067)\end{array}$ & $\begin{array}{c}0.053 \\
(0.089)\end{array}$ \\
\hline $\begin{array}{l}\text { Other barriers } \\
\text { to trade and invest.jt }\end{array}$ & $\begin{array}{c}0.37^{\star \star \star} \\
(0.12)\end{array}$ & $\begin{array}{c}0.10 \\
(0.097)\end{array}$ & $\begin{array}{l}0.78^{\star * *} \\
(0.21)\end{array}$ & $\begin{array}{l}0.46^{*} \\
(0.26)\end{array}$ & $\begin{array}{c}0.48^{\star \star *} \\
(0.13)\end{array}$ & $\begin{array}{c}0.12 \\
(0.093)\end{array}$ \\
\hline $\begin{array}{l}\text { Columns showing the } \\
\text { other regression results }\end{array}$ & $\begin{array}{l}\text { Table 1; } \\
\text { column } 4\end{array}$ & $\begin{array}{l}\text { Table 1; } \\
\text { column } 9\end{array}$ & $\begin{array}{l}\text { Table A1.5; } \\
\text { column } 4\end{array}$ & $\begin{array}{l}\text { Table A1.5; } \\
\text { column } 9\end{array}$ & $\begin{array}{l}\text { Table } 3 ; \\
\text { column } 4\end{array}$ & $\begin{array}{l}\text { Table 3; } \\
\text { column } 9\end{array}$ \\
\hline
\end{tabular}

Note: Asterisks $\left({ }^{*},{ }^{* *},{ }^{* *}\right)$ indicate the significance level $(10 \%, 5 \%, 1 \%)$ of the coefficients. i denotes the inward country and $j$ the outward country. Standard errors adjusted for country-pair clusters are in parentheses. These variables are added on top of the baseline regressions shown in other tables. Poisson = Poisson pseudo-maximum likelihood. 
Table A1.7. FDI determinants: Detailed results with a 1990-2013 sample

\begin{tabular}{|c|c|c|c|c|c|c|c|c|c|c|}
\hline Estimation method & $\begin{array}{c}(1) \\
\text { Poisson }\end{array}$ & $\begin{array}{c}(2) \\
\text { Poisson }\end{array}$ & $\begin{array}{c}(3) \\
\text { Poisson }\end{array}$ & $\begin{array}{c}(4) \\
\text { Poisson }\end{array}$ & $\begin{array}{c}\text { (5) } \\
\text { Poisson }\end{array}$ & $\begin{array}{c}6) \\
\text { Linear }\end{array}$ & $\begin{array}{c}(7) \\
\text { Linear }\end{array}$ & $\begin{array}{c}(8) \\
\text { Linear }\end{array}$ & $\begin{array}{c}(9) \\
\text { Linear }\end{array}$ & $\begin{array}{c}(10) \\
\text { Linear }\end{array}$ \\
\hline $\ln \left(Y_{i t}\right)$ & $\begin{array}{c}0.81^{* \star *} \\
(0.15)\end{array}$ & $\begin{array}{c}0.33 \\
(0.21)\end{array}$ & $\begin{array}{c}0.94^{\star \star \star} \\
(0.18)\end{array}$ & $\begin{array}{c}0.63^{\star \star \star} \\
(0.16)\end{array}$ & $\begin{array}{c}0.55^{\star \star \star} \\
(0.17)\end{array}$ & $\begin{array}{c}0.66^{\star \star *} \\
(0.19)\end{array}$ & $\begin{array}{l}0.48^{\star *} \\
(0.22)\end{array}$ & $\begin{array}{c}0.61^{\star \star \star} \\
(0.19)\end{array}$ & $\begin{array}{c}0.59^{\star \star \star} \\
(0.20)\end{array}$ & $\begin{array}{c}0.63^{\star \star \star} \\
(0.17)\end{array}$ \\
\hline $\ln \left(Y_{\mathrm{jt}}\right)$ & $\begin{array}{c}0.76^{\star \star \star} \\
(0.18)\end{array}$ & $\begin{array}{c}0.76^{\star \star *} \\
(0.19)\end{array}$ & $\begin{array}{l}1.01^{* * *} \\
(0.25)\end{array}$ & $\begin{array}{c}0.88^{\star * *} \\
(0.21)\end{array}$ & $\begin{array}{c}0.80^{\star \star *} \\
(0.18)\end{array}$ & $\begin{array}{l}1.14^{\star \star *} \\
(0.21)\end{array}$ & $\begin{array}{c}0.95^{\star \star \star} \\
(0.23)\end{array}$ & $\begin{array}{c}1.14^{\star \star \star} \\
(0.22)\end{array}$ & $\begin{array}{c}1.04^{\star \star *} \\
(0.22)\end{array}$ & $\begin{array}{c}0.98^{\star \star \star} \\
(0.20)\end{array}$ \\
\hline $\ln \left(\right.$ dist $\left._{\mathrm{ij}}\right)$ & $\begin{array}{c}-0.60^{* * *} \\
(0.11)\end{array}$ & $\begin{array}{c}-0.57^{\star \star *} \\
(0.12)\end{array}$ & $\begin{array}{l}-0.74^{\star \star \star} \\
(0.087)\end{array}$ & $\begin{array}{l}-0.69^{\star \star *} \\
(0.076)\end{array}$ & $\begin{array}{c}-0.52^{\star * *} \\
(0.095)\end{array}$ & $\begin{array}{c}-1.10^{\star \star *} \\
(0.11)\end{array}$ & $\begin{array}{c}-1.02^{\star \star *} \\
(0.12)\end{array}$ & $\begin{array}{c}-1.11^{\star \star \star} \\
(0.11)\end{array}$ & $\begin{array}{c}-1.12^{\star \star \star} \\
(0.11)\end{array}$ & $\begin{array}{c}-1.00^{\star * *} \\
(0.10)\end{array}$ \\
\hline Contiguity $_{\mathrm{ij}}$ & $\begin{array}{l}0.082 \\
(0.15)\end{array}$ & $\begin{array}{c}0.15 \\
(0.15)\end{array}$ & $\begin{array}{c}0.0011 \\
(0.14)\end{array}$ & $\begin{array}{l}0.045 \\
(0.12)\end{array}$ & $\begin{array}{c}0.14 \\
(0.15)\end{array}$ & $\begin{array}{l}-0.12 \\
(0.22)\end{array}$ & $\begin{array}{c}-0.032 \\
(0.24)\end{array}$ & $\begin{array}{l}-0.15 \\
(0.23)\end{array}$ & $\begin{array}{c}-0.17 \\
(0.23)\end{array}$ & $\begin{array}{l}0.032 \\
(0.21)\end{array}$ \\
\hline Same legal system $_{i j}$ & $\begin{array}{l}0.54^{* \star *} \\
(0.088)\end{array}$ & $\begin{array}{l}0.54^{* * *} \\
(0.089)\end{array}$ & $\begin{array}{l}0.49^{* * *} \\
(0.088)\end{array}$ & $\begin{array}{l}0.44^{* * *} \\
(0.084)\end{array}$ & $\begin{array}{l}0.39^{\star * *} \\
(0.080)\end{array}$ & $\begin{array}{l}0.71^{* * *} \\
(0.11)\end{array}$ & $\begin{array}{c}0.74^{* * *} \\
(0.12)\end{array}$ & $\begin{array}{c}0.72^{\star * *} \\
(0.11)\end{array}$ & $\begin{array}{l}0.72^{\star * *} \\
(0.11)\end{array}$ & $\begin{array}{l}0.72^{\star * \star} \\
(0.095)\end{array}$ \\
\hline Common language $_{i j}$ & $\begin{array}{c}0.14 \\
(0.14)\end{array}$ & $\begin{array}{c}0.11 \\
(0.14)\end{array}$ & $\begin{array}{l}0.34^{\star *} \\
(0.14)\end{array}$ & $\begin{array}{c}0.44^{\star * *} \\
(0.13)\end{array}$ & $\begin{array}{c}0.15 \\
(0.12)\end{array}$ & $\begin{array}{c}0.59^{\star * *} \\
(0.20)\end{array}$ & $\begin{array}{c}0.58^{\star \star *} \\
(0.20)\end{array}$ & $\begin{array}{c}0.74^{\star \star *} \\
(0.21)\end{array}$ & $\begin{array}{c}0.82^{\star \star \star} \\
(0.21)\end{array}$ & $\begin{array}{c}0.51^{\star * *} \\
(0.18)\end{array}$ \\
\hline $\ln \left(\right.$ Remoteness $\left._{\mathrm{it}}\right)$ & $\begin{array}{l}-0.87^{*} \\
(0.50)\end{array}$ & $\begin{array}{c}-0.59 \\
(0.54)\end{array}$ & $\begin{array}{l}-0.97 \\
(0.98)\end{array}$ & $\begin{array}{c}-1.46 \\
(0.92)\end{array}$ & $\begin{array}{c}0.10 \\
(0.51)\end{array}$ & $\begin{array}{c}-0.34 \\
(0.87)\end{array}$ & $\begin{array}{l}-1.22 \\
(0.83)\end{array}$ & $\begin{array}{c}0.34 \\
(1.02)\end{array}$ & $\begin{array}{l}0.098 \\
(1.04)\end{array}$ & $\begin{array}{l}-0.40 \\
(0.83)\end{array}$ \\
\hline $\ln \left(\right.$ Remoteness $\left._{\mathrm{jt}}\right)$ & $\begin{array}{l}-0.34 \\
(0.48)\end{array}$ & $\begin{array}{r}-0.021 \\
(0.58)\end{array}$ & $\begin{array}{l}-1.09 \\
(1.01)\end{array}$ & $\begin{array}{c}-2.00^{\star *} \\
(0.86)\end{array}$ & $\begin{array}{c}-0.44 \\
(0.73)\end{array}$ & $\begin{array}{c}0.59 \\
(0.97)\end{array}$ & $\begin{array}{c}0.37 \\
(1.05)\end{array}$ & $\begin{array}{c}0.94 \\
(1.09)\end{array}$ & $\begin{array}{c}0.55 \\
(1.09)\end{array}$ & $\begin{array}{c}0.23 \\
(0.91)\end{array}$ \\
\hline $\ln \left(\right.$ Eff. exch. rate $\left.{ }_{i t}\right)$ & $\begin{array}{c}-0.21 \\
(0.16)\end{array}$ & $\begin{array}{l}0.56^{\star *} \\
(0.22)\end{array}$ & $\begin{array}{l}-0.34 \\
(0.21)\end{array}$ & $\begin{array}{l}-0.19 \\
(0.21)\end{array}$ & $\begin{array}{c}0.23 \\
(0.19)\end{array}$ & $\begin{array}{c}-0.57^{* * *} \\
(0.20)\end{array}$ & $\begin{array}{l}-0.43^{*} \\
(0.25)\end{array}$ & $\begin{array}{c}-0.55^{\star * *} \\
(0.21)\end{array}$ & $\begin{array}{c}-0.64^{\star \star *} \\
(0.20)\end{array}$ & $\begin{array}{c}-0.52^{\star \star \star} \\
(0.18)\end{array}$ \\
\hline $\ln \left(\right.$ Eff. exch. rate $\left._{j t}\right)$ & $\begin{array}{l}-0.13 \\
(0.21)\end{array}$ & $\begin{array}{c}0.14 \\
(0.30)\end{array}$ & $\begin{array}{c}-0.57^{* *} \\
(0.28)\end{array}$ & $\begin{array}{l}-0.39 \\
(0.25)\end{array}$ & $\begin{array}{l}-0.17 \\
(0.23)\end{array}$ & $\begin{array}{c}-0.034 \\
(0.20)\end{array}$ & $\begin{array}{c}0.13 \\
(0.22)\end{array}$ & $\begin{array}{l}0.027 \\
(0.21)\end{array}$ & $\begin{array}{l}0.030 \\
(0.21)\end{array}$ & $\begin{array}{c}-0.094 \\
(0.18)\end{array}$ \\
\hline $\mathrm{EPL}_{\text {it }}$ & $\begin{array}{c}-0.26^{\star *} \\
(0.11)\end{array}$ & $\begin{array}{c}-0.54^{\star \star *} \\
(0.20)\end{array}$ & $\begin{array}{l}-0.15 \\
(0.12)\end{array}$ & $\begin{array}{l}-0.12 \\
(0.11)\end{array}$ & $\begin{array}{l}-0.20^{*} \\
(0.11)\end{array}$ & $\begin{array}{c}-0.45^{\star \star *} \\
(0.14)\end{array}$ & $\begin{array}{c}-0.63^{\star * *} \\
(0.21)\end{array}$ & $\begin{array}{c}-0.27^{* *} \\
(0.13)\end{array}$ & $\begin{array}{c}-0.27^{* *} \\
(0.13)\end{array}$ & $\begin{array}{c}-0.45^{\star \star *} \\
(0.13)\end{array}$ \\
\hline $\mathrm{EPL}_{\mathrm{jt}}$ & $\begin{array}{c}-0.58^{\star \star *} \\
(0.16)\end{array}$ & $\begin{array}{c}-0.59^{* * *} \\
(0.21)\end{array}$ & $\begin{array}{c}-0.67^{* \star *} \\
(0.18)\end{array}$ & $\begin{array}{c}-0.62^{\star * *} \\
(0.17)\end{array}$ & $\begin{array}{c}-0.51^{\star * *} \\
(0.19)\end{array}$ & $\begin{array}{l}-0.11 \\
(0.17)\end{array}$ & $\begin{array}{l}-0.51^{*} \\
(0.30)\end{array}$ & $\begin{array}{c}-0.011 \\
(0.17)\end{array}$ & $\begin{array}{l}0.058 \\
(0.17)\end{array}$ & $\begin{array}{l}-0.16 \\
(0.16)\end{array}$ \\
\hline $\mathrm{EEA}_{\mathrm{it}}$ & $\begin{array}{c}0.23 \\
(0.17)\end{array}$ & $\begin{array}{c}0.21 \\
(0.17)\end{array}$ & $\begin{array}{c}0.24 \\
(0.21)\end{array}$ & $\begin{array}{l}-0.18 \\
(0.22)\end{array}$ & $\begin{array}{c}0.25 \\
(0.15)\end{array}$ & $\begin{array}{c}0.51^{\star * *} \\
(0.18)\end{array}$ & $\begin{array}{c}0.50^{* * *} \\
(0.16)\end{array}$ & $\begin{array}{l}0.36^{\star} \\
(0.19)\end{array}$ & $\begin{array}{l}0.31^{*} \\
(0.19)\end{array}$ & $\begin{array}{l}0.38^{* *} \\
(0.15)\end{array}$ \\
\hline $\mathrm{EEA}_{\mathrm{jt}}$ & $\begin{array}{c}0.81^{\star * *} \\
(0.27)\end{array}$ & $\begin{array}{c}0.44^{*} \\
(0.26)\end{array}$ & $\begin{array}{c}0.86^{\star * *} \\
(0.30)\end{array}$ & $\begin{array}{c}0.37 \\
(0.28)\end{array}$ & $\begin{array}{l}0.67^{\star *} \\
(0.28)\end{array}$ & $\begin{array}{l}0.031 \\
(0.25)\end{array}$ & $\begin{array}{l}-0.11 \\
(0.23)\end{array}$ & $\begin{array}{c}-0.087 \\
(0.26)\end{array}$ & $\begin{array}{l}-0.17 \\
(0.26)\end{array}$ & $\begin{array}{c}0.43^{*} \\
(0.22)\end{array}$ \\
\hline $\mathrm{EEA}_{\mathrm{ijt}}$ & $\begin{array}{c}0.35 \\
(0.25)\end{array}$ & $\begin{array}{c}0.37 \\
(0.26)\end{array}$ & $\begin{array}{l}0.047 \\
(0.26)\end{array}$ & $\begin{array}{c}0.23 \\
(0.25)\end{array}$ & $\begin{array}{c}0.23 \\
(0.21)\end{array}$ & $\begin{array}{c}0.22 \\
(0.18)\end{array}$ & $\begin{array}{c}0.14 \\
(0.18)\end{array}$ & $\begin{array}{c}0.13 \\
(0.19)\end{array}$ & $\begin{array}{c}0.14 \\
(0.19)\end{array}$ & $\begin{array}{c}0.19 \\
(0.17)\end{array}$ \\
\hline $\mathrm{EA}_{\mathrm{it}}$ & $\begin{array}{l}-0.038 \\
(0.099)\end{array}$ & $\begin{array}{l}-0.012 \\
(0.097)\end{array}$ & $\begin{array}{l}-0.23^{*} \\
(0.12)\end{array}$ & $\begin{array}{l}-0.22^{*} \\
(0.11)\end{array}$ & $\begin{array}{c}-0.0087 \\
(0.11)\end{array}$ & $\begin{array}{c}-0.058 \\
(0.11)\end{array}$ & $\begin{array}{l}-0.17 \\
(0.13)\end{array}$ & $\begin{array}{l}-0.22^{*} \\
(0.13)\end{array}$ & $\begin{array}{l}-0.28^{\star *} \\
(0.13)\end{array}$ & $\begin{array}{l}0.033 \\
(0.11)\end{array}$ \\
\hline $\mathrm{EA}_{\mathrm{jt}}$ & $\begin{array}{l}0.39 * * * \\
(0.097)\end{array}$ & $\begin{array}{l}0.37^{\star \star \star} \\
(0.096)\end{array}$ & $\begin{array}{c}0.17 \\
(0.11)\end{array}$ & $\begin{array}{l}0.21^{\star *} \\
(0.10)\end{array}$ & $\begin{array}{l}0.40^{\star * *} \\
(0.10)\end{array}$ & $\begin{array}{l}0.48^{\star \star *} \\
(0.13)\end{array}$ & $\begin{array}{l}0.53^{\star \star *} \\
(0.13)\end{array}$ & $\begin{array}{l}0.34^{\star *} \\
(0.14)\end{array}$ & $\begin{array}{l}0.29^{* *} \\
(0.14)\end{array}$ & $\begin{array}{l}0.49^{\star * *} \\
(0.12)\end{array}$ \\
\hline $\mathrm{EA}_{\mathrm{ijt}}$ & $\begin{array}{l}0.28^{*} \\
(0.16)\end{array}$ & $\begin{array}{c}0.22 \\
(0.16)\end{array}$ & $\begin{array}{l}0.28^{\star} \\
(0.15)\end{array}$ & $\begin{array}{c}0.22 \\
(0.14)\end{array}$ & $\begin{array}{c}0.13 \\
(0.15)\end{array}$ & $\begin{array}{c}0.23 \\
(0.16)\end{array}$ & $\begin{array}{c}0.21 \\
(0.17)\end{array}$ & $\begin{array}{c}0.23 \\
(0.17)\end{array}$ & $\begin{array}{c}0.27 \\
(0.17)\end{array}$ & $\begin{array}{l}0.022 \\
(0.15)\end{array}$ \\
\hline NAFTA $_{i j t}$ & $\begin{array}{l}-0.77^{* *} \\
(0.30)\end{array}$ & $\begin{array}{l}-0.87^{* * *} \\
(0.33)\end{array}$ & $\begin{array}{l}-1.02^{* \star \star} \\
(0.29)\end{array}$ & $\begin{array}{l}-0.73^{\star * \star} \\
(0.25)\end{array}$ & $\begin{array}{l}-0.50^{\star *} \\
(0.25)\end{array}$ & $\begin{array}{l}-0.56 \\
(0.54)\end{array}$ & $\begin{array}{l}-0.28 \\
(0.63)\end{array}$ & $\begin{array}{l}-0.044 \\
(0.47)\end{array}$ & $\begin{array}{l}-0.25 \\
(0.49)\end{array}$ & $\begin{array}{l}-0.48 \\
(0.53)\end{array}$ \\
\hline ETCR $_{\text {it }}$ & & & $\begin{array}{l}-0.15^{\star} \\
(0.078)\end{array}$ & $\begin{array}{c}-0.12 \\
(0.074)\end{array}$ & & & & $\begin{array}{l}-0.30 * * * \\
(0.085)\end{array}$ & $\begin{array}{l}-0.36^{* * *} \\
(0.085)\end{array}$ & \\
\hline $\mathrm{ETCR}_{\mathrm{jt}}$ & & & $\begin{array}{l}-0.18^{\star *} \\
(0.080)\end{array}$ & $\begin{array}{l}-0.13^{\star} \\
(0.074)\end{array}$ & & & & $\begin{array}{l}-0.25^{\star *} \\
(0.10)\end{array}$ & $\begin{array}{l}-0.25^{\star \star} \\
(0.10)\end{array}$ & \\
\hline $\mathrm{N}$ & 9634 & 6036 & 8917 & 8761 & 11584 & 9634 & 6036 & 8917 & 8761 & 11584 \\
\hline $\mathrm{R}^{2}$ & 0.917 & 0.920 & 0.831 & 0.854 & 0.806 & 0.821 & 0.835 & 0.807 & 0.810 & 0.826 \\
\hline $\begin{array}{l}\text { End of sample } \\
\text { Sample }\end{array}$ & $\begin{array}{c}2013 \\
\text { OECD31 }\end{array}$ & $\begin{array}{c}2008 \\
\text { OECD31 }\end{array}$ & $\begin{array}{c}2013 \\
\text { OECD31 }\end{array}$ & $\begin{array}{c}2013 \\
\text { OECD31 }\end{array}$ & $\begin{array}{c}2013 \\
\text { OECD34 }\end{array}$ & $\begin{array}{c}2013 \\
\text { OECD31 }\end{array}$ & $\begin{array}{c}2008 \\
\text { OECD31 }\end{array}$ & $\begin{array}{c}2013 \\
\text { OECD31 }\end{array}$ & $\begin{array}{c}2013 \\
\text { OECD31 }\end{array}$ & $\begin{array}{c}2013 \\
\text { OECD34 }\end{array}$ \\
\hline $\begin{array}{l}\text { Country and year } \\
\text { fixed effects }\end{array}$ & YES & YES & YES & YES & YES & YES & YES & YES & YES & YES \\
\hline $\begin{array}{l}\text { Controls for size and } \\
\text { factor dissimilarities }\end{array}$ & NO & NO & $\mathrm{NO}$ & YES & NO & NO & NO & NO & YES & NO \\
\hline
\end{tabular}

Note: Asterisks $\left({ }^{*},{ }^{* *},{ }^{* * *}\right)$ indicate the significance level $(10 \%, 5 \%, 1 \%)$ of the coefficients. i denotes the inward country and $\mathrm{j}$ the outward country. Standard errors adjusted for country-pair clusters are in parentheses. $Y_{i t}=$ nominal GDP in US Dollars, Dist ${ }_{i j}=$

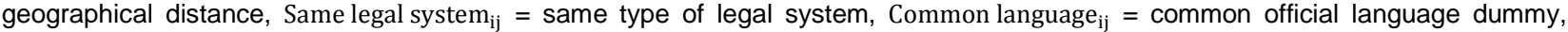
Eff. exchange rate ${ }_{i t}=$ Nominal effective exchange rate, $E E A_{i t}=$ European Economic Area dummy, $E A_{i j t}=$ euro area pair dummy, NAFTA $_{i j t}=$ North American Free Trade Agreement pair dummy, $\mathrm{EPL}_{\mathrm{it}}=$ Strictness of employment protection for regular contracts, $E T C R_{\mathrm{it}}=$ Electricity, Transport and Communication Regulation indicator. Poisson = Poisson pseudo-maximum likelihood. 